\title{
Nociceptor neurons impair cancer immunosurveillance
}

Sébastien Talbot ( $\square$ sebastien.talbot@umontreal.ca )

Université de Montréal

Mohammad Balood

Universite de Montreal

Maryam Ahmadi

Universite de Montreal

Tuany Eichwald

Universite de Montreal

Abdelilah Majdoubi

Universite de Montreal

Hannah Merrison

Boston Children's Hospital

Alexandre Parrin

Boston Children's Hospital

Benjamin Doyle

Boston Children's Hospital

Chris Lucido

Sanford Research

Daniel Vermeer

Sanford Research

Siyi Huang

Cygnal Therapeutics

Lexiang Ji

Cygnal Therapeutics

Hongyue Dai

Cygnal Therapeutics

Chengyi Shu

Cygnal Therapeutics

Katiane Roversi

Universite de Montreal

Sini Thomas

Universite de Montreal

Corey Seehus 
Harvard Medical School

\section{Simmie Foster}

Massachusetts General Hospital

\section{Ali Ahmadi}

Universite de Montreal

\section{Moutih Rafei}

Universite de Montreal

\section{Manu Rangachari}

Université Laval, Québec QC Canada

Jacques Thibodeau

Université de Montréal

Nader Ghasemlou

Queen\&\#39;s University

\section{Sonia del Rincon}

McGill University

\section{Ronny Drapkin}

University of Pennsylvania https://orcid.org/0000-0002-6912-6977

\section{Paola Vermeer}

Sanford Research https://orcid.org/0000-0003-2370-8223

\section{Clifford Woolf}

F.M. Kirby Neurobiology Research Center, Boston Children's Hospital https://orcid.org/0000-0002-66363897

\section{Biological Sciences - Article}

Keywords: Nociceptor neurons, neuro-immunology, tumor innervation, melanoma, exhaustion, cytotoxic CD8+ T-cells, CGRP, TRPV1, QX-314, Botox.

Posted Date: June 9th, 2021

DOI: https://doi.org/10.21203/rs.3.rs-580730/v1

License: (c) (1) This work is licensed under a Creative Commons Attribution 4.0 International License. Read Full License

Version of Record: A version of this preprint was published at Nature on November 2nd, 2022. See the published version at https://doi.org/10.1038/s41586-022-05374-w. 
Mohammad Balood 1,2, ${ }^{4,5}$, Benjamin Doyle ${ }^{4,5}$, Chris T. Lucido ${ }^{6}$, Daniel W. Vermeer ${ }^{6}$, Siyi Huang ${ }^{7}$, Lexiang Ji ${ }^{7}$, Hongyue Dai ${ }^{7}$, Chengyi J. Shu ${ }^{7}$, Katiane Roversi ${ }^{1}$, Sini C. Thomas ${ }^{1}$, Corey R. Seehus ${ }^{4,5}$, Simmie L. Foster ${ }^{8}$, Ali Ahmadi ${ }^{1}$, Moutih Rafei ${ }^{1}$, Manu Rangachari ${ }^{2}$, Jacques Thibodeau ${ }^{3}$, Nader Ghasemlou $4,5,11$, Sonia Del Rincon ${ }^{9}$, Ronny Drapkin ${ }^{10}$, Paola D. Vermeer ${ }^{6}$, Clifford J. Woolf 4,5 , and Sebastien Talbot ${ }^{1, \#}$

1 Département de Pharmacologie et Physiologie, Université de Montréal, Montréal, Canada

2 Département de Médecine Moléculaire, Faculté de Médecine, Université Laval, Québec, Canada

${ }^{3}$ Département de microbiologie, infectiologie et immunologie, Université de Montréal, Montréal, Canada

${ }^{4}$ F.M. Kirby Neurobiology Center, Children's Hospital Boston, Boston, USA

${ }^{5}$ Department of Neurobiology, Harvard Medical School, Boston, USA

${ }^{6}$ Sanford Research, Cancer Biology and Immunotherapy Sioux Falls, USA.

${ }^{7}$ Cygnal Therapeutics, Cambridge, USA

${ }^{8}$ Depression Clinical Research Program, Massachusetts General Hospital, Boston, USA

${ }^{9}$ Department of Oncology, McGill University, Montréal, Canada

10 Penn Ovarian Cancer Research Center, University of Pennsylvania, Perelman School of Medicine, Philadelphia, USA.

11 Department of Anesthesiology and perioperative medicine, Queen's University. Kingston, Canada.

${ }^{*}$ Denotes equal contribution

Running title: Neurons decrease cancer immunosurveillance

\# Lead Contact:

Sebastien Talbot, PhD

Phone: 514-343-6111. Ext: 32787

Email: sebastien.talbot@umontreal.ca

Keywords. Nociceptor neurons, neuro-immunology, tumor innervation, melanoma, exhaustion, cytotoxic CD8 ${ }^{+} \mathrm{T}_{\text {-cells, }}$ CGRP, TRPV1, QX-314, Botox.

Summary. Solid tumors are innervated by nerve fibers that arise from the autonomic and sensory peripheral nervous systems. In prostate cancer, doublecortin-expressing neural progenitors initiate autonomic adrenergic neurogenesis ${ }^{1}$ which facilitates tumor development and dissemination ${ }^{2}$, via an angiogenic switch that fuels cancer growth ${ }^{3,4}$. Similarly, a loss of TP53 drives the reprogramming of tumor-innervating sensory nerves into adrenergic neurons in head and neck tumors, which promotes tumor growth ${ }^{5}$. However, the impact of tumor neo-innervation by pain-initiating sensory neurons remains unclear. We show that melanoma cells interact with nociceptors, increasing neurite outgrowth, responsiveness to noxious ligands, and neuropeptide release. In turn, CGRP, a nociceptor-produced neuropeptide, directly increases exhaustion of cytotoxic CD8 ${ }^{+} \mathrm{T}$ cells (PD1+ ${ }^{+}$ag $3^{+} \mathrm{Tim}^{+}{ }^{+} \mathrm{FNY} \mathrm{F}^{-}$), limiting their capacity to eliminate melanoma. Genetic Nav1.8 or TRPV1 lineage ablation, local pharmacological silencing or blockade of neuropeptide release from tumor-innervating nociceptors, and the antagonism of the CGRP receptor RAMP1, all blunt tumor-infiltrating leukocyte exhaustion, and tumor growth, nearly tripling survival of B16F10inoculated mice. Inversely, $\mathrm{CD}^{+}{ }^{+}$T-cell exhaustion increased following optogenetic activation of tumor-innervating Nav1.8 neurons $^{+}$and was rescued in sensory neuron depleted mice treated with recombinant CGRP. In comparison to wild-type CD8 ${ }^{+}$ T-cells, RAMP1-1- CD8 ${ }^{+}$T-cells were protected from undergoing exhaustion when co-transplanted into tumor-bearing Rag1 deficient mice. Single-cell RNA sequencing of patient tumors revealed that intratumoral RAMP1-expressing CD8 ${ }^{+} \mathrm{T}_{\text {-cells }}$ are more exhausted than their RAMP1 negative counterparts. RAMP1 expression in intratumoral CD8 ${ }^{+}$T-cells was also associated with resistance to immune checkpoint inhibitor treatment, while RAMP1 overexpression within the tumor correlated with a worse clinical prognosis. We conclude that reducing CGRP release from tumor-innervating nociceptors, by eliminating its immunomodulatory action on cytotoxic CD8 ${ }^{+}$T-cells, constitutes a useful strategy to safeguard anti-tumor immunity.

Main. Cytotoxic T-cells express a variety of receptors, including PD-1 (Programmed cell Death protein-1), Lag-3 (Lymphocyte Activation Gene-3) and Tim-3 ( $T$-cell immunoglobulin and mucin domain-containing protein 3$)^{6-9}$, which inhibit T-cell function after being activated by their cognate ligands. These checkpoint receptors ensure that immune responses to damage or infection are kept in check, preventing overly intense responses that might damage healthy cells ${ }^{10}$. Tumor cells express ligands for these immune checkpoints, which, when activated, block the cytolytic functions of T-cells, favoring cancer cell survival ${ }^{10-12}$. That neo-innervation is a prominent feature in cancer, together with the diverse actions of neuropeptides on immune cells ${ }^{13-23}$ led us to explore whether local release of neuropeptides from activated nociceptors may favor cancer growth by suppressing immune surveillance.

Breast cancers show a hyper-sympathetic innervation but a decreased parasympathetic nerve density ${ }^{15}$, whereas prostate cancers are infiltrated with cholinergic fibers and are surrounded by adrenergic fibers ${ }^{2}$. We probed the extent of melanoma 
innervation by nociceptor by testing for the presence of TRPV1+ (Transient Receptor Potential cation channel subfamily Vanilloid 1) nociceptor neurons in melanoma patients' biopsies (Supp. Fig. 1). TRPV1 immunolabelling was increased $\sim 2$-fold in the tumor compared to adjacent healthy tissue, a feature observed in each of the ten-biopsy tested. Intra-tumoral TIL (TumorInfiltrating Leukocytes) numbers correlated with increased TRPV1 immunolabelling $\left(R^{2}=0.63 ; p<0.05\right.$; retrospective correlation analysis performed on patient's pathology report). These data indicate that patients' melanomas are densely innervated by sensory neurons and they may impact intra-tumoral cell numbers.

To explore this, we inoculated a GFP-expressing melanoma (B16F10-eGFP) cell line into Nav1.8cre::Td-tomatof f/wt mice and used iDISCO as a mean to obtain a 3-dimensional representation of the tumor innervation ${ }^{24,25}$. Twenty-two days after implantation, we found abundant Nav1. $8^{+}$nociceptor neurons around and within the tumor (Fig. 1A). Next, we inoculated (i.d.; $1 \times 10^{5}$ ) either B16F10 cells or non-cancerous keratinocytes (MPEK-BL6) into 8-week-old male and female nociceptor neurons reporter mice (TRPV1 cre::Td-tomatof/wt) ${ }^{26}$. Fourteen days post-inoculation, only the B16F10 tumor cells induced significant TRPV1+ nociceptor axonal innervation and outgrowth, near the injected cells (Supp. Fig. 2).

When co-cultured with B16F10-eGFP melanoma cells, fluorescently labeled (TRPV1 cre:::Td-tomato $^{\mathrm{f} / \text { wt }}$ ) DRG nociceptor neurites largely extend toward the B16F10 cells, often forming physical contacts (Fig. 1B). The average neurite length of nociceptor neurons increased (number of intersecting radii; Fig. 1C-D) while the overall neuronal arborization decreased (ramification index; Fig. 1E) when co-cultured with B16F10-eGFP cells. In addition, cultured L3-L5 DRG nociceptors harvested from tumorinoculated mice (on d14) extend longer neurites than their counterparts harvested from keratinocyte-injected mice (not shown). These findings indicate that nociceptors' neurite outgrowth is enhanced when in proximity to melanoma cells.

Given that melanoma promotes axonogenesis leading to tumor innervation (Fig. 1A; Supp. Fig. 1-2), we next aimed to examine whether such physical proximity modulates the sensitivity of the nociceptor, which detect signals from their local environment. To test this, we measured changes in calcium flux in nociceptors induced in response to sub-threshold concentrations of capsaicin (an agonist of the heat sensing channel TRPV1), mustard oil (an agonist of the chemical sensing channel TRPA1), and ATP (an agonist of the proton-sensing channel P2X3R). When nociceptors are cultured without melanoma cells, few responded to the ligands at the concentrations selected. However, the number of responsive neurons increased when cocultured with B16F0 melanoma cells, an effect made more robust when exposed to the highly metastatic melanoma cell line B16F10 (Fig. 1F). Similarly, the amplitude of calcium flux responses to the ligands was greater in ipsilateral L3-L5 DRG neurons harvested from fourteen-days tumor-inoculated mice as compared to those harvested from non-tumorigenic keratinocyteinjected mice (Fig. 1G). Signals from the melanoma heighten nociceptor sensitivity.

We next set out to identify the mechanism through which melanoma sensitizes nociceptor neurons. To best mimic the interactions at play in the melanoma microenvironment, Tc1-stimulated OVA-specific cytotoxic CD8 ${ }^{+}$T-cells (OT-1 mice), naïve

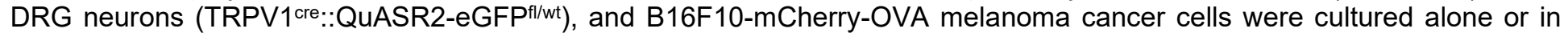
combination. After 48h, the cells were harvested, FACS-purified and RNA sequenced. Differentially expressed genes (DEG) were calculated between the ten tested groups (Supp. Fig. 3A-B). Among others, we found that Slpi (Secretory Leukocyte Protease Inhibitor) was overexpressed in cancer cells when co-cultured with DRG neurons ( 3.6-fold) or OVA-specific cytotoxic CD8 ${ }^{+}$T-cells ( 270-fold), and when exposed to both populations ( 150 fold; Supp. Fig. 3A-B). Inversely, Slpi levels were not impacted in OVA-specific cytotoxic CD8 ${ }^{+}$T-cells or DRG neurons when cultured alone or with B16F10-mCherry-OVA (Supp. Fig. 3B). B16F10-mCherry-OVA, when co-cultured with naïve DRG neurons and OVA-specific cytotoxic CD8 ${ }^{+}$T-cell, increased SLPI secretion into the culture medium, an effect maximal after 48h ( 200 -fold; Supp. Fig. 3C). Of note, the melanomasecreted and pain-inducing cytokines TSLP and HMGB1 were not detected (data not shown), while OVA-specific cytotoxic CD8 ${ }^{+}$T-cell only secreted minimal levels of SLPI (Supp. Fig. 3C).

SLPI protects epithelial cells from serine protease activity, enhances regeneration of transected retinal ganglion cell axons ${ }^{27}$ and neural stem cell proliferation ${ }^{28}$. While these data show that SLPI can act on neurons, its role in nociception remains untested. To address this, we measured whether SLPI directly activates cultured DRG neurons using calcium microscopy. We found that SLPI $(10-10000 \mathrm{ng} / \mathrm{ml}$ ) activates $\sim 20 \%$ of DRG neurons (Supp. Fig. 3D-F), including $\sim 42 \%(n=261 / \mathrm{n}=614)$ of capsaicin-responsive neurons (Supp. Fig. 3E). SLPI-responsive neurons are mostly small-medium-sized neurons (Supp. Fig. 3G-H; mean area $=151 \mu \mathrm{m}^{2}$ ) and $\sim 90 \%$ of them are capsaicin responsive (Supp. Fig. 3I), compatible with them being nociceptors. Next, the right hindpaw of naïve mice were injected with saline or SLPI (1 $\mu$; $25 \mu \mathrm{L}$; i.d.) and the impact of this was measured $(0-6 h)$ on the mouse thermal nociceptive threshold. In comparison to the contralateral paw, the ipsilateral paw injected with SLPI showed thermal hypersensitivity. Saline had no impact on the mice thermal sensitivity threshold (Supp. Fig. 3J). We next sought to test, if such neuronal hypersensitivity would lead to the release of immunomodulatory neuropeptides. As opposed to B16F10 cells alone, DRG neurons co-cultured with B16F10 cells $\left(5 \times 10^{4}\right.$ cells, $\left.24 \mathrm{~h}\right)$ actively release CGRP in the media (Fig. 1H). Similarly, recombinant SLPI, at a level corresponding to the one produced by melanoma cells (100-1000 $\mathrm{pg} / \mathrm{ml}$ ), prompted CGRP release from cultured naïve DRG neurons (Supp. Fig. 3K). Melanoma-secreted SLPI acts on nociceptors to trigger calcium influx, neuropeptide release and thermal hypersensitivity indicating that the sensory neurons detect the presence of the cancer cells and react to this. 
These data prompted us to test whether melanoma alter the nociceptor neurons transcriptome. To do this, naïve DRG neurons (TRPV1 cre::QuASR2-eGFPflwt) were cultured alone or in combination with B16F10-mCherry-OVA. After 48h, TRPV1+ nociceptors were harvested, FACS-purified and RNA-sequenced. Differentially expressed genes (DEG) were calculated and the neuropeptide gene Calca (encoding for CGRP) as well as the NGF receptor Trka were found to be overexpressed in cancerexposed nociceptors (Supp. Fig. 4). TRKA overexpression may help drive melanoma-induced pain hypersensitivity, while CGRP (encoded by Calca) may, when released from activated nociceptors, immunomodulate TILs.

Cytotoxic CD8 ${ }^{+}$T-cells express multiple neuropeptide receptors ( $(210)$, including the CGRP receptor RAMP1 (Supp. Fig. 5A). Given that nociceptors readily interact with $\mathrm{CD} 8^{+} \mathrm{T}$-cells in culture and that the neuropeptides they release (Supp. Fig. 5B) block $T_{H} 1$ immunity ${ }^{29-32}$, we aimed to test whether these mediators could drive $\mathrm{CD}^{+}$T-cell expression of immune checkpoint receptors. To do this, splenocyte-isolated $\mathrm{CD} 8^{+} \mathrm{T}$-cells were cultured under type $1 \mathrm{CD} 8^{+} \mathrm{T}$-cell-stimulating conditions for two days and subsequently co-cultured with DRG neurons for an additional four days. We found that neuron stimulation with capsaicin increased the proportion of PD1 ${ }^{+} \mathrm{Lag}_{3}{ }^{+} \mathrm{Tim} 3^{+}$expressing $\mathrm{CD} 8^{+} \mathrm{T}$ cells while it decreased the levels of IFNy ${ }^{+}, \mathrm{TNFa}^{+}$, IL2+ (Fig. 2A-B; Supp. Fig. 6; measured after four days co-culture). These effects were absent when CD8 ${ }^{+}$T-cells were cocultured in absence of TRPV1 ${ }^{+}$neurons (TRPV1 ${ }^{\text {cre }}:$ DTA ${ }^{\text {fl/wt; }}$; Fig. 2A-B; Supp. Fig. 6). Capsaicin had no measurable impact on $\mathrm{CD}^{+} \mathrm{T}$-cells in the absence of DRG neurons (Fig. 2A-B; Supp. Fig. 6). These data suggest that when activated peptidergic neurons drive the expression of $\mathrm{CD} 8^{+} \mathrm{T}$-cell exhaustion markers in co-culture.

To further test this, Tc1-stimulated cytotoxic $C D 8^{+}$T-cells were exposed to fresh conditioned media (1:2 dilution) harvested from $\mathrm{KCl}(50 \mathrm{mM})$-stimulated DRG neurons, unstimulated or vehicle exposed neurons. When measured after four days of stimulation, the conditioned media (CM) from KCl- (Fig. 2C-D; Supp. Fig. 7) or capsaicin- (Supp. Fig. 8) activated nociceptor increased the proportion of PD1 $1^{+} \mathrm{Lag}^{+} \mathrm{Tim}^{+}$cytotoxic $\mathrm{CD}^{+} \mathrm{T}$-cells and reduced those which are IFNY ${ }^{+}$and $\mathrm{TNFa}^{+}$. These effects were prevented when cytotoxic CD8 ${ }^{+}$T-cells were co-exposed to the RAMP1 blocker CGRP8-37 $(2 \mu \mathrm{g} / \mathrm{ml})$ or when

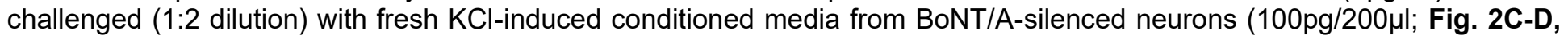
Supp. Fig. 8). These data suggested that nociceptor neuron-released CGRP is responsible for inducing CD8 T-cells exhaustion acting on its cognate receptor RAMP1.

To confirm whether recombinant CGRP has direct immunomodulatory effects on cytotoxic CD8 ${ }^{+}$T-cells we showed that it directly enhanced the proportion of $\mathrm{PD}^{+}{ }^{+} \mathrm{Lag}^{+}{ }^{+} \mathrm{Tim} 3^{+}$cells and decreased the proportion of IFNY ${ }^{+}$and $\mathrm{TNFa}^{+}$cells (Fig. 2E-F, Supp. Fig. 9; measured after four days stimulation). As expected, CGRP had no measurable impact on CD8 T-cells harvested from a RAMP1-- mouse (Fig. 2E-F, Supp. Fig. 9). These data suggest that nociceptor-released CGRP, rather than cell-cell contact, drives the exhaustion of cytotoxic CD8 ${ }^{+}$T-cells.

The co-expression of several exhaustion markers, accompanied by the simultaneous loss of cytotoxic molecules (i.e., IFNy, TNFa) and proliferative capacity (i.e., IL-2) are synonymous with functional exhaustion, as opposed to the expression of one exhaustion marker, which may indicate antigen experienced immune cell activation. Here, we found that PD $1^{+} \mathrm{Lag} 3^{+} \mathrm{Tim}^{+}$ $\mathrm{CD}^{+} \mathrm{T}$-cells reduce their expression of IFNy ${ }^{+}, \mathrm{TNFa}^{+}$, and IL-2 ${ }^{+}$when i) co-cultured with capsaicin-stimulated nociceptor intact neurons (Supp. Fig. 6); ii) stimulated with capsaicin-induced neuron conditioned media (Supp. Fig. 7); or iii) exposed to recombinant CGRP (Supp. Fig. 9). These effects were absent when cytotoxic CD8 ${ }^{+}$T-cells were co-cultured with ablated sensory neurons (Supp. Fig. 6) or in CGRP-exposed RAMP1-- CD8 T-cells (Supp. Fig. 9).

As a litmus test for $\mathrm{CD} 8^{+} \mathrm{T}$-cell exhaustion, we probed whether nociceptor neuron-released neuropeptides blunt the anti-tumor responses of cytotoxic $\mathrm{CD}^{+} \mathrm{T}$-cells though exhaustion of the T-cells. OT1 cytotoxic T-cells induced robust apoptosis of B16F10-mCherry-OVA apoptosis (annexinV ${ }^{+} 7 A A D^{+}$B16F10-mCherry-OVA; Fig. 2G-I). However, B16F10-mCherry-OVA apoptosis was decreased when challenged with i) capsaicin- (Fig. 2G; Supp. Fig. 10; measured after 48h); ii) or KCl- (Fig. 2H; Supp. Fig. 11; measured after 24h) stimulated neuron-derived conditioned media or iii) when directly stimulated with CGRP (Fig. 2I; Supp. Fig. 12; measured after 24h). These effects were absent when OT1 cytotoxic T-cells were exposed to capsaicin-induced conditioned media from sensory neuron ablated mice (Fig. 2G; Supp. Fig. 10); or when treated with $\mathrm{KCl}$ induced conditioned media from intact mice supplemented with a RAMP1 blocker (CGRP $8-37 ; 2 \mu \mathrm{g} / \mathrm{ml}$; Fig. 2H, Supp. Fig. 11). Along with earlier findings that CGRP blocks $\mathrm{CD} 8^{+} \mathrm{T}$-cell proliferation ${ }^{33}$, our data suggest that the crosstalk between nociceptor and CD8 ${ }^{+}$T-cells, via the CGRP-RAMP1 axis, leads to functional exhaustion of CD8 ${ }^{+} \mathrm{T}$-cells, as defined by a reduced capacity to kill melanoma cells (Fig. 2).

The expression of adrenergic and cholinergic nerve fiber markers in tumors is correlated with poor clinical outcomes ${ }^{2}$. Gastric tumor denervation limits growth and vagotomised patients have lower mortality rates associated with intestinal cancer $2,21,34,35$. Nociceptor-produced neuropeptides reduce immunity against bacteria ${ }^{32}$ and fungi ${ }^{36}$, and promote cytotoxic CD $8^{+} \mathrm{T}_{\text {-cells }}$ exhaustion ${ }^{13,14,16,17,19,23}$ (Fig. 2; Supp. Fig. 6-12). We therefore decided to examine the three-way interaction between cancernociceptor-CD8 ${ }^{+}$T-cells using a syngenic mouse model of triple-negative melanoma, an established model of immunosurveillance (10). B16F10-mCherry-OVA cells were inoculated (i.d., $5 \times 10^{5}$ cells) into 8-week-old male and female

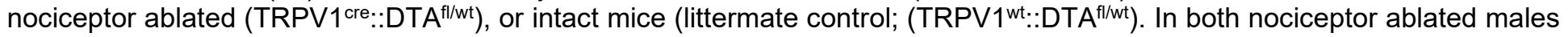
$(n=50)$ and females $(n=68)$, the median length of survival (evaluated until day 21 and determined by reaching a volume of 
$1000 \mathrm{~mm}^{3}$ or other ethical endpoints) increased by 2.5 -fold ( $\mathrm{p} \leq 0.0001 ; 0.4$ Mantel-Haenszel hazard ratio; Fig. 3A). To put these data in context, nociceptor ablation increased the survival rate of B16F10-inoculated mice $\sim 1.5$-fold more than what has been previously observed following aPDL1 blockade (meta-analysis of 16 publications; Supp. Fig. 13I). In another set of mice analyzed fifteen days post-tumor inoculation, we found that tumor growth ( 3.5-fold; Fig 3B; Supp. Fig. 13A-F), weight (2.4fold; Supp. Fig. 13G) and size (Supp. Fig. 13H) were reduced in mice lacking nociceptors. Overall, the presence of nociceptors in melanoma increases cancer growth.

Tumor-specific sympathetic denervation downregulates the expression of PDL1, PD1, and FOXP3 in tumor-infiltrating lymphocytes (TIL), whereas parasympathetic innervation decreases the expression of PD1 and PDL1. Immunocytes exhaustion correlates with relative distance from sympathetic terminals ${ }^{15}$. Aligned with these data, nociceptor-released CGRP increases the exhaustion of cultured cytotoxic CD8 T cells and reduces their elimination of B16F10 cells (Fig. 2). Given this finding, we next tested if nociceptor ablation impacts CD8 T-cells exhaustion in vivo. Fifteen days post B16F10 inoculation (volume of $\sim 800 \mathrm{~mm}^{3}$ in littermate control mice), we found that the genetic ablation of nociceptors increased the number of total (Fig. 3C) and cytotoxic (IFNy+, Fig. 3D; TNFa ${ }^{+}$, Supp. Fig. 14; IL-2, Supp. Fig. 14) tumor-infiltrating CD8 ${ }^{+}$T-cells. The ablation of nociceptors also reduced the proportion of exhausted $\left(\mathrm{PD} 1^{+} \mathrm{Lag}^{+} \mathrm{Tim}^{+}\right)$tumor-infiltrating $\mathrm{CD} 8^{+} \mathrm{T}$-cells (Fig. 3E; Supp. Fig. 14). Similar effects were observed in tumor-draining lymph node CD8+ T-cells (Supp. Fig. 15).

As measured fifteen days post-B16F10-OVA inoculation, we found that on producing a systemic depletion of CD3 ${ }^{+} \mathrm{T}_{\text {-cells }}$ using aCD3 (Supp. Fig. 16A) there were no difference in tumor growth (Fig. 3F) and weight (Supp. Fig. 16B) between nociceptor intact and ablated mice. These data imply that the slower tumor growth found in nociceptor ablated mice depends on nociceptor-induced lymphocyte exhaustion. To support this conclusion, we next compared the impact of nociceptors on the growth of an immunogenic and a non-immunogenic isogenic melanoma model. YUMMER1.7 is a highly immunogenic derivative of the Braf ${ }^{\vee 600 E} \mathrm{Cdkn} 2 \mathrm{a}^{-1-} \mathrm{Pten}^{-/}$cell line that has been modified by UV exposure, and represents a clinically relevant melanoma model ${ }^{37}$. Twelve days post-YUMMER1.7 inoculation, we found that the genetic ablation of nociceptor neurons decreased tumor growth ( 3.3 fold; Fig. 3G), size (Supp. Fig. 17A) and weights ( 3.1 fold; Supp. Fig. 17B). As in the case of B16F10-OVA, ablation of nociceptor increased the intra-tumoral number of total ( $\sim 7.2$-fold), IFNy $\left(\sim 9.1\right.$-fold), and TNFa ${ }^{+}(\sim 9$ fold) $\mathrm{CD} 8^{+} \mathrm{T}$-cells and concomitantly reduced their exhaustion ( 3.6 -fold; Supp. Fig. 17C-F). In contrast, YUMM1.7 is the parental and non-immunogenic ${ }^{38}$ counterpart of YUMMER1.7. Fourteen days post YUMM1.7 inoculation we observed that the absence of nociceptor neurons did not impact either tumor growth rate or TILs exhaustion (Supp. Fig. 18). We next sought to further test whether the immunomodulatory effect of nociceptors is due to CGRP. To do this, we used daily intratumoral injection of CGRP or vehicle in sensory neuron ablated mice. CGRP-treated sensory neuron ablated mice showed a similar tumor growth and CD8 ${ }^{+}$T-cell exhaustion as nociceptor intact mice (Fig. 3H; Supp. Fig. 19; measured until day 13). We conclude that the impact of nociceptor in melanoma tumor growth control is limited to immunogenic tumors, and that such activity depends on CGRP immunomodulatory actions on CD8 ${ }^{+} \mathrm{T}$-cells.

While TRPV1 1 cre is a specific driver of sensory neurons, we aim to confirm our data using a second line of nociceptor ablated mouse. Nav1.8 is a sodium channel expressed by mechano- and thermos-sensitive neurons and $\sim 80 \%$ of all nociceptors ${ }^{22,32}$. Similar to our data with the TRPV1 1 re mouse, we found that eleven days post-B16F10 inoculation, the ablation of Nav1.8+ sensory neurons (NaV1.8cre::DTA fl/wt) slowed the tumor growth (Fig. 3I) and increased the intratumoral number of total CD8 ${ }^{+}$ T-cells (Supp. Fig. 20). The latter were also characterized by a lower relative exhaustion (Supp. Fig. 20).

Optogenetic activation of skin nociceptor neurons mediate an anticipatory immunity against microbes ${ }^{39}$, and by triggering the antidromic release of peptides, potentiates $\mathrm{skin}^{40}$ and lung ${ }^{41}$ immunity. We used transdermal illumination $(3.5 \mathrm{~ms}, 10 \mathrm{~Hz}, 478 \mathrm{~nm}$, $100 \mathrm{~mW}$, delivering $\sim 2-6 \mathrm{~mW} / \mathrm{mm}^{2}$ to a $0.39 \mathrm{NA}$ fiber placed $5-10 \mathrm{~mm}$ from the skin, for $20 \mathrm{~min}$ ) to stimulate tumor-innervating Nav1.8 neurons optogenetically NaV1.8 $\mathrm{Cre}:: \mathrm{ChR} 2^{\mathrm{fl} / \mathrm{wt}}$ mice. In comparison with light-insensitive littermate control mice, we found that eleven days post-B16F10 inoculation, blue light-stimulation of NaV1. $8^{\mathrm{Cre}}: . \mathrm{ChR}{ }^{\mathrm{f} / \mathrm{wt}}$ mice enhanced B16F10 tumor growth (Fig. 3I), decreased infiltration of total $\mathrm{CD} 8^{+}$T-cells and increased their exhaustion (Supp. Fig. 20). The optogenetic-activation of Nav1.8 neurons concomitantly increased tumor-surrounding skin CGRP levels (Supp. Fig. 20). These data link NaV1.8 ${ }^{+}$ neuron-mediated increases in CGRP levels with enhanced CD8 ${ }^{+}$T-cell exhaustion and increased B16F10 tumor growth.

The neonatal/embryonic ablation of neuronal subsets (Nav1.8 or TRPV1) may lead to compensatory changes. To circumvent this potential shortcoming, we silenced neurons using Botulinum toxin A (BoNT/A), a neurotoxic protein produced by Clostridium botulinum, which acts by cleaving SNAP2542. BoNT/A caused the long-lasting (20 days) abolition of neurotransmitter release from skin-innervating neurons ${ }^{30}$. BoNT/A reduces tumor growth in prostate cancer ${ }^{2}$ and blocks nociceptor-mediated modulation of neutrophils during skin infection ${ }^{30}$. Here, we found that BoNT/A administration $(25 \mathrm{pg} / \mathrm{\mu l}$; $50 \mu \mathrm{l} ; 5$ i.d. sites) one and three days prior to B16F10-OVA cell inoculation reduced subsequent tumor growth and intra-tumoral CD8 ${ }^{+}$T-cell exhaustion (Fig. 4A-B, Supp. Fig. 21-22) when measured 16 days post-tumor inoculation. BoNT/A treatment concomitantly increased the intra-tumoral $C D 8^{+} \mathrm{T}$-cell counts and preserved their cytotoxic potential (IFNy, Supp. Fig. 21-22). BoNT/A-mediated tumor-innervating neuron silencing also reduced YUMMER1.7 tumor outgrowth (Supp. Fig. 23; measured on day 13). 
We next tested the anti-tumor efficacy of a proven nociceptor-selective silencing strategy ${ }^{43}$. This protocol uses large pore ion channels (TRPV1) as cell-specific drug-entry ports to deliver QX-314, a charged and membrane-impermeable form of lidocaine, to block voltage-gated sodium (Nav) channels. During inflammation, similar to that observed in tumor microenvironments, these ion channels open, allowing QX-314 to permeate into these neurons resulting in a long-lasting electrical blockade ${ }^{22}$. As observed seventeen days post-B16F10 inoculation, QX-314-mediated sensory neuron silencing (0.3\%; daily i.d. surrounding the tumor) reduced melanoma growth ( $\sim 3.3-$ fold), melanoma weight ( $\sim 5.2$-fold), and the proportion of PD1 ${ }^{+} \mathrm{Lag}^{3}{ }^{+} \mathrm{Tim}^{+} \mathrm{CD} 8^{+}$ T-cells ( 15-fold; Fig. 4C-D; Supp Fig 24-25). The nociceptor silencing also increased the intra-tumor numbers of CD8 ${ }^{+}$Tcells and preserved their cytotoxic potential (IFNy, TNFa; Supp. Fig. 24-25). In separate groups of mice, we found that vehicleexposed B16F10-bearing mice succumb at a 2.7-fold higher rate $(p \leq 0.0001)$ than QX-314-exposed mice $(0.37$ hazard ratio; Fig. 4E; measured until day 19 and determined by reaching a volume of $\sim 800 \mathrm{~mm}^{3}$ or other ethical endpoints). Pharmacological silencing of nociceptors with either charged sodium channel blockers or Botox variants, could therefore, be a novel strategy for reducing melanoma growth.

Sixteen days post-B16F10 inoculation, we found increased CGRP secretion in ex vivo skin explants, which further enhanced by capsaicin stimulation (Fig. 4F). This raise in CGRP levels was absent in B16F10-incoulated skin in mice whose nociceptor neurons were silenced by QX-314 or by BoNT/A (Fig. 4F) suggesting that the anti-tumoral action of pharmacological nociceptor silencing depends on preventing CGRP-mediated CD8 ${ }^{+}$T-cells exhaustion. To specifically test this, we administered the selective RAMP1 antagonist BIBN4096, which blocks neuro-immune interplay during microbe infections and rescues host antibacterial activity ${ }^{29}$. As measured on day 16, BIBN4096 (5 mg/kg, i.p.; QAD) reduced B16F10 growth ( 1.5-fold), tumor weight ( 1.9-fold), and the proportion of PD1 $1^{+}$agg $^{+}{ }^{-}$im $^{+}{ }^{+}$CD8 ${ }^{+}$T-cells ( 2.5-fold; Fig. 4G-H; Supp. Fig. 26-27). In parallel, RAMP1 blockade increased the intra-tumor number of total ( $\sim$-fold) and IFNy ${ }^{+}\left(\sim 3-\right.$ fold) $\mathrm{CD}^{+} \mathrm{T}$-cells (Supp. Fig. 26-27). In separate groups of mice, we found that vehicle-exposed B16F10-bearing mice succumb at a 2.6-fold higher rate $(p \leq 0.04)$ than BIBN4096-exposed mice (0.37 hazard ratio; Supp. Fig. 27A; measured until day 19 and determined by reaching a volume of $\sim 800 \mathrm{~mm}^{3}$ or other ethical endpoints).

To specifically address whether RAMP1 is the main driver of CD8 ${ }^{+}$T-cells exhaustion, we transplanted (i.v.; $\left.2.5 \times 10^{6}\right)$ Rag $1^{-/-}$ (devoid of $B$ and T-cells) mice with either RAMP1-/ or RAMP1wt $C D 8^{+}$T-cells or a 1:1 mix of RAMP1-/ (CD45.2) and RAMP1wt (CD45.1) CD8 ${ }^{+} \mathrm{T}$-cells and retrieved similar number of CD8 T-cells across the three groups (Supp. Fig. 28). As measured ten days post tumor-inoculation, B16F10-OVA tumor growth (Fig. 4I) and volume ( 2.1-fold; Fig. 4J) was lower in mice that received the RAMP1-l- $\mathrm{CD} 8^{+}$T-cells as compared to RAMP1 ${ }^{\mathrm{wt}} \mathrm{CD} 8^{+} \mathrm{T}$-cell transplanted mice. In addition, the relative proportion of intra-tumor PD1 ${ }^{+} \mathrm{Lag}^{+}{ }^{+} \mathrm{Tim} 3^{+} \mathrm{CD} 8^{+} \mathrm{T}$-cells was also lower in RAMP1/- - transplanted Rag1 ${ }^{--}$mice (Fig 4K; Supp. Fig. 29). In the RAMP1 ${ }^{\text {wt }}$ and RAMP1 ${ }^{-/} \mathrm{CD} 8^{+}$T-cells co-transplanted (1:1 mix) Rag1-/- mice, we found that within the same tumor, the relative proportion of intra-tumor $\mathrm{PD} 1^{+} \mathrm{Lag}^{+} \mathrm{Tim}^{+} \mathrm{CD}^{+} \mathrm{T}$-cells was lower in RAMP1-- $\mathrm{CD} 8^{+} \mathrm{T}$-cells than $\mathrm{RAMP}$ 1 $^{\text {wt }} \mathrm{CD} 8^{+} \mathrm{T}$ cells (Fig 4L; Supp. Fig. 30). Next, we RNA-sequenced FACS-purified RAMP1 ${ }^{\text {wt }}$ and RAMP1-l- CD8 ${ }^{+}$T-cells from these mice and confirmed that intra-tumoral RAMP1-- CD8 ${ }^{+}$T-cells express less exhaustion markers (Pd1, Lag3, Tim3) compared to their RAMP1 ${ }^{\text {wt }}$ counterparts (Supp. Fig. 31). These changes were not observed amongst LN resident CD8 ${ }^{+}$T-cells (Supp. Fig. 31). RAMP1 ${ }^{--} \mathrm{CD} 8^{+}$T-cells are, therefore, protected from undergoing nociceptor-induced exhaustion, safeguarding their anti-tumor responses.

Whether RAMP1 expression control patients' $\mathrm{CD}^{+} \mathrm{T}$-cell exhaustion is unknown. To answer this, we turned to unbiased singlecell RNA-sequencing of human melanomas, in which we found $\sim 1 \%$ of tumor-Infiltrating CD8+ T-cells expressed RAMP144. When grouped together, patients' melanoma-infiltrating RAMP1 ${ }^{+} \mathrm{CD} 8^{+} \mathrm{T}$-cells overexpressed the immune checkpoint receptors PD1, Tim3, Lag3, CTLA4, CD27 which was coupled with a loss of the pro-proliferative cytokine IL-2 (Fig. 4M). This finding is replicated in another single cell RNA-sequencing cohort ${ }^{45}$ (Supp. Fig. 32). Such an expression profile resembles functional exhaustion of effector CD8 ${ }^{+}$T-cells, and confirms that RAMP1 controls patient' CD8 ${ }^{+}$T-cell exhaustion.

Melanoma biopsies also show increased expression ( $p \leq 0.05)$ of Tubb3, Tac1, Trka as well as Calca (Supp. Fig. 33), when compared with benign nevi ${ }^{46-49}$. Finally, increased tumor expression of RAMP1 (Fig. 4N; 459 patients (40)) correlated ( $p \leq 0.05$ ) with reduce patients' survival. Overexpression of the melanoma-secreted nociceptor-activating SLPI, as well as other nociceptor neuron markers (Nav1.7), also correlate with a worse patient prognosis (Supp. Fig. 34). Similar to our pre-clinical mouse modelling, these human data imply that nociceptor-produced CGRP drives patients intra-tumoral CD8 ${ }^{+} \mathrm{T}_{\text {-cells }}$ exhaustion, as we find to be the case in the mouse, fuelling cancer growth and worsening the clinical prognosis.

Blocking the activity of immune checkpoint proteins releases the cancer cell-induced "brake" on the immune system, increasing its ability to eliminate tumors ${ }^{6,8-10}$. Immune checkpoint inhibitors (ICl), including those targeting PDL1, improve clinical outcomes in patients with metastatic melanoma ${ }^{9,50-52}$, but ICl efficacy drastically varies between patients and half will not benefit ${ }^{50}$. While the reason for such variability remains unclear, we found that tumor-infiltrating $\mathrm{CD}^{+}$cells harvested from ICI resistant patients drastically overexpress RAMP1 ( 2.0-fold), ranking third highest amongst the 18-exhaustion marker probed and ahead of PD1 (1.7-fold), Lag3 (1.6-fold), Ctla4 (1.6-fold), and Tim3 (1.7-fold; Fig. 40; Supp. Fig. 35). Given that the CGRP-RAMP1 axis controls $\mathrm{CD}^{+} \mathrm{T}$-cell exhaustion and that RAMP1 negatively correlates with $\mathrm{ICl}$ responsiveness, we set out to assess the impacts of nociceptors on aPDL1 responsiveness. First, we compared aPDL1 efficacy (i.p; day $7,10,13)$ in mice whose tumors 
were inoculated on the same day. aPDL1-mediated tumor reductions were increased $\sim 2.5$-fold in nociceptor ablated mice and $\sim 5$-fold in QX-314 nociceptor-silenced mice (Supp. Fig. 36) indicating a major impact of nociceptors on immune checkpoint inhibitor activity on $\mathrm{T}$ cell function. Both these reductions were associated with an increased infiltration of tumor-specific CD8 ${ }^{+}$ T-cells which displayed reduced exhaustion (PD1 ${ }^{+} \mathrm{Lag}^{+}{ }^{+} \mathrm{Tim} 3^{+}$; Supp. Fig. 36). Second, we compared the efficacy of $\alpha$ PDL1 in mice with similar sized tumor $\left(\sim 100 \mathrm{~mm}^{3}\right)$. To achieve this, nociceptor ablated (TRPV1 $\left.{ }^{\text {cre.::DTA }}{ }^{\mathrm{f} / \mathrm{wt}}\right)$ or silenced $(\mathrm{QX}-314) \mathrm{mice}$ were inoculated $\sim 3$ days before littermate control or vehicle-exposed mice. aPDL1 (i.p; day $7,10,13$ ) mediated reduction in tumor growth increased $\sim 1.5$-fold in nociceptor ablated mice, and $\sim 5$-fold when administered in combination with QX-314 (Supp. Fig. 37). aPDL1 increased nociceptor activity ${ }^{14}$ by stimulating PD1+ neurons (Supp. Fig. 38) which promote neuropeptide release and subsequent TIL exhaustion. Silencing nociceptors (e.g. with QX-314) may therefore augment $\alpha \mathrm{PDL} 1$ efficacy by limiting its pro-nociceptive effects and, in turn, safeguarding the host anti-tumor immunity. Blocking nociceptor action via CGRP on RAMP1+ $1^{+} \mathrm{CD} 8^{+} \mathrm{T}$-cells represents therefore, a potential complementary treatment for enhancing immune checkpoint blockers activity and perhaps reducing non responsivity to $\mathrm{ICI}$ agents.

Our rodent data shows that B16F10 melanoma tumors are innervated by Nav1.8 and TRPV1 expressing sensory neurons and that B16F10-released SLPI activates these tumor-innervating nociceptors. Genetic ablation of Nav1.8 or TRPV1 decreases B16F10 tumor growth and rescues $\mathrm{CD}^{+}$T-cells exhaustion while the optogenetic activation of Nav1.8 nociceptors or the exogenous administration of CGRP has the opposite effects. These actions are restricted to immunogenic tumors (B16F10OVA, YUMMER1.7) and are absent in non-immunogenic tumors (YUMM1.7) or in the absence of CD3s. Blocking tumorinnervating nociceptor release of CGRP, using QX-314 or BoNT/A, or blocking its receptor RAMP1, using BIBN4096, decreases tumor growth and prevents CD8 ${ }^{+}$T-cell exhaustion.

The targeted mouse genetic (Nav1.8 ${ }^{\mathrm{Cre}}:: \mathrm{ChR} 2^{\mathrm{f} / \mathrm{wt}}$; Nav1.8 $\mathrm{Cre}:: \mathrm{DT} \mathrm{A}^{\mathrm{f} / \mathrm{wt} ;}$ TRPV1 ${ }^{\mathrm{Cre}}:: \mathrm{DT} \mathrm{A}^{\mathrm{f} / \mathrm{wt} ;}$ RAMP1 ${ }^{-/-}$CD8 T cells) and pharmacological (CGRP, BIBN4096, CGRP8-37, capsaicin, QX-314, BoNT/A) approaches used here are specific for nociceptors 22,29,31,32,39-41,53-61. Thus, BoNT/A, QX-314, and BIBN4096 did not impact either B16F10 survival or CD8 ${ }^{+}$T-cell function in vitro (Supp. Fig. 39-41) - only working in the setting of tumor innervation in vivo. Furthermore, genetically ablated or pharmacologically silenced nociceptors also abolished B16F10-induced mechanical and thermal pain (hindpaw-inoculated) and itching ( $\sim 30 \%$ of flank-inoculated B16F10 cells; Supp. Fig. 42). These effects were associated with a decrease in intratumoral CGRP (Fig. 4F). BoNT/A-silencing or CGRP antagonism (BIBN4096) only act in the presence of intact nociceptors, as they do not impact tumor growth when administered to sensory neuron ablated (TRPV1 ${ }^{\mathrm{Cr}}::$ DTA ${ }^{\mathrm{fl} / \mathrm{wt}}$ ) mice (Supp. Fig. 23, 43). Single-cell ${ }^{4}$ and bulk RNA-sequencing as well as microarray datasets from the Immgen database show that mouse immunocytes do not express Calca (gene encoding for CGRP), Snap25 (the molecular target of BoNT/A) or Trpv1 and Nav1.8 channels (both being required for QX-314 activity) (Supp. Fig. 5A) while B16F10 cells also display no Trpv1, Nav1.8, Snap25, Calca, or Ramp1 expression (Supp. Fig. 44).

Melanomas in patients are densely innervated by TRPV1+ nociceptor neurons and increases RAMP1 expression correlates with a worse prognosis, while its expression in $\mathrm{CD} 8^{+} \mathrm{T}$-cells is linked both to their exhaustion and resistance to ICI treatment. Two independent single-cell RNA-sequencing datasets performed on melanoma biopsies $(45,46)$ (Supp. Fig. 45-46), as well as another RNA-sequencing dataset confirm that human immunocytes do not express Trpv1, Nav1.7, Calca, or Snap25 (Supp. Fig. 47). Single-cell RNA-sequencing datasets also confirm that neither malignant cells (10 different patients; Supp. Fig. 48) nor the tumor stromal cells (cancer-associated fibroblasts, endothelial cells, etc.) express Trpv1, Nav1.7, Calca, Ramp1, or Snap25 (Supp. Fig. 45-46). The elevation in TRPV1 and Nav1.7 expression detected in patient' biopsies therefore reflect enhanced nociceptor neuron innervation and increased RAMP1 expression origins from tumor-infiltrating immunocytes.

Our data support a regulatory role for nociceptors in dampening the immune response to melanoma tumor growth through upregulation of multiple immune checkpoint receptors on cytotoxic CD8 ${ }^{+}$T-cells. Silencing tumor-innervating sensory neurons represents therefore a strategy for attenuating the immunomodulatory action of the nervous system, and promoting robust antitumor activity of the immune system (Supp. Fig. 49) and also constitutes a potential adjuvant to ICls.

Acknowledgments. The Canada Research Chair program (ST, 950-231859), Canadian Institutes of Health Research (ST and MR, 407016), the Canadian Foundation for Innovation (ST, 37439), Natural Sciences and Engineering Research Council of Canada (ST; RGPIN-2019-06824) and the Brain Canada Foundation (4758), Health Canada and the Azrieli Foundation (ST) and National Institute of Health (NIH) grants R35-NS10507603 (CJW) financed the work. MB, MA, and TE hold a scholarship from the Fonds de Recherche Sante Quebec (FRQS) and CAPES. We want to thank Dr. Ana C. Anderson for initial discussions, Dr. Aviv Regev, and Dr. Livnat Jerby-Arnon for helping with the analysis of the single-cell RNA sequencing datasets.

Author contributions. MB, MA, TE, AM, HM, AP, BD, CTL, DWV, SH, LJ, HD, KR, SNC, CRS, SLF, AA and ST conducted the experiments. MB, SH, CJS, MR, MR, JT, SDR, NG, RD, CJW, PDV and ST designed the study. MB, MA, SDR, PDV, CJW and ST wrote the manuscript.

Declaration of interests. ST and CJW have an equity stake in Nocion Therapeutics. 

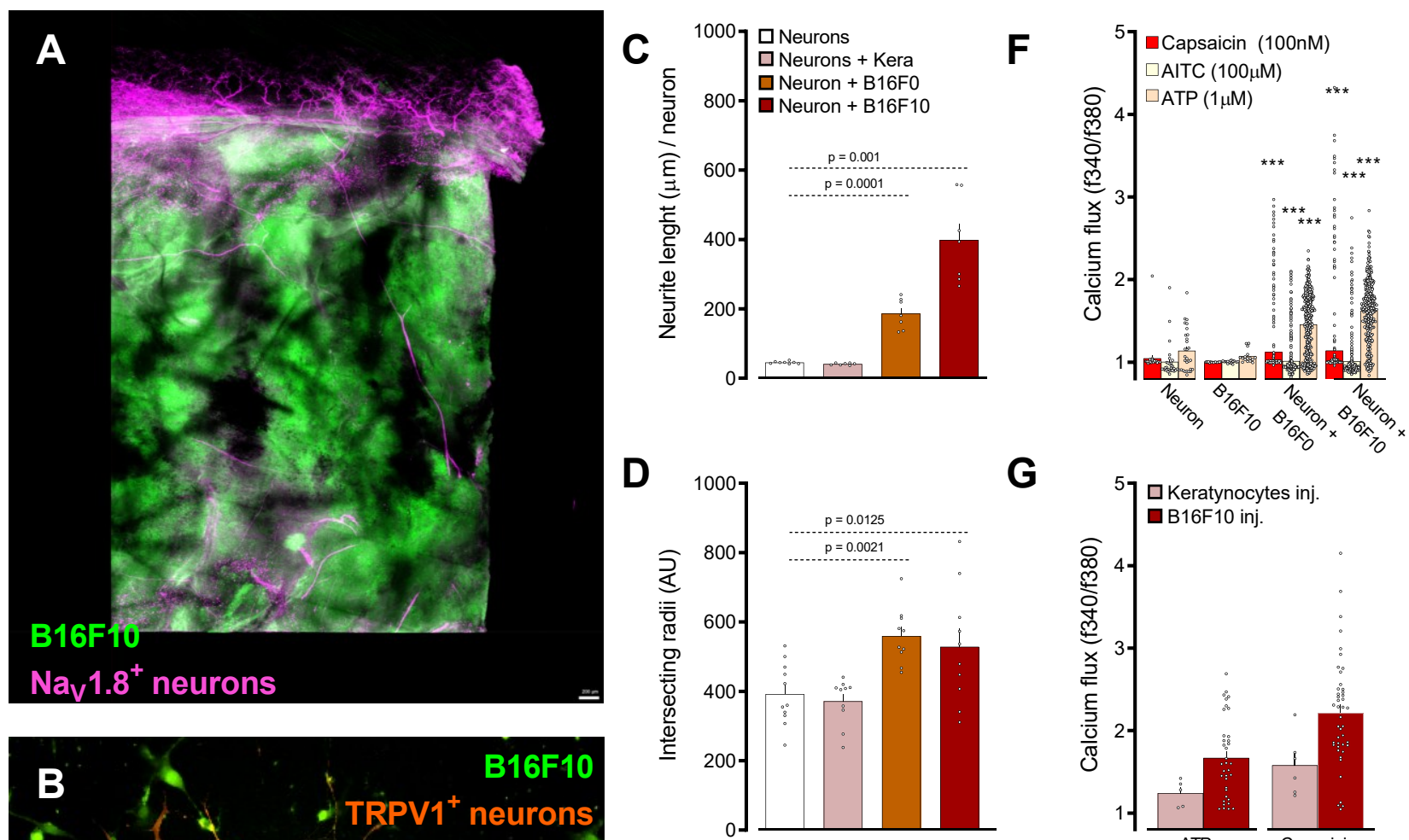

$\mathbf{G}$
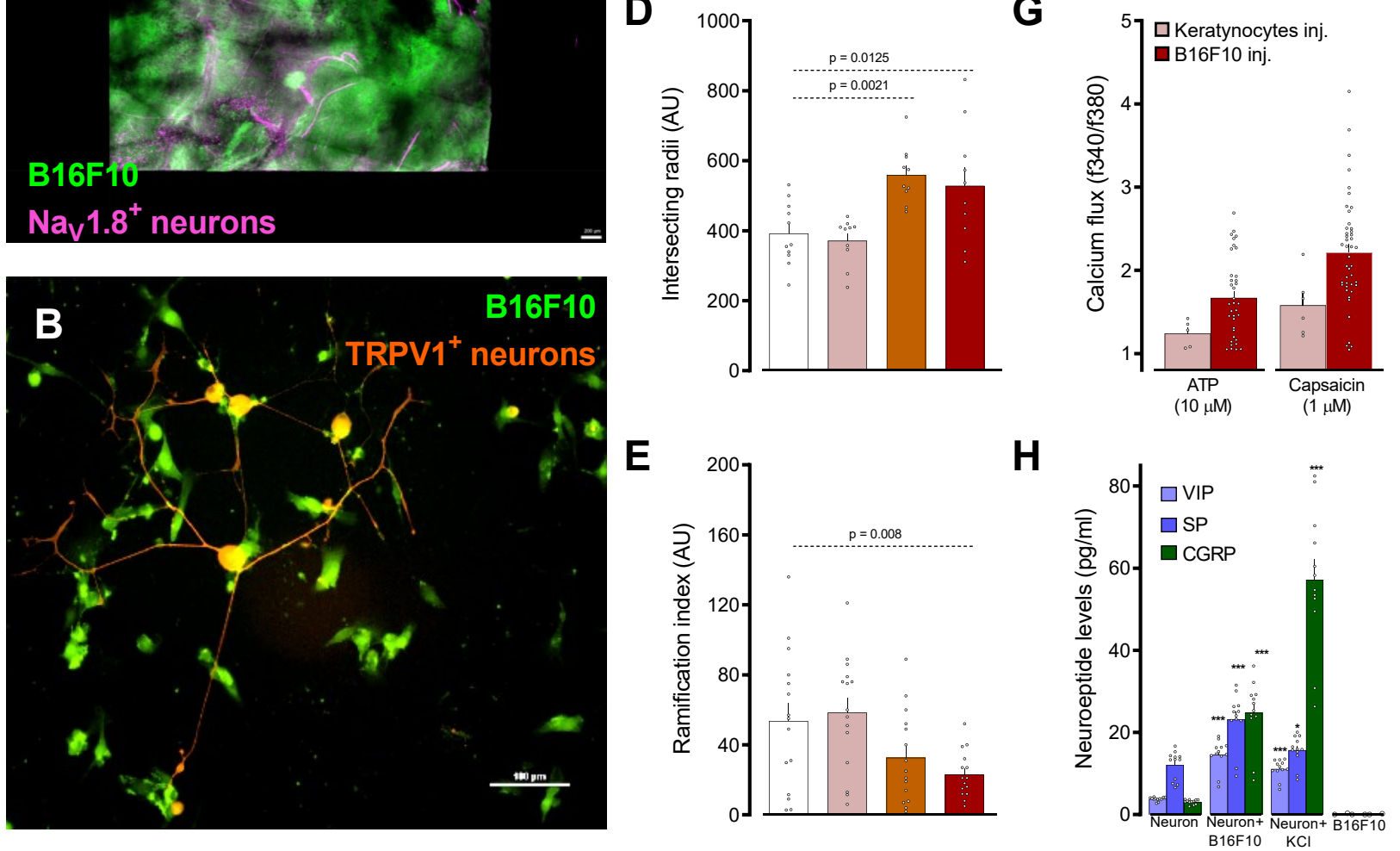

Figure 1: Melanoma sensitizes nociceptors. Nociceptor (Nav1.8cre::Td-tomato ${ }^{\mathrm{f} / \mathrm{wt}}$; magenta) reporter mice hindpaw were inoculated with B16F10-eGFP cancer cells ( $10^{5}$ cells; i.d.; green). iDISCO image of Nav1.8 nerve fibers (magenta) innervating B16F10eGFP-inoculated mouse skin after twenty-two-days (A). TRPV1+ nociceptors (red; TRPV1Cre::td-tomatof ${ }^{\text {fl/wt }}$ ) co-cultured with B16F10-eGFP have longer neurites (B-D), reduced arborization (E), form neuro-neoplastic contacts (B), then when cultured alone or with non-tumorigenic keratinocytes (B-E). In co-culture, B16F0 or B16F10 cells sensitize the response of nociceptors to capsaicin $(100 \mathrm{nM})$, AITC $(100 \mu \mathrm{M})$, ATP $(1 \mu \mathrm{M})$ measured by calcium influx $(\mathrm{F})$. Low concentration of the ligands induced minimal response in control neurons, while B16F10 induced enhanced responses to ATP (F). L3-L5 DRG neurons harvested from mice 2-weeks post-implantation with B16F10- or non-tumorigenic keratinocytes in the hindpaw were cultured and calcium flux to ligands tested. In comparison to keratinocytes, neurons from tumor-bearing mice were more sensitive to ATP $(10 \mu \mathrm{M})$, and capsaicin $(1 \mu \mathrm{M} ; \mathbf{G})$. Neurons co-cultured with B16F10 cells release the neuropeptide CGRP (H). B16F10 cells alone do not release neuropeptide $(\mathbf{H})$. Data are shown as mean \pm S.E.M; One-way ANOVA post hoc Bonferroni $(\boldsymbol{C}-\boldsymbol{F}, \boldsymbol{H})$ or unpaired Student's t-test (G); $p$ values are shown in the figure. Scale bar $=200 \mu m(\boldsymbol{A})$ or $100 \mu m(B)$. Nav1. $8^{+}$nociceptors are labeled in magenta $(\boldsymbol{A})$, TRPV1 $^{+}$nociceptors are labeled in red (B) while B16F10-eGFP cells are labeled in green (A-B). 

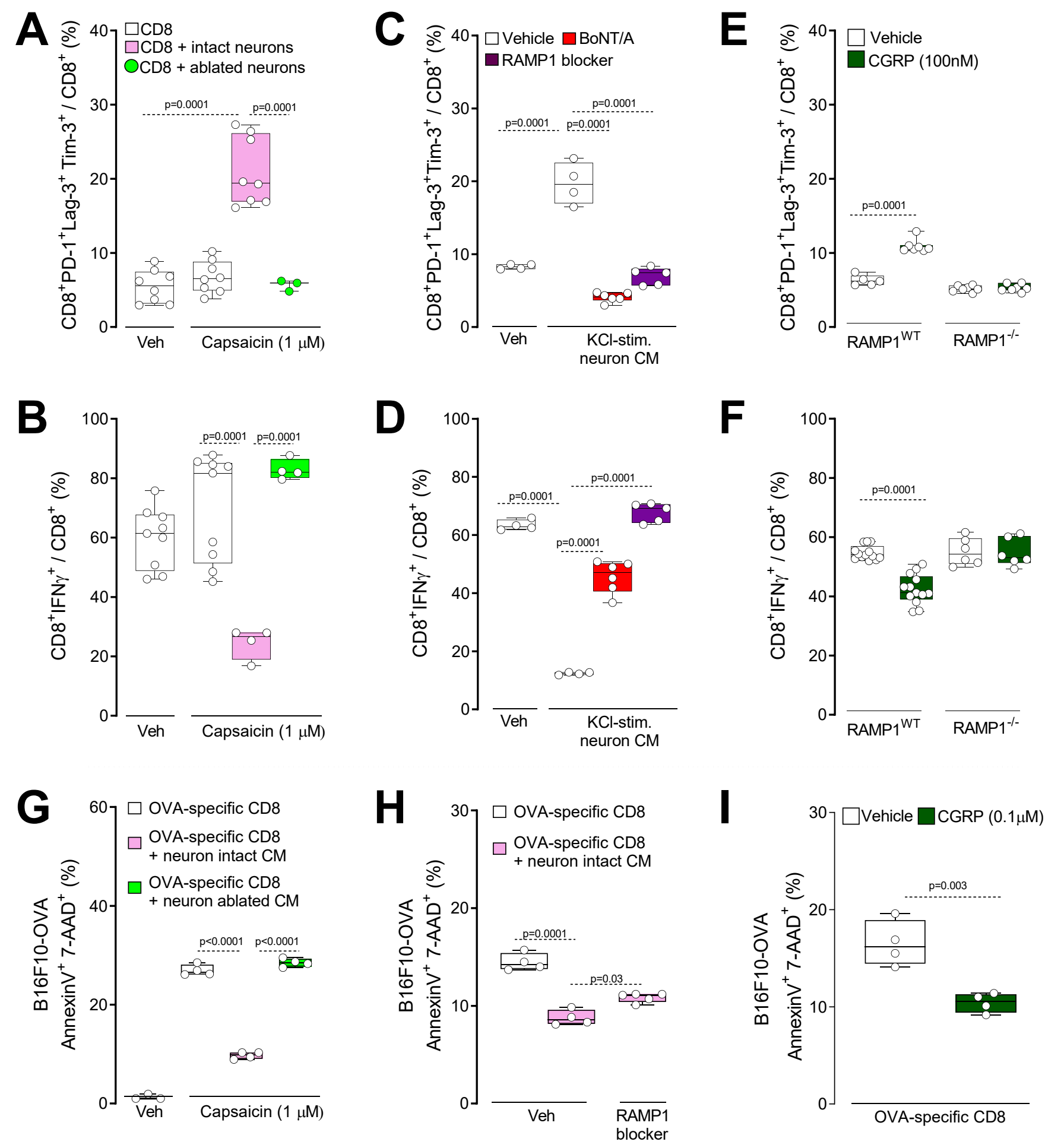

Figure 2: Nociceptor-released neuropeptides drive cytotoxic $\mathrm{CD}^{+} \mathrm{T}$-cell exhaustion. Splenocytes-isolated $\mathrm{CD} 8^{+} \mathrm{T}$-cells were cultured under Tc1-stimulating conditions for $48 \mathrm{~h}$ to generate cytotoxic CD8 ${ }^{+} \mathrm{T}$-cells $(\mathrm{A}-\mathrm{I})$. In the presence of IL-2

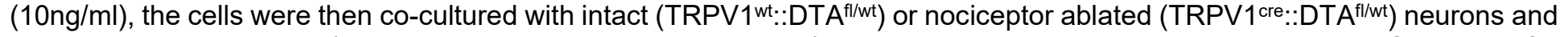
stimulated with capsaicin $(1 \mu \mathrm{M}$, challenged once every two days) or its vehicle. Upon co-culture with naive DRG neurons for an additional $96 \mathrm{~h}$, capsaicin-stimulation increased the proportion of PD $1^{+} \mathrm{Lag} 3^{+} \mathrm{Tim} 3^{+}$cells $(\mathbf{A})$, and reduced the proportion of IFNY ${ }^{+}$cells (B). This effect was absent when exposed to nociceptor ablated DRG neurons (A-B). As observed after 96h stimulation, conditioned media (1:2 dilution, challenged once every two days) harvested from $\mathrm{KCl}(50 \mathrm{mM})$-stimulated DRG neurons increased proportion of Tc1-stimulated cytotoxic CD8 ${ }^{+}$T-cells PD1 ${ }^{+} \mathrm{Lag}^{+}{ }^{+} \mathrm{Tim}^{+}$cells $(\mathbf{C})$ and reduced IFNY ${ }^{+}$cells (D). 
These effects were prevented when cytotoxic CD8 $8^{+}$-cells were co-exposed to a RAMP1 blocker (CGRP8-37; $\left.2 \mu \mathrm{g} / \mathrm{ml}\right)$ or when challenged (1:2 dilution; challenged once every two days) with fresh $\mathrm{KCl}$-induced conditioned media from BoNT/A-silenced neurons (100pg/200 $\mathrm{\mu l}$; C-D). When directly stimulated with CGRP $(0.1 \mu \mathrm{M}$; challenged once every two days) for $96 \mathrm{~h}$, wildtype cytotoxic $\mathrm{CD} 8^{+}$T-cells showed an increased proportion of $\mathrm{PD} 1^{+} \mathrm{Lag}^{+} \mathrm{Tim} 3^{+}$cells $(\mathbf{E})$, but reduced the proportion of IFNY ${ }^{+}(\mathbf{F})$ cells. These effects were absent when treating cytotoxic $\mathrm{CD} 8^{+}$T-cells harvested from RAMP1/- mice (E-F). Splenocytesisolated $\mathrm{CD} 8^{+} \mathrm{T}$-cells from naive OT-1 mice were cultured under $\mathrm{T}_{\mathrm{c} 1}$-stimulating conditions for $48 \mathrm{~h}$. B16F10-mCherry-OVA cells $\left(1 \times 10^{5}\right.$ cells) were then cultured with or without OT- 1 cytotoxic CD8 ${ }^{+}$T-cells $\left(4 \times 10^{5}\right.$ cells; G-I). Tc1-stimulated OT1-CD8 ${ }^{+}$T-cells $^{-}$ lead to B16F10-OVA cell apoptosis (AnnexinV+7AAD+; G, measured after 48h; H-I, measured after 24h). B16F10-mCherryOVA cells elimination by cytotoxic $C D 8^{+} \mathrm{T}$-cells was reduced when the co-cultures were challenged (1:2 dilution; once daily for two consecutive days) with fresh conditioned media harvested from capsaicin (1 $\mu \mathrm{M})$-stimulated naive DRG neurons

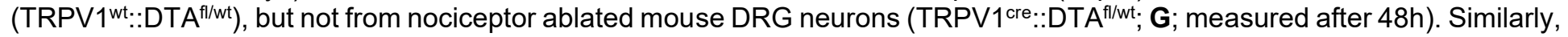
$\mathrm{KCl}(50 \mathrm{mM})$-stimulated naive DRG neurons conditioned media (1:2 dilution) reduced B16F10-mCherry-OVA apoptosis $(\mathbf{H}$; measured after 24h). This effect was blunted when the cells were co-exposed to the RAMP1 blocker CGRP $8-37\left(\mathbf{H}_{2} ; \mu \mathrm{g} / \mathrm{ml}\right.$; measured after 24h). CGRP $(0.1 \mu \mathrm{M})$ challenges also reduced B16F10-OVA cell apoptosis (I; measured after 24h). Data are shown as mean \pm S.E.M; One-way ANOVA posthoc Bonferroni $(\boldsymbol{A}-\boldsymbol{H})$; unpaired Student's $t$-test (I); $p$ values are shown in the figure. 

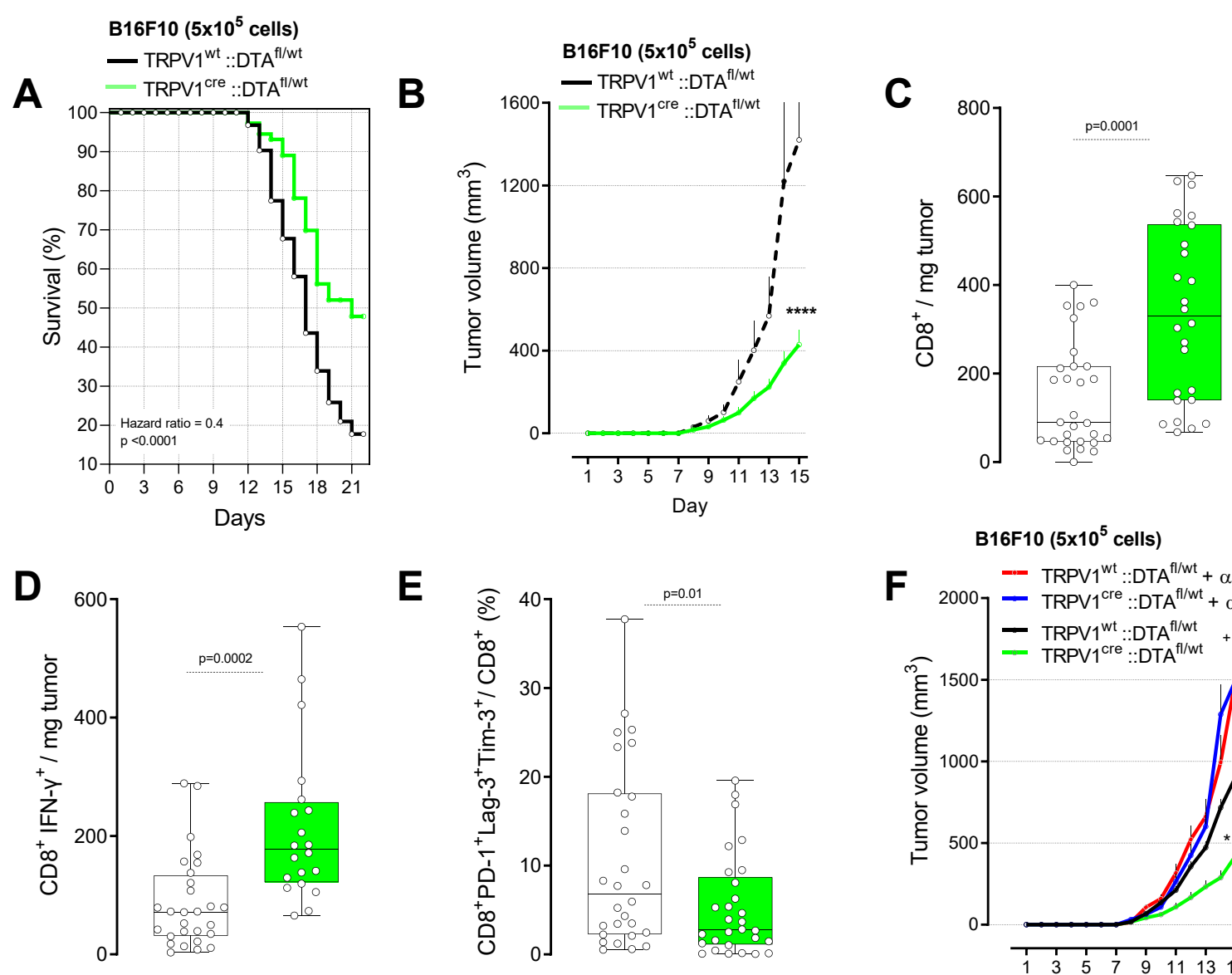

E
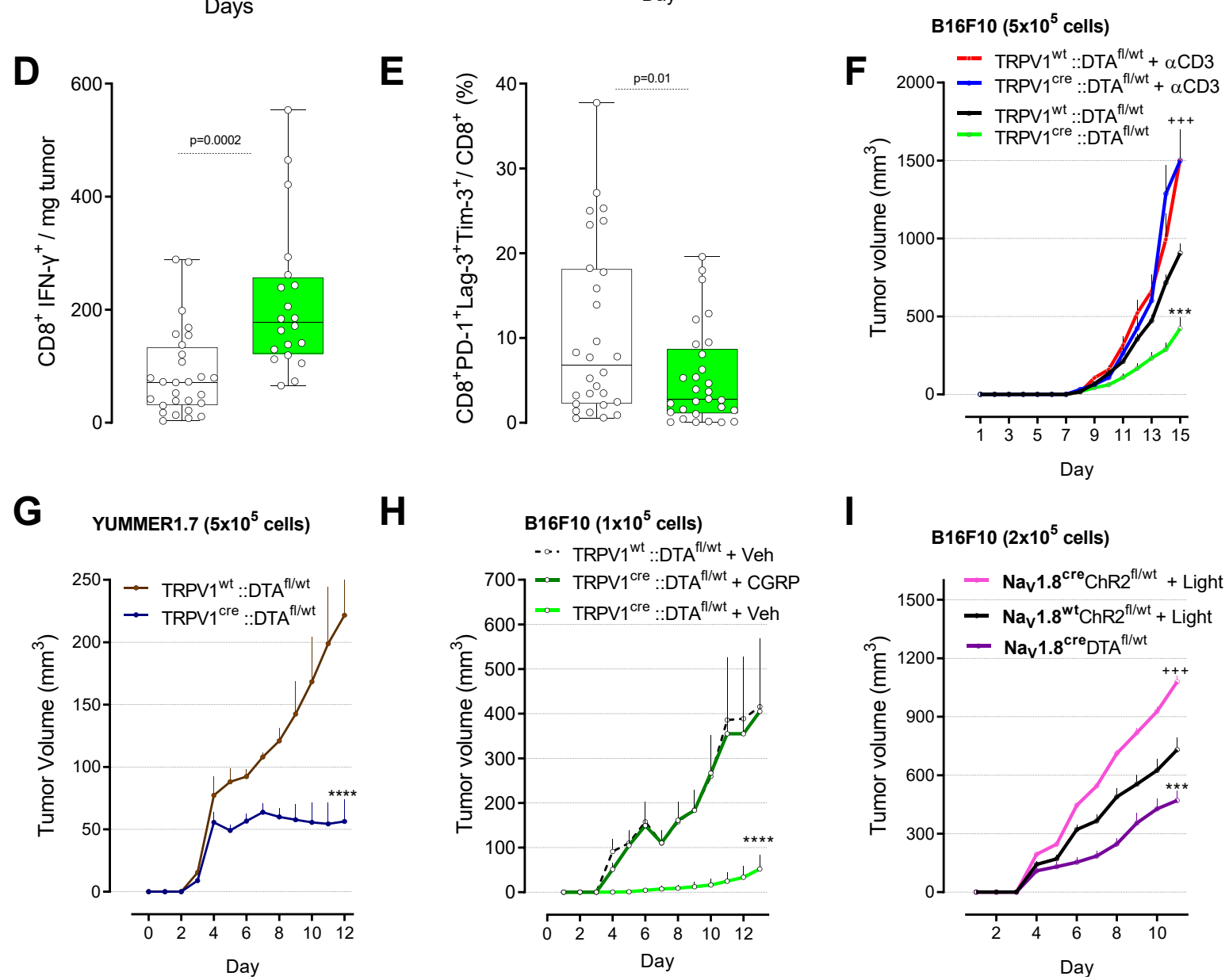

H
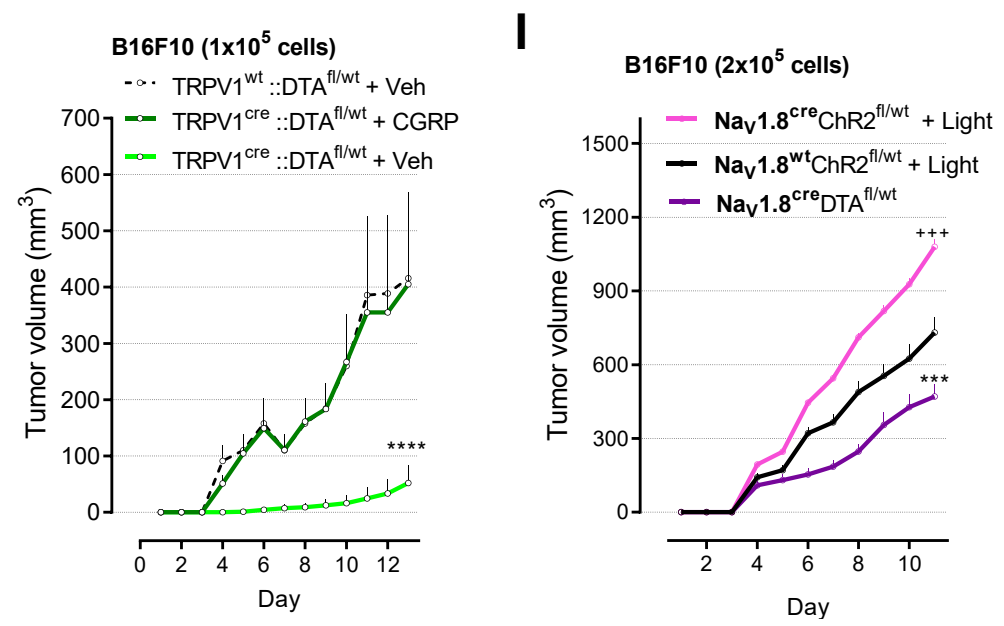

Figure 3: Genetic elimination of nociceptors reduces TIL exhaustion. Orthotopic B16F10-mCherry-OVA (5x105 cells; i.d.) median length of survival was increased by $\sim 250 \%$ (A; Mantel-Haenszel Hazard Ratio; measured on day 22) in nociceptor ablated mice (TRPV1 1 Cre::DTA ${ }^{\mathrm{f} / \mathrm{wt}}$ ). In separate group of mice and fifteen days post tumor inoculation, sensory neuron ablated mice have reduced tumor growth (B), increased tumor infiltration of total (C) and IFNy ${ }^{+} \mathrm{CD}^{+} \mathrm{T}$-cells (D); while the proportion of $\mathrm{PD}^{+}{ }^{+} \mathrm{Lag}^{+}{ }^{+} \mathrm{Tim}^{+} \mathrm{CD}^{+} \mathrm{T}$-cells decreased $(\mathrm{E})$. The reduction in B16F10-mCherry-OVA $\left(1 \times 10^{5}\right.$ cells; i.d.) tumor growth observed in nociceptors ablated mice was absent following systemic CD3 depletion ( $F$, assessed until day 15; $\alpha$ CD3, 200 $\mu \mathrm{g} / \mathrm{mice}$; i.p.; every 3 days). Sensory neuron ablation (TRPV1Cre::DTA fl/wt) decreased the growth of YUMMER1.7 cells $\left(5 \times 10^{5}\right.$ cells; i.d.); an immunogenic version of a Braf ${ }^{6000}{ }^{2} \mathrm{Ckn}^{2} \mathrm{a}^{-/}{ }^{-} \mathrm{Pten}^{-/}$melanoma cell line (G; assessed until day 13). Orthotopic B16F10-mCherry-OVA cells $\left(1 \times 10^{5}\right.$ cells; i.d.) were inoculated into 8-week-old male and female intact or nociceptor ablated mice. Administering recombinant CGRP (100nM, i.d., once daily) rescues tumor growth in nociceptor ablated mice $(\mathbf{H}$; assessed until day 12). Daily optogenetic activation $\left(3.5 \mathrm{~ms}, 10 \mathrm{~Hz}, 478 \mathrm{~nm}, 100 \mathrm{~mW}\right.$, giving approx. $2-6 \mathrm{~mW} / \mathrm{mm}^{2}$ with a $0.39 \mathrm{NA}$ fiber placed 5-10 $\mathrm{mm}$ from the skin, for $20 \mathrm{~min})$ of B16F10-inoculated skin $\left(2 \times 10^{5}\right.$ cells; i.d.) in light-sensitive NaV1.8 ${ }^{\mathrm{Cre}}:: \mathrm{ChR} 2^{\mathrm{fl} / \mathrm{wt}}$

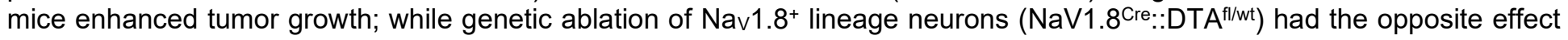
(I; assessed until day 11). Data are shown as mean \pm S.E.M; Mantel-Cox regression (A), Two-way ANOVA posthoc Bonferroni (B, F-I); or unpaired Student's t-test (C-E); $p$ values are shown in the figure. 
A

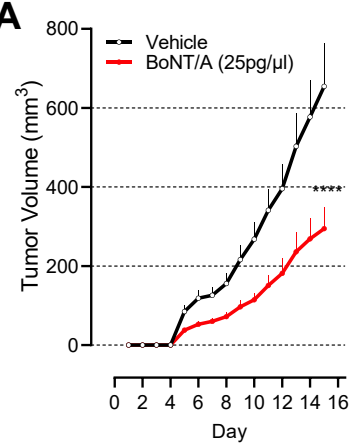

E

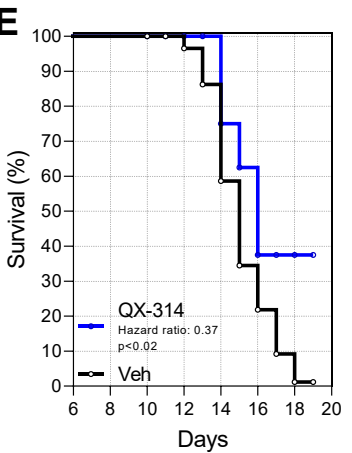

I

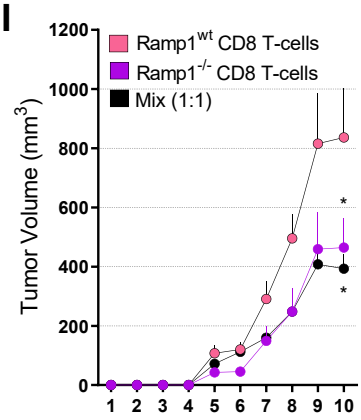

M

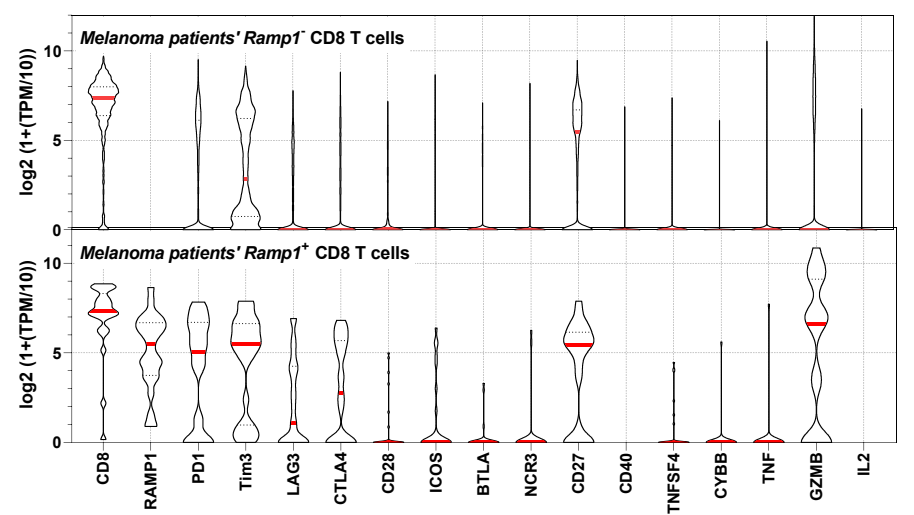

C

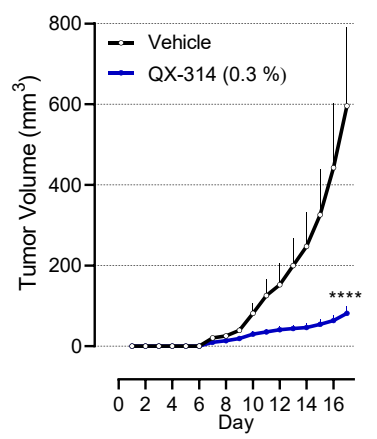

F

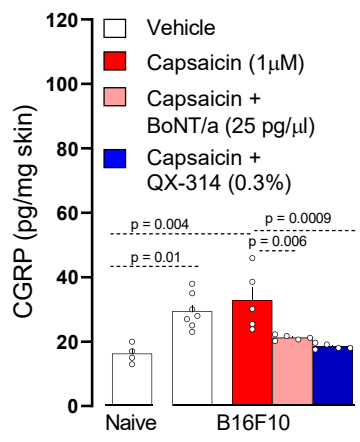

J

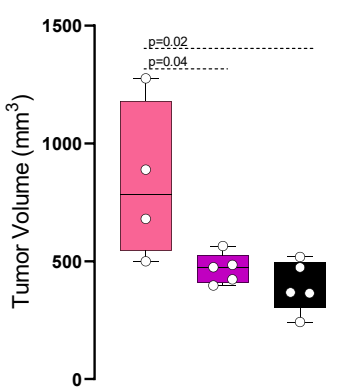

G

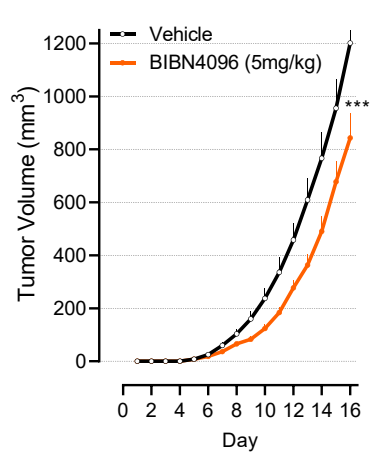

K

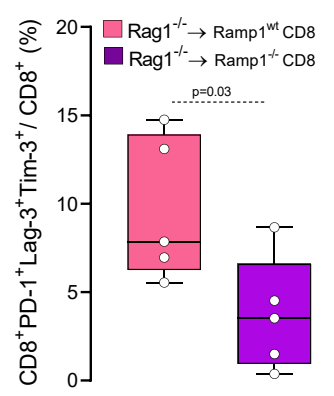

$\mathbf{N}$

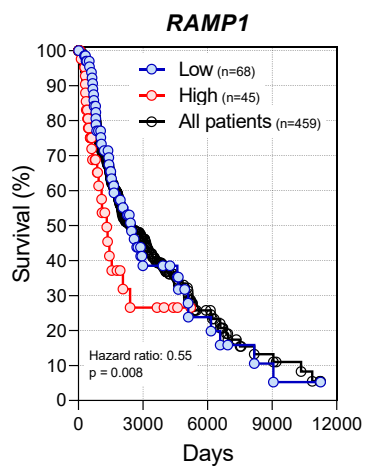

D

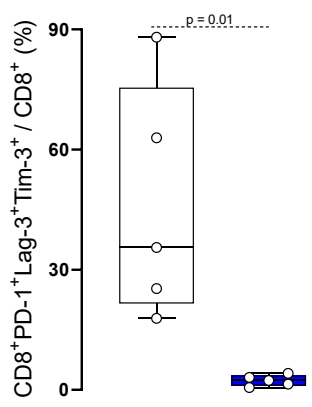

H

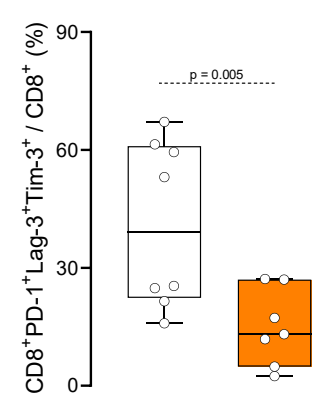

L

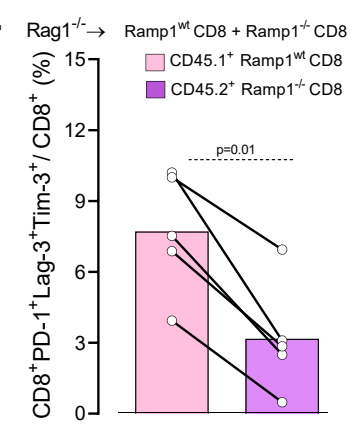

0

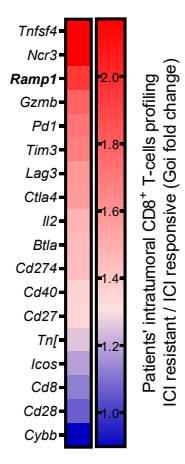

Figure 4: Silencing tumor-innervating nociceptors rescues anti-tumor immunity. Sixteen (A-B) or seventeen (C-D) days post-B16F10-mCherry-OVA $\left(5 \times 10^{5}\right.$ cells; i.d.) inoculation, tumor growth $(\mathbf{A}, \mathbf{C})$ and the proportion of PD1+Lag3 ${ }^{+} \mathrm{Tim}^{+} \mathrm{CD}^{+} \mathrm{T}^{-}$ cells (B, D) were reduced in mice whose tumor-innervating neuron were silenced by BoNT/A (A-B; $25 \mathrm{pg} / \mu \mathrm{l} ;$ twice, i.d.) or QX314 (C-D, $0.3 \%$, i.d., q.d.). In separate group of mice, we found that tumor-innervating nociceptor silencing (QX-314) prolonged the median length of survival by $\sim 2.7$-fold ( $\mathrm{E}$; assessed until day 19 and determined as volume $\geq 800 \mathrm{~mm}^{3}$ ). CGRP levels were increased in B16F10 tumor surrounding skin explant (measured on day 15) in comparison to control skin; an effect further

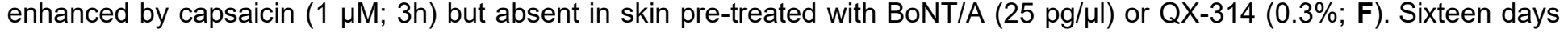

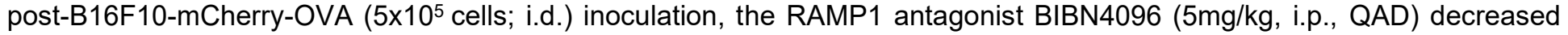
tumor growth $(\mathbf{G})$ and reduced proportion of intra-tumor $\mathrm{PD}^{+}{ }^{+} \mathrm{Lag}^{+}{ }^{+} \mathrm{Tim}^{+}{ }^{+} \mathrm{CD} 8^{+} \mathrm{T}$-cells $(\mathbf{H})$. Naive splenocytes $\mathrm{CD} 8^{+} \mathrm{T}^{-c e l l s}$ were FACS-purified from RAMP1wt $\left(\mathrm{CD} 45.1^{+}\right)$or RAMP1-/- $\left(\mathrm{CD} 45.2^{+}\right)$mice, cultured and amplified. 8-weeks-old female Rag ${ }^{-/-}$ 
mice were transplanted (i.v., $2.5 \times 10^{6}$ cells) with either of RAMP1-/- or RAMP1wt CD $8^{+}$T-cells or $1: 1$ mix of $5 \times 10^{6}$ RAMP1-/- and RAMP1 ${ }^{\text {wt }} \mathrm{CD} 8^{+}$T-cells. One-week post-transplantation, the mice were inoculated with B16F10-mCherry-OVA cells $\left(5 \times 10^{5}\right.$ cells; i.d.). Ten days post-B16F10 inoculation, we observed a higher tumor growth (I) and volume (J) in RAMP1WT transplanted mice than the one in RAMP1/- or RAMP1 wt $^{-}$RAMP1-/- co-transplanted mice. The relative proportion of intra-tumor PD $1^{+} \mathrm{Lag}^{+}{ }^{+} \mathrm{Tim}^{+}$ $\mathrm{CD}^{+} \mathrm{T}_{\text {-cells }}$ was lower in RAMP1-- transplanted mice $(\mathrm{K})$. Within the same tumor, the relative proportion of intra-tumor $\mathrm{PD}^{+}{ }^{+} \mathrm{Lag}_{3}{ }^{+} \mathrm{Tim}^{+}{ }^{+} \mathrm{CD} 8^{+} \mathrm{T}$-cells was also lower in RAMP1-l- CD8 ${ }^{+} \mathrm{T}$-cells than in the one found in RAMP1 ${ }^{\text {wt }} \mathrm{CD} 8^{+} \mathrm{T}$-cells $(\mathrm{L})$. Insilico analysis of single-cell RNA-sequencing of human melanoma ${ }^{44}$ revealed that intratumoral RAMP1-expressing CD8 ${ }^{+} \mathrm{T}$ cells strongly overexpressed several immune checkpoint receptors (PD1, Tim3, Lag3, CTLA-4, CD28, ICOS, BTLA, CD27) in comparison to RAMP1-negative $\mathrm{CD} 8^{+}$T-cells (M). In-silico analysis of TCGA data ${ }^{62}$ was used to correlate the survival rate of 459 melanoma patients with relative expression levels (determined by bulk RNA-sequencing) of RAMP1 found in their tumors (N). Kaplan-Meier curves show that increased RAMP1 expression (label as high; $n=45$ patients) in biopsy correlates with decreased patient survival $(p \leq 0.05)$ in comparison to all patients or in patients found with low RAMP1 expression (label as low; $\mathrm{n}=68$ patients; $\mathbf{N}$ ). Based on the clinical report, melanoma patients were identified as immune checkpoint receptor responsive or resistant ${ }^{44}(\mathbf{O})$. In-silico analysis of single-cell RNA sequencing of patients' biopsies revealed that tumor-infiltrating CD8 ${ }^{+} \mathrm{T}$ cells from immune checkpoint receptor resistant patients overexpress RAMP1 (2.0-fold), PD1 (1.7-fold), Lag3 (1.6-fold), Ctla4 (1.6-fold), and Tim3 (1.7-fold; 0). Heatmaps show average gene expression ratio found in ICl resistant CD $8^{+} T$-cells versus the one found in ICl responsive (O). Data are shown as mean \pm S.E.M; Two-way ANOVA posthoc Bonferroni (A, C, G, I), one-way ANOVA posthoc Bonferroni (F), Mantel-Cox regression $(\boldsymbol{E}, \boldsymbol{N})$, or unpaired Student's t-test $(\boldsymbol{B}, \boldsymbol{D}, \boldsymbol{H}, \boldsymbol{J}, \boldsymbol{K}, \boldsymbol{L}) ; p$ values are shown in the figure. 

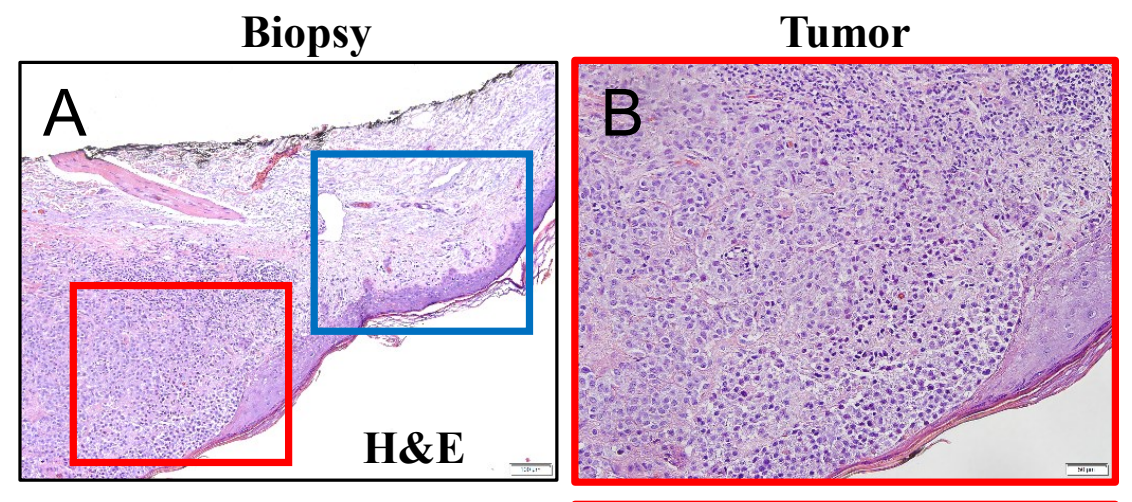

Intact tissue (margin)
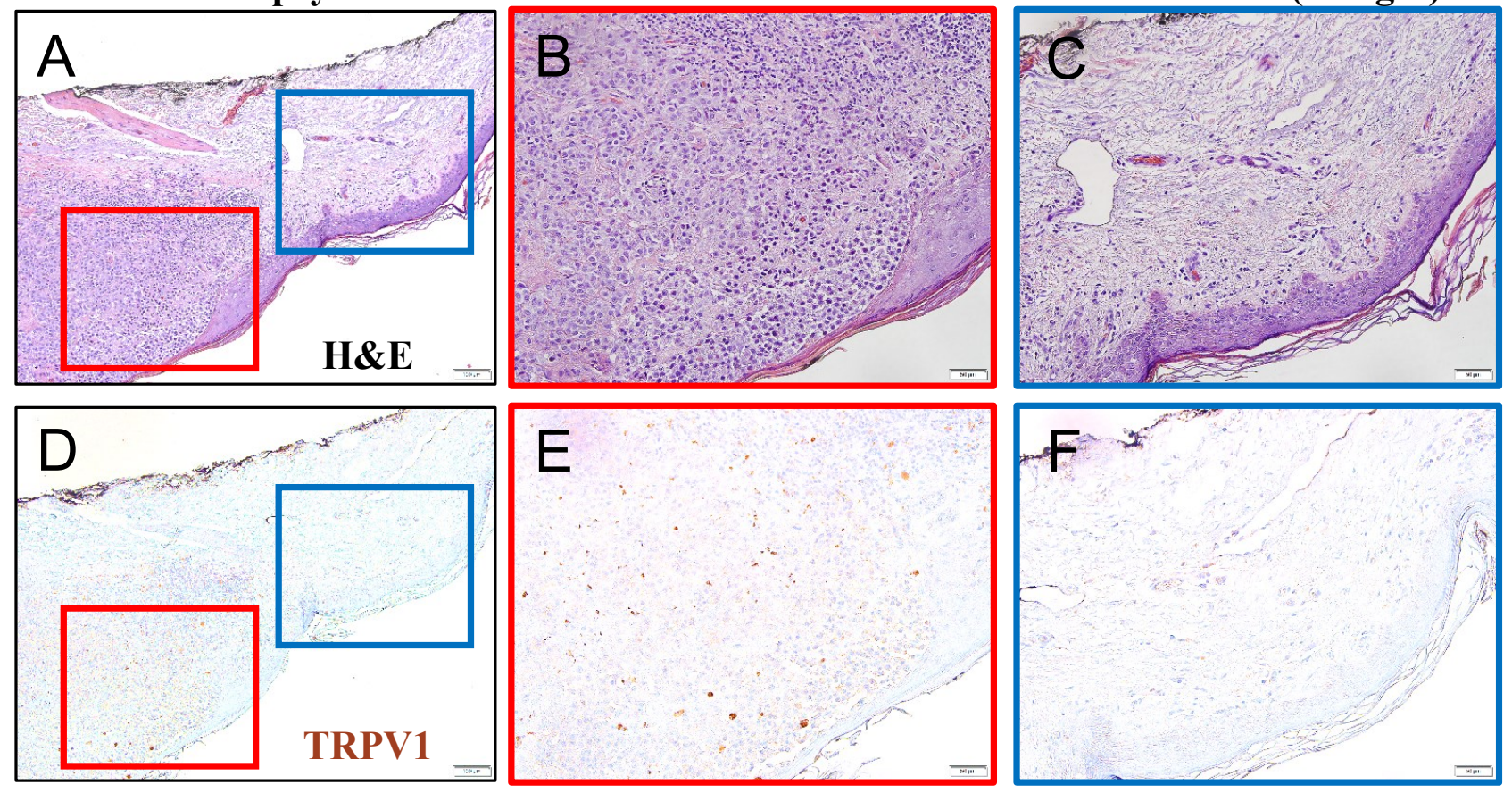

G

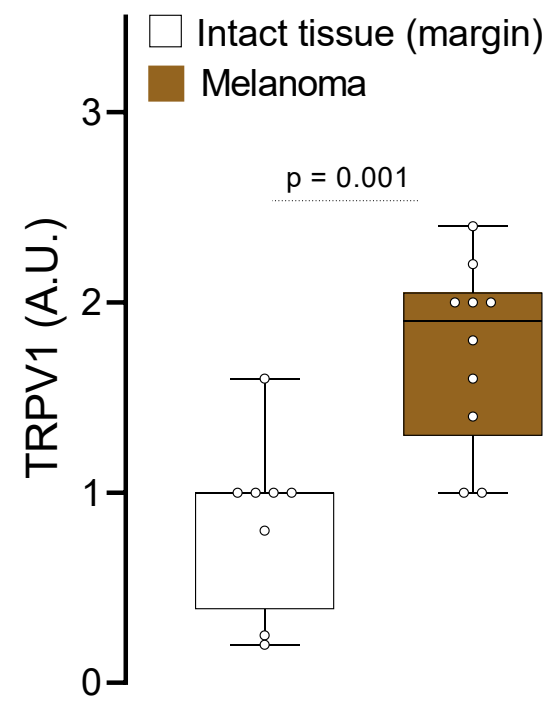

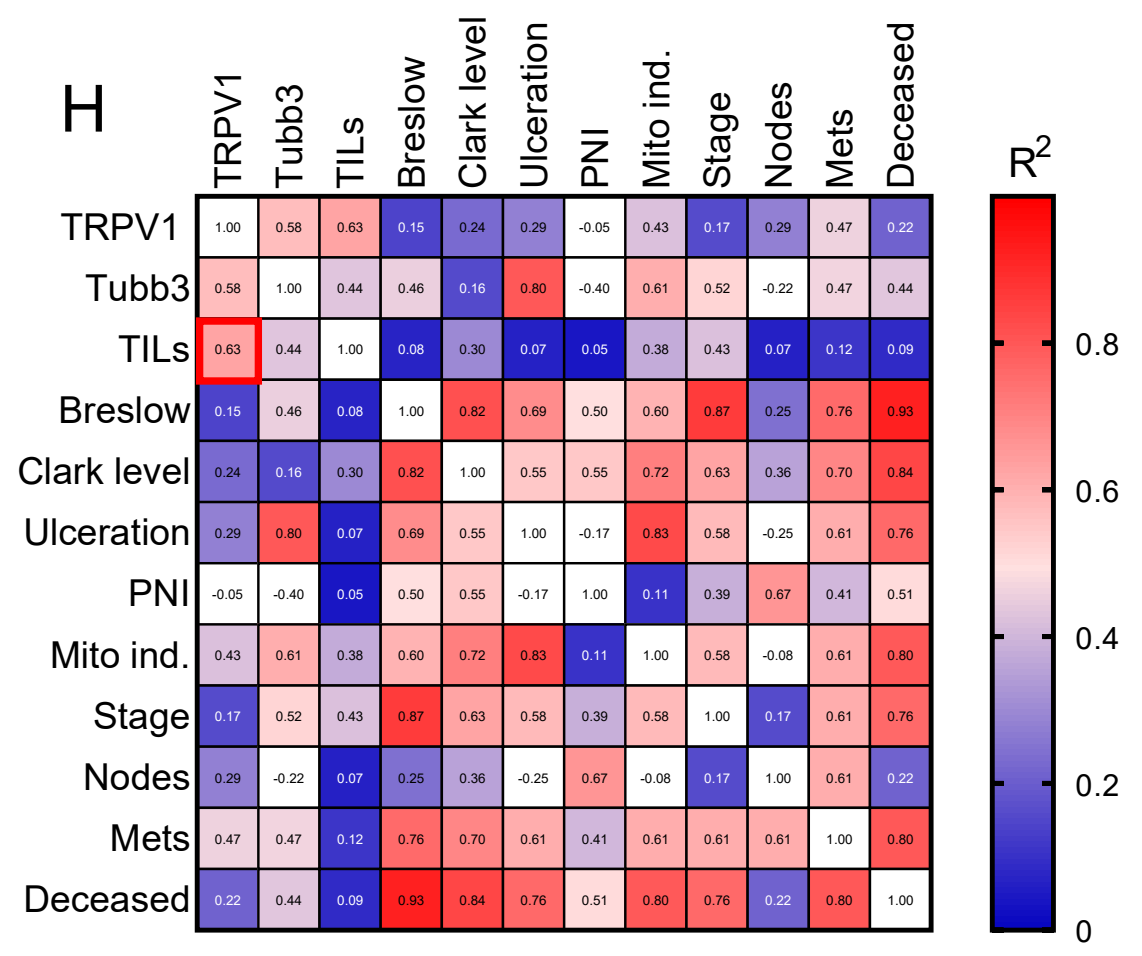

Supplementary Figure 1: TRPV1+ neurons innervate patients' melanoma. Patients' melanoma sections were stained with Hematoxylin Eosin (A-I), and the presence of TRPV1 (D-F; brown) neurons was analyzed by immunohistochemistry. Increased level of TRPV1 ${ }^{+}$cells $(\mathbf{J})$ were found in the tumor (delimitated by red square; A-B, D-E) compared to healthy skin (indicated by blue square; A, C, D, F). Increased TRPV1 immunolabeling in tumor sections primarily correlated with enhanced levels of tumor-infiltrating leukocytes $(\mathbf{G})$ as scored from a retrospective correlation analysis performed on the patient's pathology report $(\mathrm{n}=10$ patients). Data are shown as mean \pm S.E.M; unpaired Student $t$-test (G); $p$-values are shown in the figure. Scale = $100 \mu m(\boldsymbol{A}-\boldsymbol{F})$. Pearson correlation $\left(R^{2}\right)$ is shown as a heatmap $(\boldsymbol{H})$. 

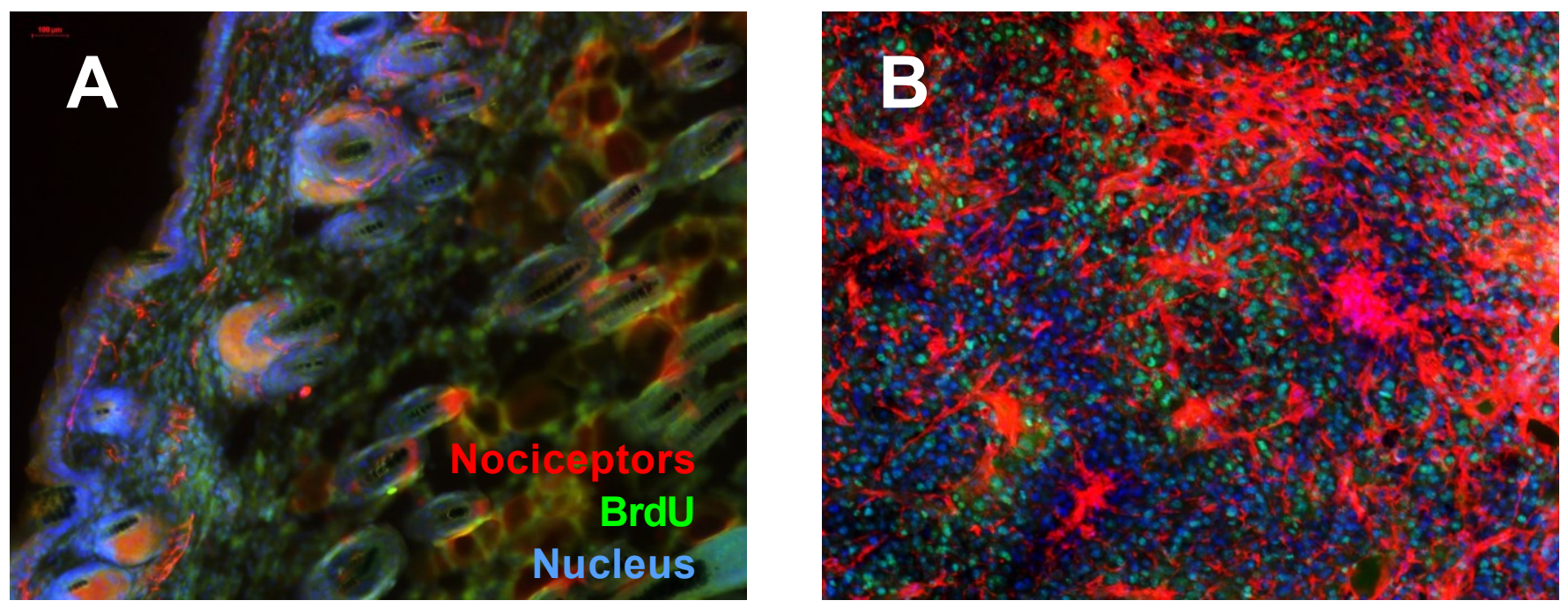

Supplementary Figure 2: TRPV1 ${ }^{+}$neurons innervate B16F10 tumors. Nociceptor neuron (TRPV1 cre::Td-tomato ${ }^{\mathrm{f} / \mathrm{wt} \text {; red) }}$ reporter mice hindpaw were inoculated $\left(1 \times 10^{5}\right.$ cells; i.d.) with non-tumorigenic keratinocytes (A) or B16F10 cancer cell (B). 14 days after inoculation, we found that TRPV1+ nerve fibers (red) densely innervated B16F10-inoculated skin (B; green; BrdU' labels proliferating cells) as compared to keratinocytes-injected skin (A). Scale $=100 \mu m(A-B) . T R P V 1^{+}$nociceptors are labeled in red, BrdU proliferating cells are labeled in green and nuclei are labeled in blue (A-B). 


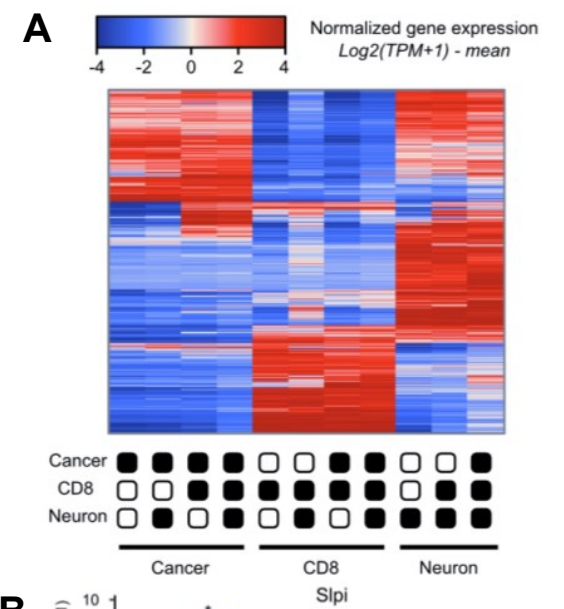

B
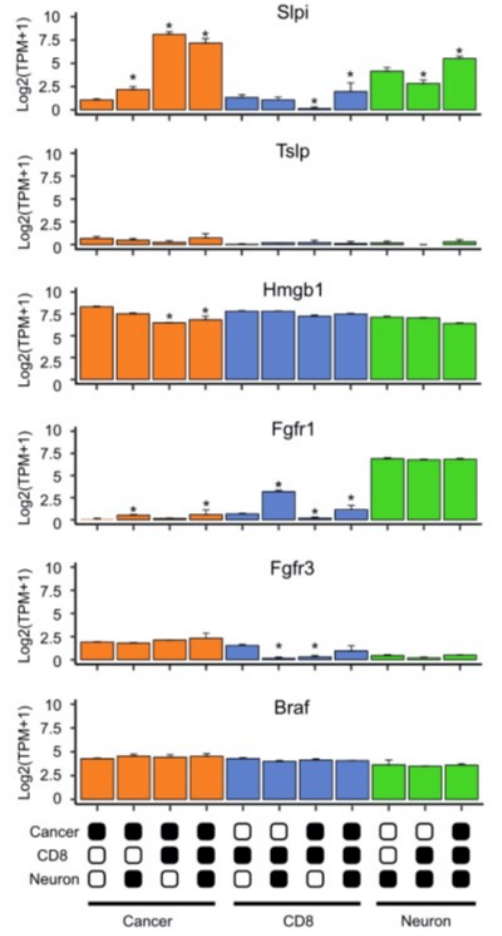

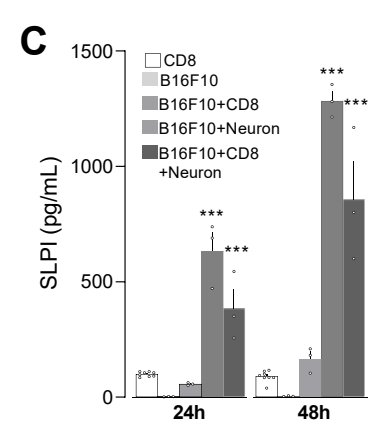

D

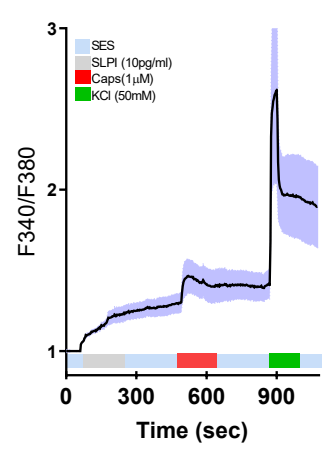

E

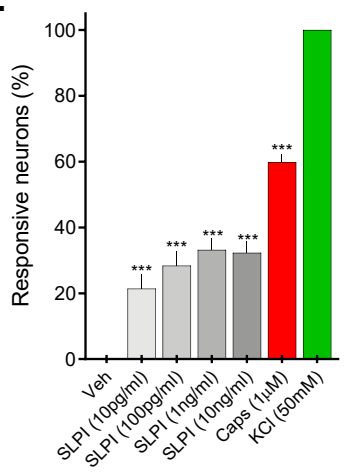

G
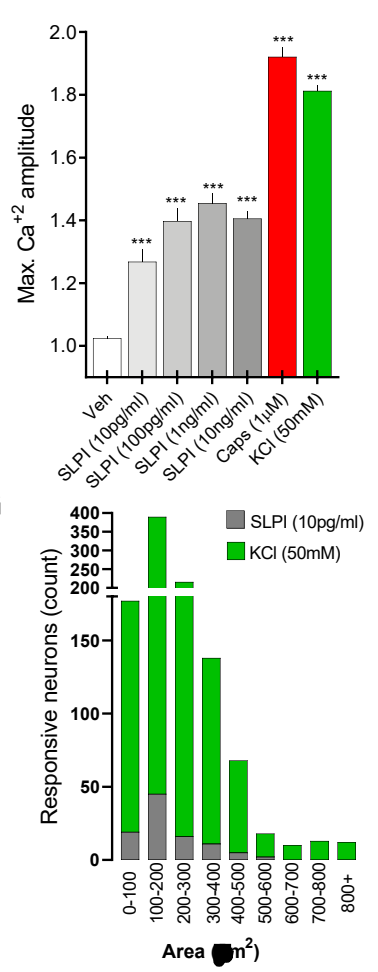

I

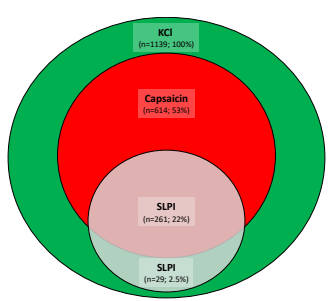

J

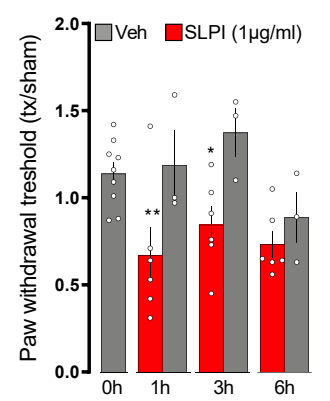

H

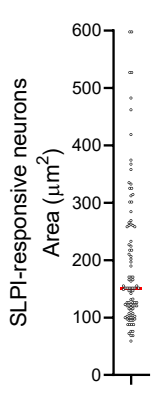

K

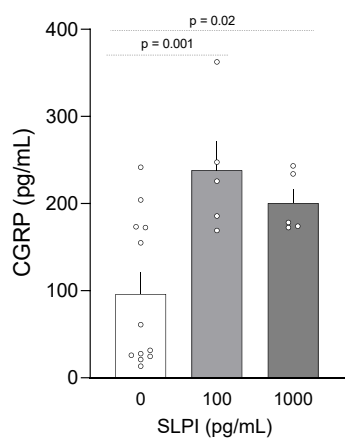

Supplementary Figure 3: Cancer-secreted SLPI sensitizes nociceptor neurons. Naive DRG neurons (TRPV1 ${ }^{\text {cre:::QuASR2- }}$ eGFPf/wt), B16F10-mCherry-OVA, and OVA-specific cytotoxic CD8+ T-cells were cultured alone or in combination. After 48h, the cells were harvested, FACS-purified, and RNA sequenced. Hierarchical cluster analysis was performed using normalized gene expressions from protein-coding genes (A). Differentially expressed genes (DEG) were calculated, and SLPI (Secretory Leukocyte Protease Inhibitor) was found to be overexpressed in cancer cells when co-cultured with OVA-specific cytotoxic $\mathrm{CD}^{+}$T-cells, DRG neurons or both populations. In the presence of protease inhibitors, B16F10-mCherry-OVA were cultured alone, with naive DRG neurons and/or with OVA-specific cytotoxic CD8 ${ }^{+}$T-cells. The culture supernatant was harvested after 24 or $48 \mathrm{~h}$, and SLPI secretion was measured by ELISAs. We found increased SLPI secretion by B16F10-mCherry-OVA when co-cultured with naive DRG neurons and OVA-specific cytotoxic CD8 ${ }^{+}$T-cell, with maximal effect after 48h (C). Next, we used calcium microscopy to probe whether SLPI directly activates cultured DRG neurons. We found that SLPI $(10-10000 \mathrm{ng} / \mathrm{ml})$ activated $\sim 20 \%$ of DRG neurons (D-F). Compatible with being nociceptor neurons, SLPI-responsive neurons are mostly smallto medium-sized neurons $\left(\mathbf{G}-\mathbf{H}\right.$; mean area $\left.=151 \mu \mathrm{m}^{2}\right)$ and largely capsaicin-responsive neurons $(\mathbf{I} ; \sim 42 \%)$. The right hindpaw of naive mice was injected with saline or SLPI $(1 \mu \mathrm{g} / 25 \mu \mathrm{L}$; i.d.), and the mice's noxious thermal nociceptive threshold was measured (0-6h). In contrast with the contralateral paw, the ipsilateral paw injected with SLPI showed thermal hypersensitivity. Saline had no impact on the mice's thermal sensitivity threshold (J). Naive DRG neurons were cultured and stimulated with SLPI $(0-1000 \mathrm{pg} / \mathrm{ml})$ for $3 \mathrm{~h}$. We found that SLPI (100-1000 pg/ml) triggered CGRP release from cultured naive DRG neurons (K). Data are shown as mean \pm S.D (B) or mean \pm S.E.M (C-H; J-K); one-way ANOVA post hoc Bonferroni (B, C, E, F); or unpaired Student $t$-test $(\mathbf{J}, \mathbf{K}) ; p$-values are shown in the figure. Hierarchical clustering of sorted neuron molecular profiles displays distinct groups of transcripts enriched in each group. Transcript per millions is shown as a heatmap $(\boldsymbol{A})$. 
A

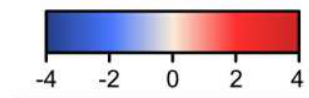

Normalized gene expression Log2(TPM+0.01) - mean

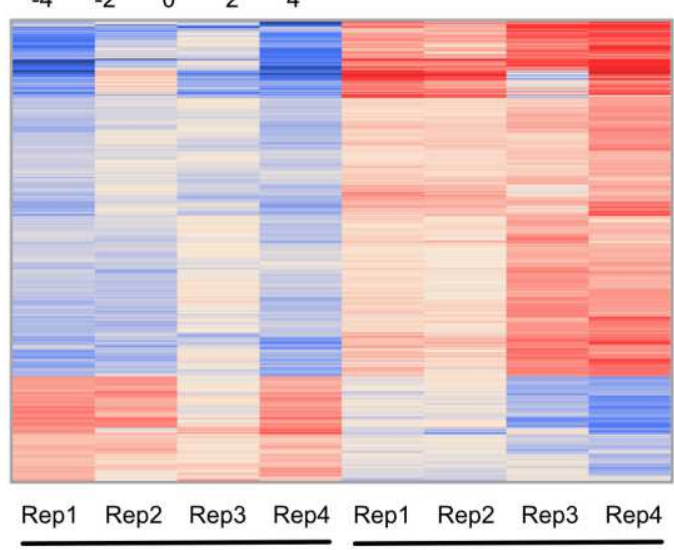

Nociceptors Tumor-exposed nociceptors
B

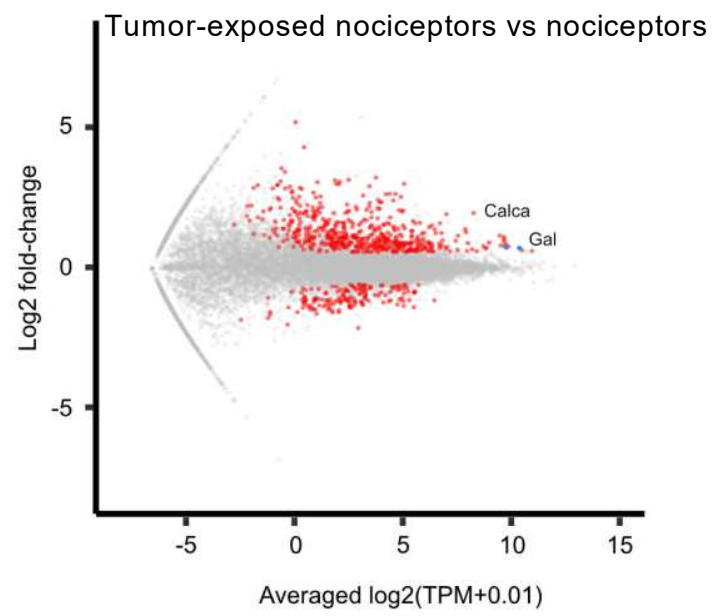

C

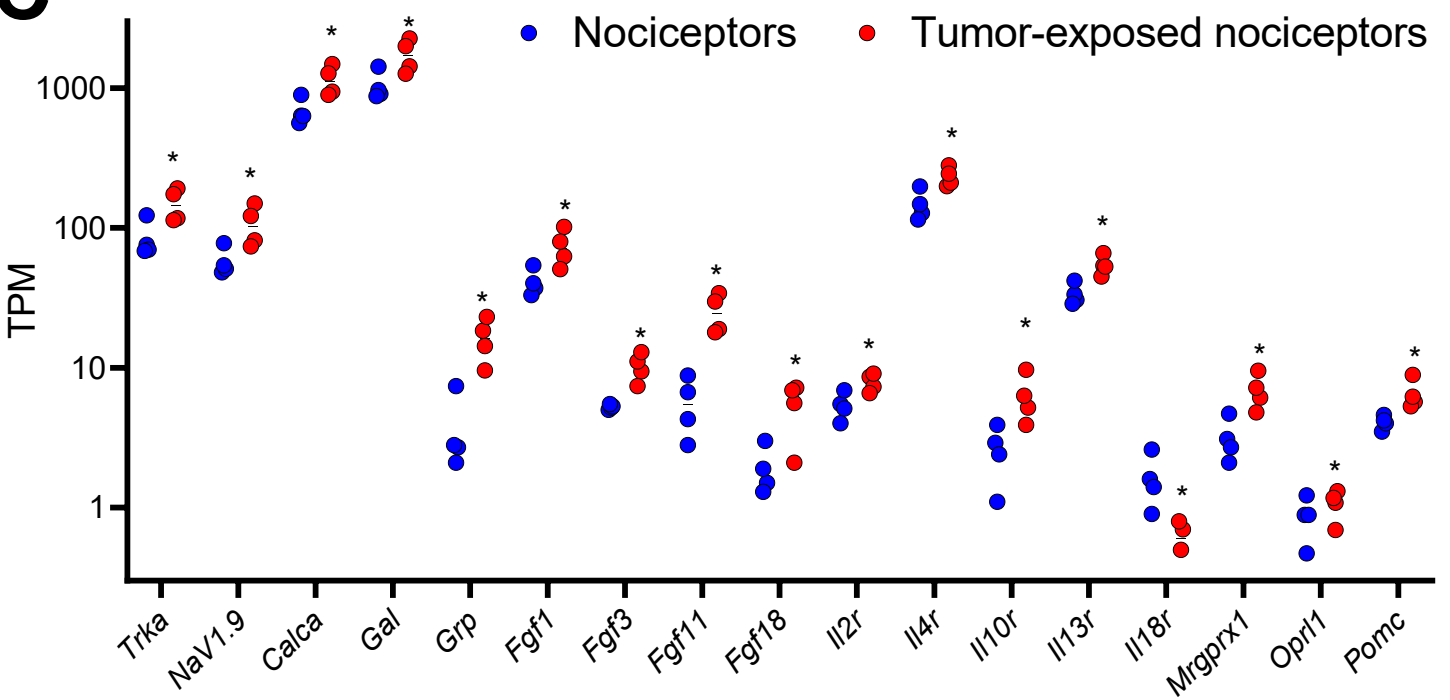

Supplementary Figure 4: DRG neurons overexpress CGRP when cultured with cancer cells. Naive DRG neurons (TRPV1 1 cre::QuASR2-eGFPf/wt) were cultured alone or in combination with B16F10-mCherry-OVA. After 48h, the cells were harvested, FACS-purified, and RNA sequenced. Differentially expressed genes (DEG) were calculated, and, among others, Trka and Calca were overexpressed in TRPV1 ${ }^{+}$(FACS-purified eGFP-expressing cells) neurons when co-cultured with B16F10-mCherry-OVA (A-C). Data are shown as mean \pm S.E.M. (A) Hierarchical clustering of sorted neuron DEG, show distinct groups of transcripts enriched in TRPV1+ neuron vs cancer-exposed TRPV1 ${ }^{+}$neuron populations (A). Row Z-score is shown as a heatmap $(\boldsymbol{A})$. Pairwise comparison of naive TRPV1+ neuron vs cancer-exposed TRPV1+ neuron populations showing differentially expressed transcripts as a volcano plot $(p<0.05 ; \boldsymbol{B})$. 
A

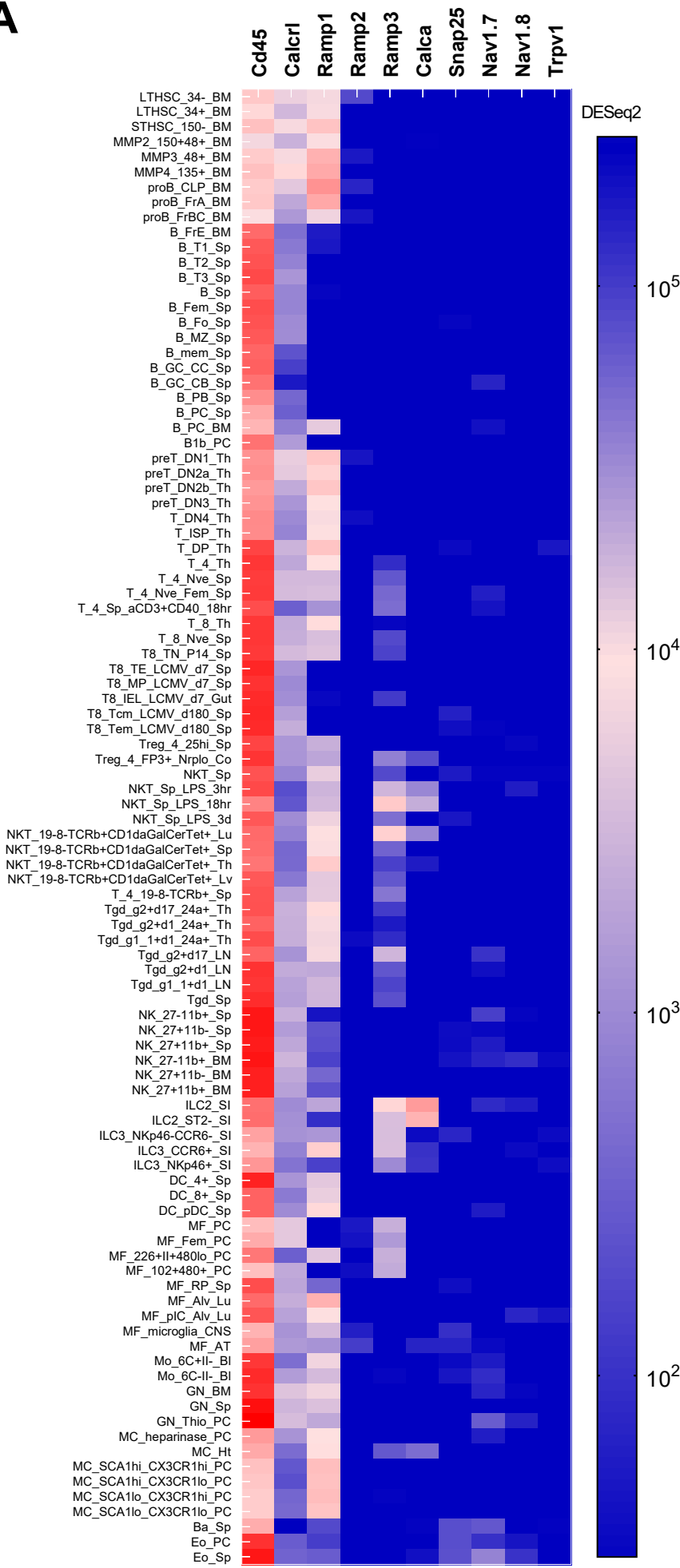

B

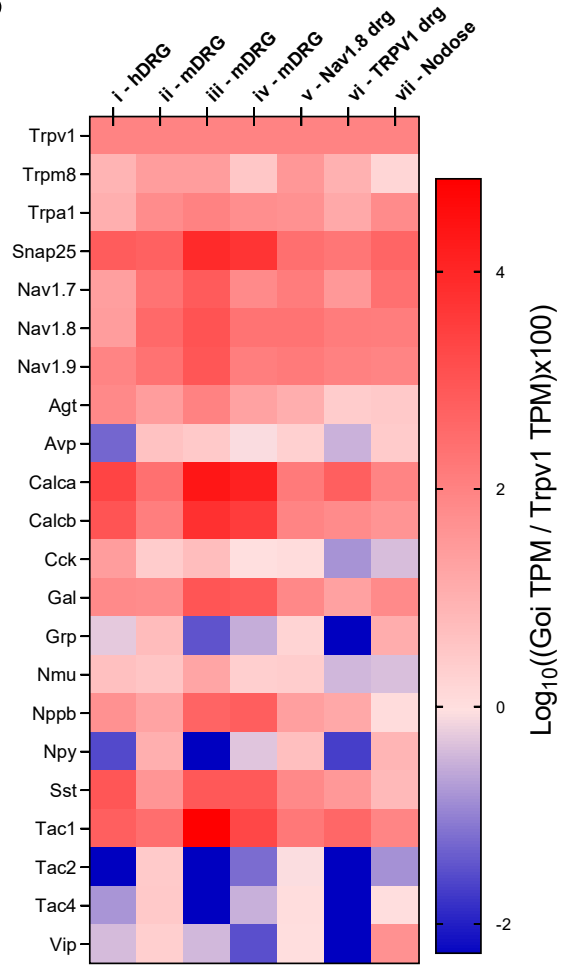

Supplementary Figure 5: Mouse immunocytes do not express TRPV1. ImmGen RNA sequencing of leukocyte subpopulations ${ }^{63}$ reveals their basal expression of Cd45 and Ramp1. In contrast, immune cells do not express Snap25, Trpv1, or Nav1.8 (A). Heatmaps data are shown as DESeq2 on a logarithmic scale (A). A meta-analysis of seven published nociceptor neuron expression profiling datasets ${ }^{64}$ revealed the basal expression of sensory neuron markers (Trpv1, Trpa1) and neuropeptides (Sp, Vip, Nmu, Calca; B). Expression across datasets was ratioed over Trpv1 and multiplied by 100. The Log 10 of these values is presented as a heatmap (B). i) RNA sequencing of human lumbar neurons ${ }^{65}$; ii) microarrays of mouse FACSsorted Nav1. $8^{+}$neurons ${ }^{66}$; iii) and iv) single-cell RNA sequencing of mouse lumbar neurons ${ }^{67,68 ;}$ v) microarray profiling of mouse whole DRG ${ }^{66}$; vi) performed RNA sequencing of mouse TRPV1+ neurons ${ }^{69}$; vii) single-cell RNA sequencing of mouse vagal ganglia ${ }^{70}$. 
A
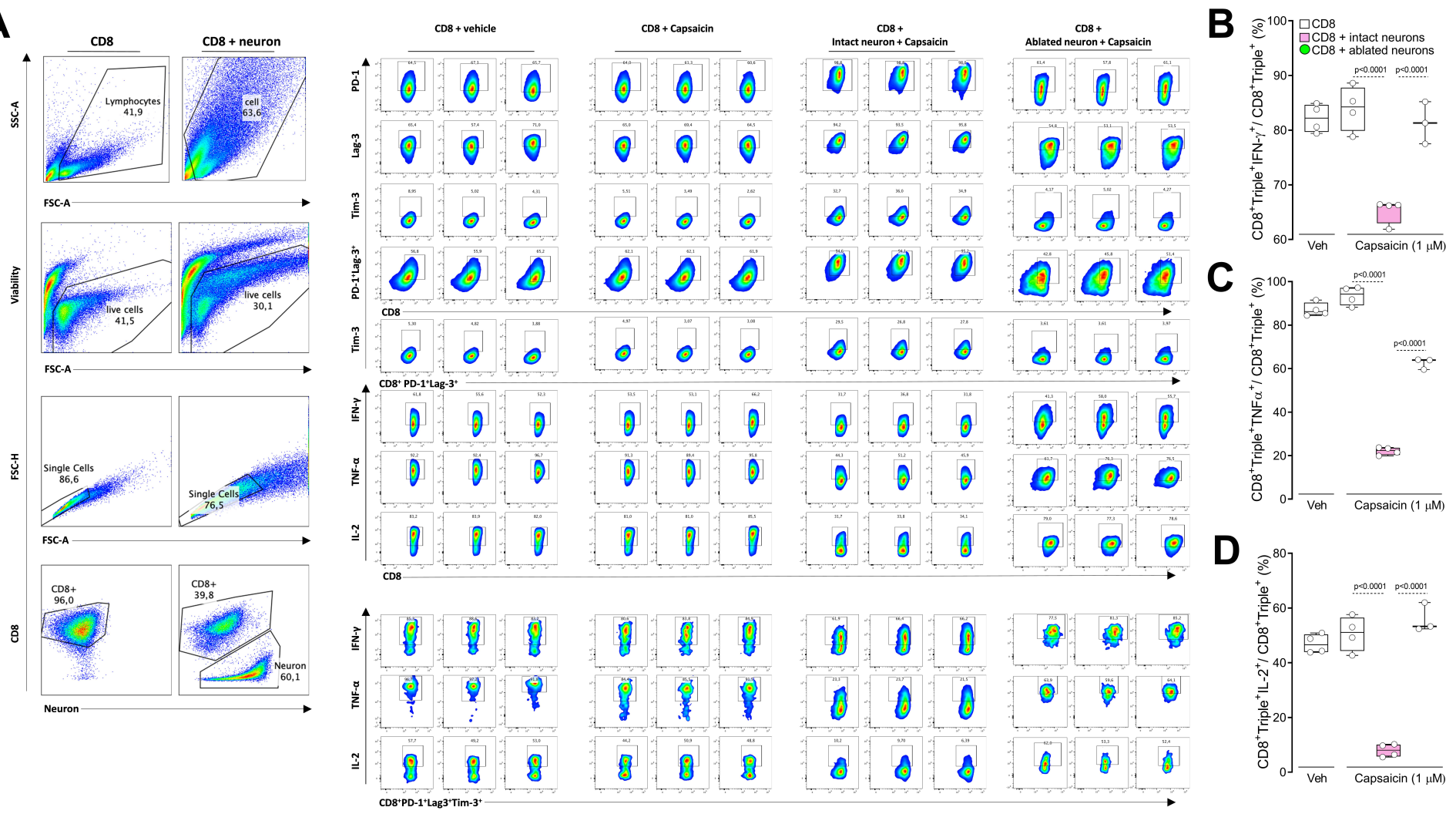

Supplementary Figure 6: Nociceptors drive $C D 8^{+}$T-cell exhaustion. Splenocytes-isolated CD8 ${ }^{+} \mathrm{T}$-cells were cultured under Tc1-stimulating conditions for $48 \mathrm{~h}$. The cells were then cultured or not with intact (TRPV1wt::DTA $\mathrm{A}^{\mathrm{fl} / \mathrm{wt})}$ or nociceptor ablated (TRPV1 $\left.{ }^{\text {cre:::DTA }}{ }^{\mathrm{fl} / \mathrm{wt}}\right) \mathrm{DRG}$ neurons and exposed to capsaicin $(1 \mu \mathrm{M}$, challenged once every two days) or its vehicle. As measured after 4 days stimulation, representative flow cytometry plots ( $n=3 /$ group) show that capsaicin-stimulated intact (TRPV1wt:::DTA $A^{\mathrm{f} / \mathrm{wt}}$ ) neuron increased the proportion of $\mathrm{PD} 1^{+} \mathrm{Lag}^{+} \mathrm{Tim}^{+}$cytotoxic CD8 ${ }^{+}$T-cells (A). Total (A) and $\mathrm{PD}^{+}$Lag3 $^{+} \mathrm{Tim}^{+}$(B-D; labeled as Triple ${ }^{+}$) cytotoxic CD8 ${ }^{+}$T-cells showed decrease expression of IFNy ${ }^{+}(\mathbf{B}), \mathrm{TNFa}^{+}(\mathbf{C})$, and $^{+}$ $\mathrm{IL2}^{+}$(D) when co-culture with capsaicin-stimulated intact neurons. Capsaicin had no effect on cytotoxic CD8 ${ }^{+}$T-cells when cultured alone or with nociceptor ablated (TRPV1 ${ }^{\text {cre:::DTA }}{ }^{\mathrm{fl} / \mathrm{wt}}$ ) neurons (A-D). Data are shown as mean \pm S.E.M; one-way ANOVA; $p$ values and $n$ are shown in the figure $(B-D)$. The representative gating strategy used in figure $2 A-B$ is shown (n=3/groups). 


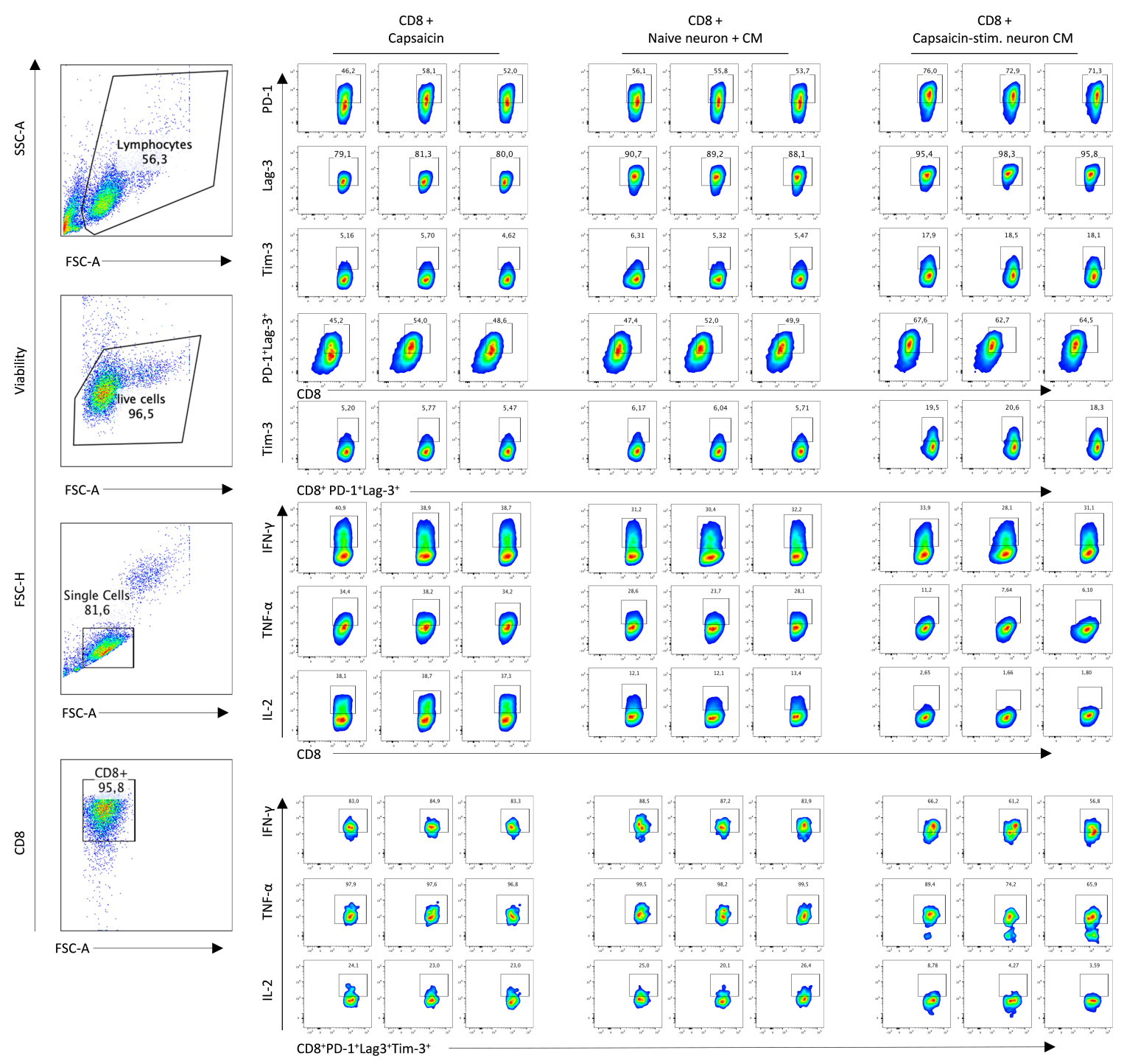

Supplementary Figure 7: Nociceptor neurons-released CGRP drive CD8 ${ }^{+}$T-cells exhaustion. Splenocytes-isolated CD8 ${ }^{+}$ T-cells were cultured under Tc1-stimulating conditions for $48 \mathrm{~h}$. In the presence of peptidase inhibitors $(1 \mu \mathrm{l} / 1000 \mu \mathrm{l})$, naive DRG neurons were cultured in the presence of BoNT/A $(100 \mathrm{pg} / 200 \mu \mathrm{l})$ or its vehicle for $24 \mathrm{~h}$. The cells were then washed, stimulated $(30 \mathrm{~min})$ with $\mathrm{KCl}(50 \mathrm{mM})$, and the conditioned media harvested. On alternate days for 4 days, the cytotoxic CD8 ${ }^{+} \mathrm{T}$-cells were exposed or not to a RAMP1 blocker (CGRP $\left.{ }_{8-37} ; 2 \mu \mathrm{g} / \mathrm{ml}\right)$ and challenge (1:2 dilution) with fresh $\mathrm{KCl}$-induced conditioned media from naive, or BoNT/A-silenced neurons. As measured after 4 days stimulation, representative flow cytometry plots ( $n=3 / g r o u p)$ show that $\mathrm{KCl}$-stimulated neuron conditioned media increase the proportion of PD1+ ${ }^{+} \mathrm{Lag} 3^{+} \mathrm{Tim}^{+}$cytotoxic $\mathrm{CD} 8^{+} \mathrm{T}$-cells. Such effect was absent when cytotoxic $\mathrm{CD}^{+} \mathrm{T}$-cells were co-exposed to the RAMP1 blocker or challenged with the neuron conditioned media harvested from BoNT/A-silenced neurons. The representative gating strategy used in figure 2C-D is shown ( $n=3$ /groups). 
A
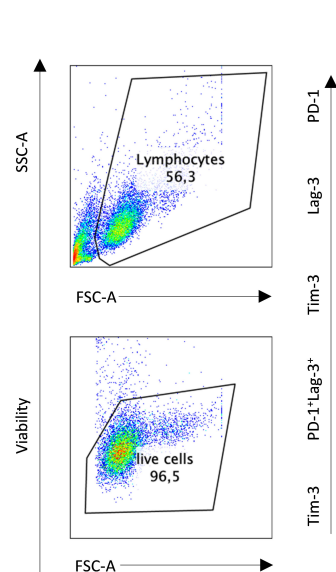

CD8

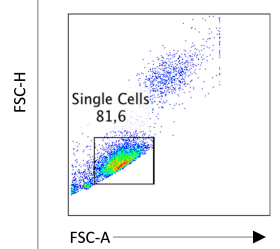

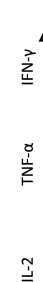

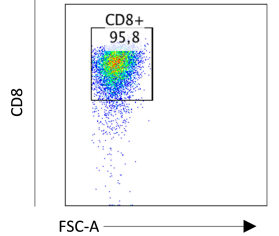

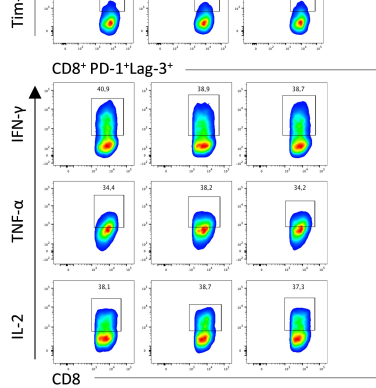

CD8+
Capsaicin

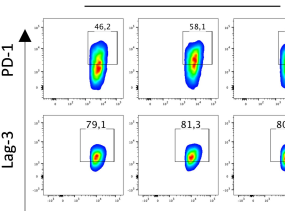

है 00

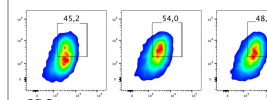

CD8

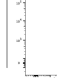

1月

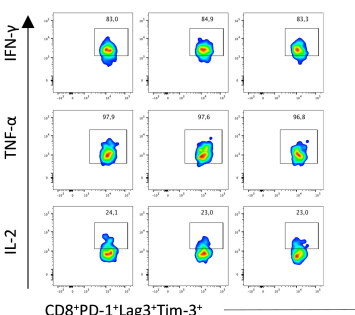

CD8 +

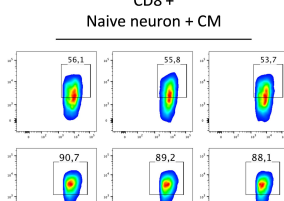

0 0.0

000

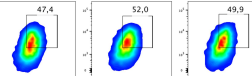

o.
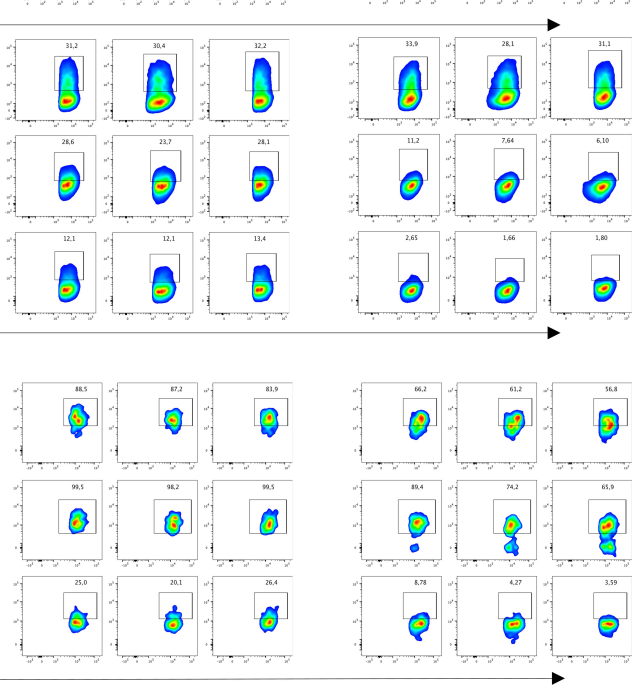

B

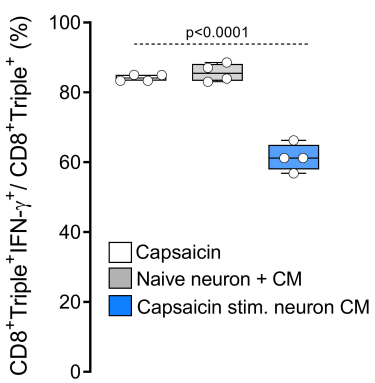

C

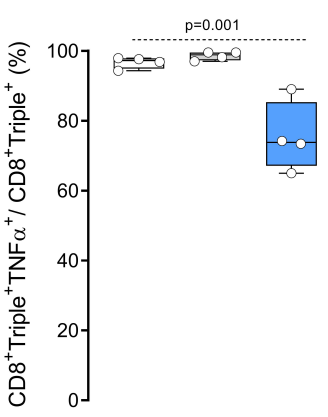

D

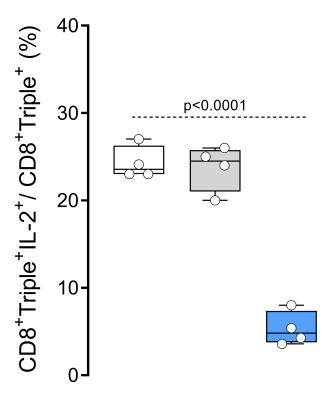

Supplementary Figure 8: Nociceptor neurons conditioned media drive CD8 ${ }^{+}$T-cells exhaustion. Splenocytes-isolated $\mathrm{CD}^{+} \mathrm{T}$-cells were cultured under Tc1-stimulating conditions for $48 \mathrm{~h}$. On alternate days for 4 days, the cytotoxic CD8 ${ }^{+} \mathrm{T}$-cells were co-exposed to IL-2 $(10 \mathrm{ng} / \mathrm{ml})$ and fresh conditioned media (1:2 dilution) harvested from capsaicin $(1 \mu \mathrm{M})$-stimulated DRG neurons, unstimulated neurons, or capsaicin. As measured after 4 days stimulation, representative flow cytometry plots $\left(n=3\right.$ /group) show that capsaicin-stimulated neuron conditioned media increase the proportion of PD1 ${ }^{+}$Lag $3^{+} \mathrm{Tim}^{+}{ }^{+}$cytotoxic CD8 ${ }^{+}$T-cells (A). Total (A) and PD1+ Lag3 $^{+} \mathrm{Tim}^{+}$(B-D labeled as Triple ${ }^{+}$) cytotoxic CD8 ${ }^{+}$T-cells showed decrease expression of $\mathrm{IFNY}^{+}(\mathbf{B}), \mathrm{TNFa}^{+}(\mathbf{C})$, and IL2 ${ }^{+}(\mathbf{D})$ when exposed to capsaicin-stimulated neuron conditioned media. Data are shown as mean \pm S.E.M; one-way ANOVA; $p$ values and $n$ are shown in the figure (B-D). 

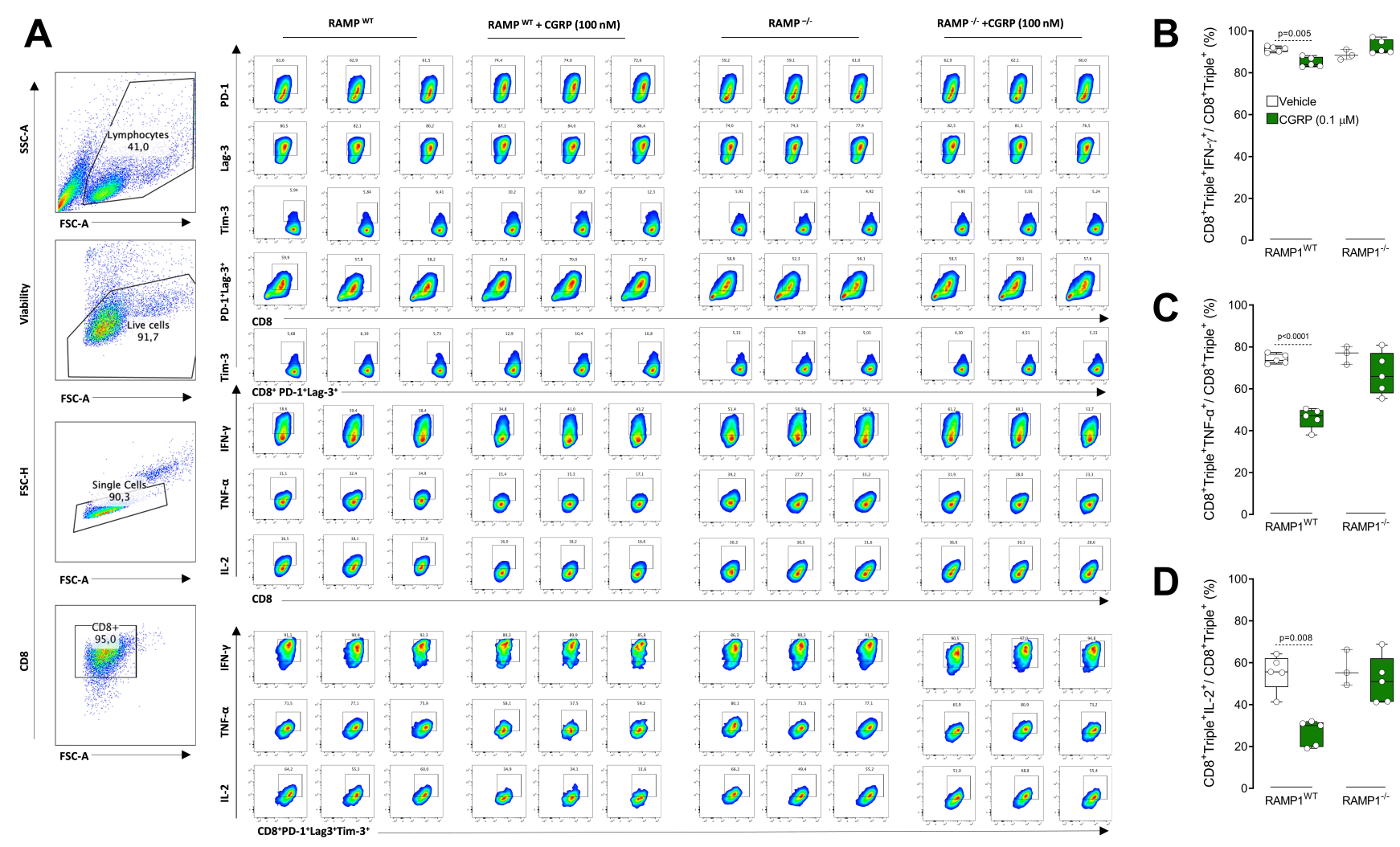

Supplementary Figure 9: CGRP drives $\mathrm{CD}^{+}{ }^{+}$-cells exhaustion. Splenocytes-isolated $\mathrm{CD} 8^{+} \mathrm{T}$-cells from wildtype and RAMP1-- mice were cultured under Tc1-stimulating conditions for $48 \mathrm{~h}$. On alternate days for 4 days, the cytotoxic CD8 ${ }^{+} \mathrm{T}$-cells were exposed CGRP $(0.1 \mu \mathrm{M})$ or its vehicle. As measured after 4 days stimulation, representative flow cytometry plots $\left(n=3 /\right.$ group) show that CGRP increased the proportion of PD1 $1^{+} \mathrm{Lag}^{+}{ }^{+} \mathrm{Tim} 3^{+}$in RAMP1 ${ }^{\text {wt }}$ cytotoxic CD8 ${ }^{+}$T-cells $(\mathbf{A})$. Total $(\mathbf{A})$ and PD1 ${ }^{+} \mathrm{Lag}^{+}{ }^{+} \mathrm{Tim}^{+}$(B-D labeled as Triple ${ }^{+}$) RAMP1 ${ }^{\text {wt }}$ cytotoxic CD8 ${ }^{+}$T-cells showed decrease expression of IFNy ${ }^{+}(\mathbf{B})$, $\mathrm{TNFa}^{+}(\mathbf{C})$, and IL2 ${ }^{+}(\mathbf{D})$ when exposed to CGRP. RAMP1-l- cytotoxic CD8 ${ }^{+}$T-cells were protected from the effect of CGRP (AD). Data are shown as mean \pm S.E.M; one-way ANOVA; $p$ values and $n$ are shown in the figure (B-D). The representative gating strategy used in figure $2 E-F$ is shown ( $n=3 /$ groups). 


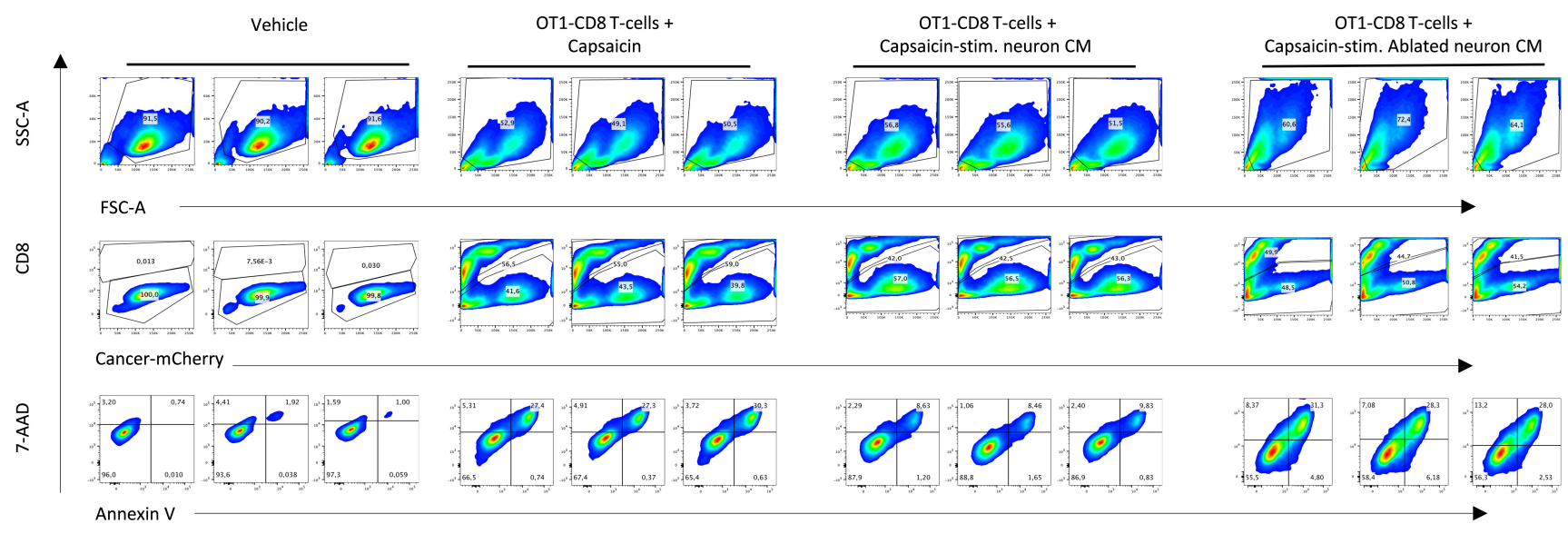

Supplementary Figure 10: Sensory neurons-released neuropeptides blunt cytotoxic T-cell anti-tumor immunity. Splenocytes-isolated CD8 ${ }^{+}$T-cells from naive OT-1 mice were cultured under Tc1-stimulating conditions for 48h. B16F10mCherry-OVA cells $\left(100,000\right.$ cells) were then cultured with or without OT- 1 cytotoxic CD8 ${ }^{+}$T-cells $(400,000$ cells). Once-daily for two consecutive days, the co-cultures were challenged (1:2 dilution) or not with fresh conditioned media harvested from capsaicin $(1 \mu \mathrm{M})$-stimulated intact (TRPV1 $\left.{ }^{\mathrm{wt}:: \text { DTA }}{ }^{\mathrm{fl} / \mathrm{wt}}\right)$ or nociceptor ablated (TRPV1 $\left.{ }^{\text {cre:::DTA }}{ }^{\mathrm{fl} / \mathrm{wt}}\right)$ DRG neurons. As measured after 48h exposure, Tc1-stimulated OT1-CD8 ${ }^{+}$T-cells lead to B16F10-OVA cell apoptosis (7-AAD+AnnexinV ${ }^{+}$), an effect reduced when exposed to capsaicin $(1 \mu \mathrm{M})$-stimulated neurons' conditioned media from intact DRG neurons. Capsaicin (1 $\mu \mathrm{M}$ )-stimulated nociceptor ablated (TRPV1 $\left.{ }^{\text {cre.::DTA }}{ }^{\mathrm{f} / \mathrm{wt}}\right)$ DRG neurons' conditioned media had no impact on OT1-CD8 ${ }^{+} \mathrm{T}_{\text {-cells }}$ elimination of B16F10-mCherry-OVA cells. The representative gating strategy used in figure $\mathbf{2 G}$ is shown ( $n=3 /$ groups). 


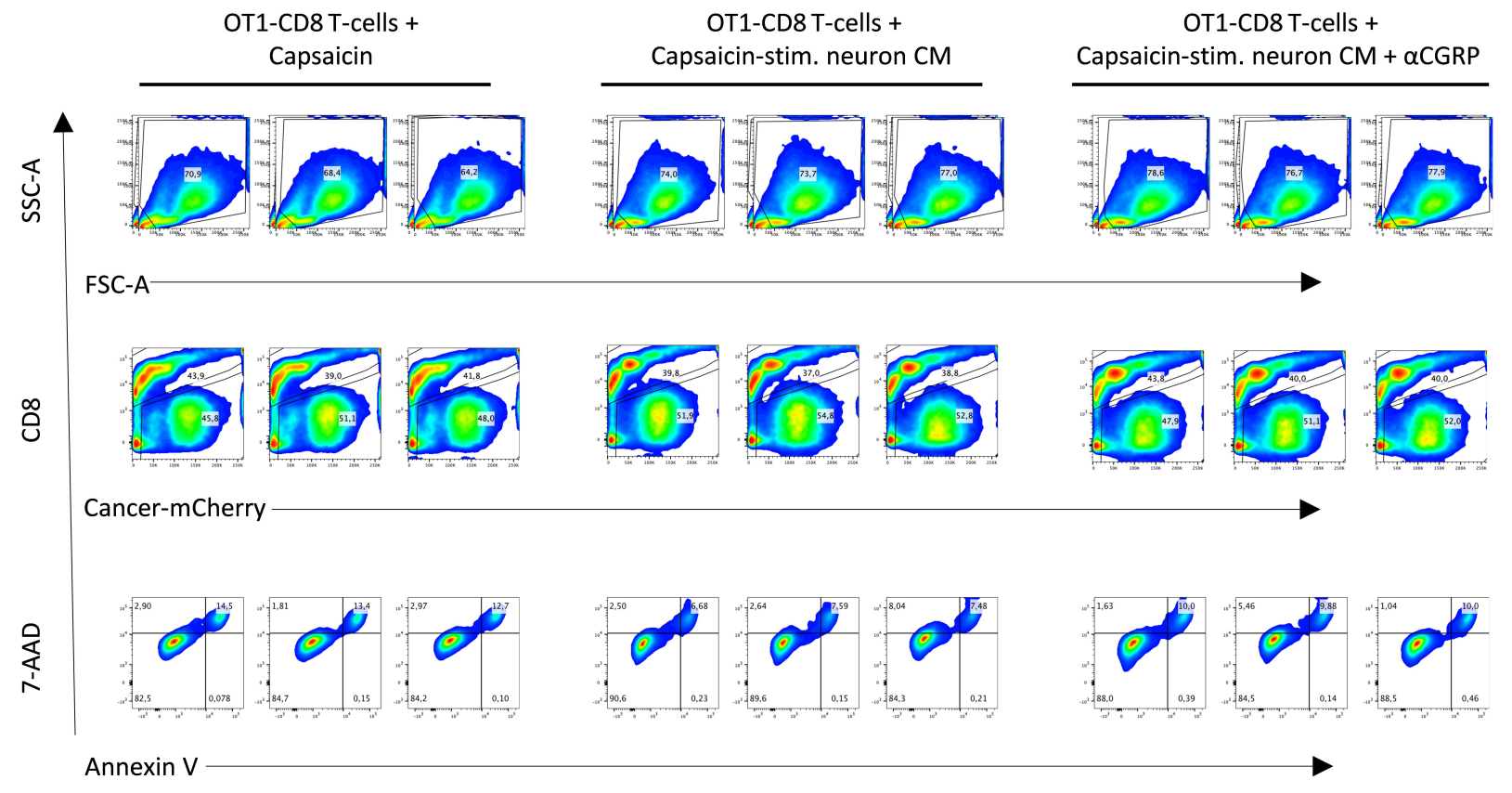

Supplementary Figure 11: Sensory neurons-released CGRP blunt cytotoxic T-cell anti-tumor immunity. Splenocytesisolated CD8 ${ }^{+}$T-cells from naive OT-1 mice were cultured under Tc1-stimulating conditions for 48h. B16F10-mCherry-OVA cells $\left(100,000\right.$ cells) were then cultured $(24 \mathrm{~h})$ with or without OT- 1 cytotoxic CD8 ${ }^{+}$T-cells $(400,000$ cells). The co-cultures were challenged (1:2 dilution; once) or not with fresh conditioned media harvested from $\mathrm{KCl}(50 \mathrm{mM})$-stimulated DRG neurons. As measured after $24 \mathrm{~h}$ exposure, $\mathrm{KCl}$-stimulated neurons' conditioned media reduced OT1-CD8 ${ }^{+} \mathrm{T}$-cells elimination of B16F10mCherry-OVA cells (7-AAD+Annexin $\left.\mathrm{V}^{+}\right)$, an effect decreased when the co-culture was co-exposed to the RAMP1 blocker CGRP $_{8-37}(2 \mu \mathrm{g} / \mathrm{ml})$. The representative gating strategy used in figure $2 \mathrm{H}$ is shown ( $n=3 /$ groups). 


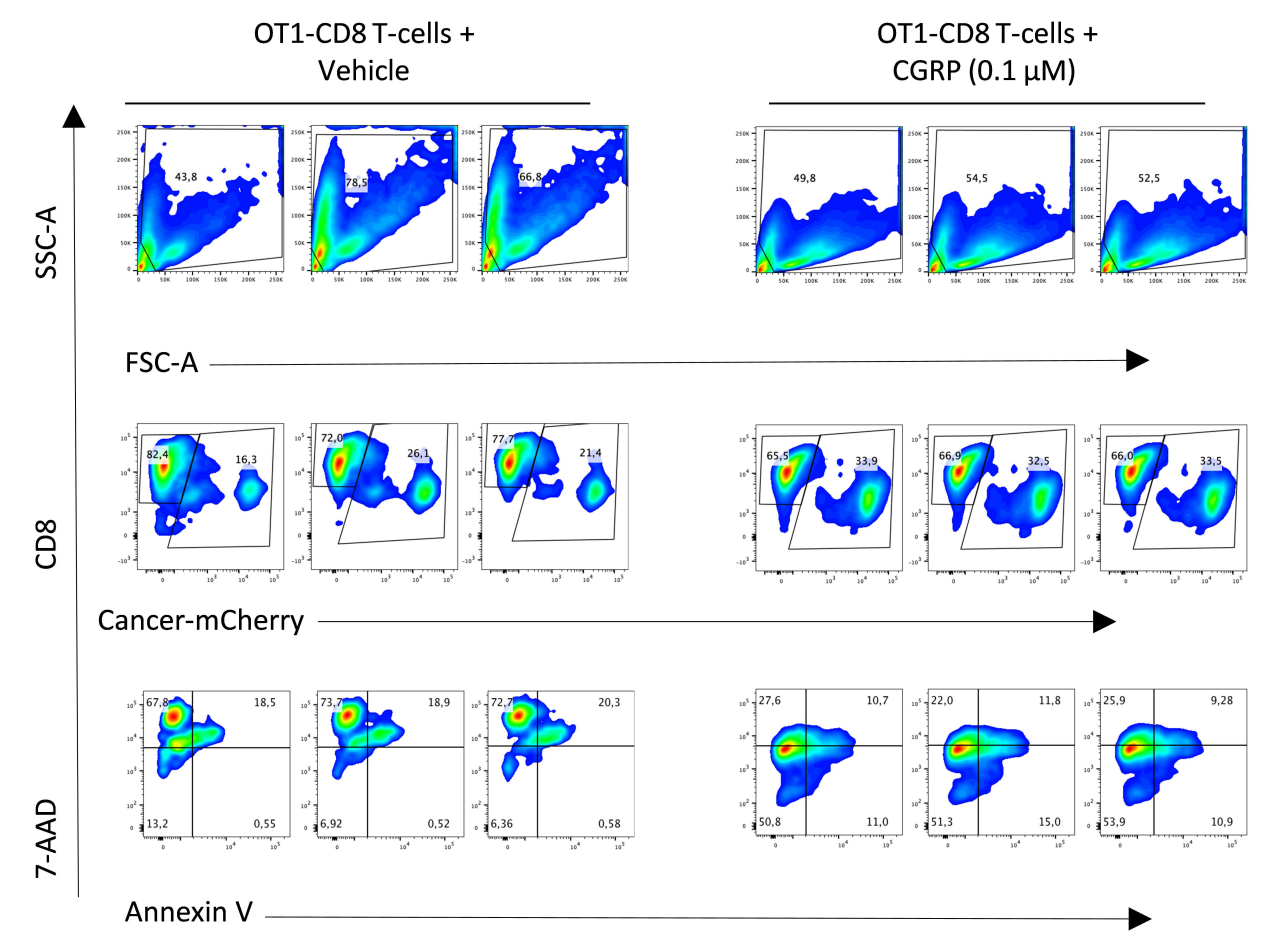

Supplementary Figure 12: CGRP blunt cytotoxic T-cell anti-tumor immunity. Splenocytes-isolated CD8 ${ }^{+} \mathrm{T}$-cells from naive OT-1 mice were cultured under Tc1-stimulating conditions for 48h. B16F10-mCherry-OVA cells $(100,000$ cells) were then cultured (24h) with or without OT-1 cytotoxic CD8 ${ }^{+}$T-cells (400,000 cells). As measured after 24h exposure, Tc1-stimulated OT1-CD8 ${ }^{+}$T-cells lead to B16F10-OVA cell apoptosis $\left(7-\mathrm{AAD}^{+} A n n e x i n \mathrm{~V}^{+}\right)$, an effect reduced when the co-culture was exposed to CGRP $(0.1 \mu \mathrm{M})$. The representative gating strategy used in figure $2 \mathrm{I}$ is shown ( $n=3 /$ groups). 
A
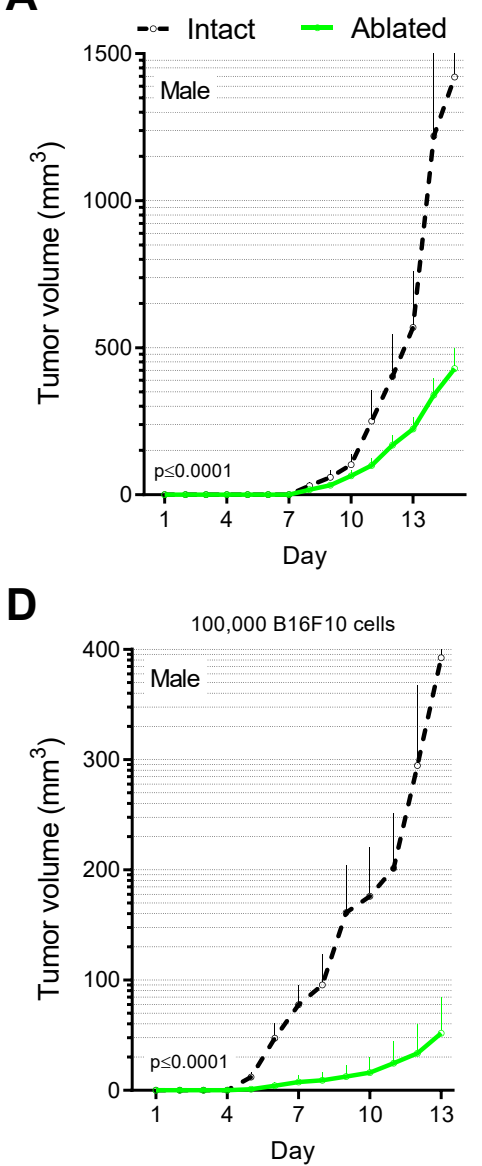

G

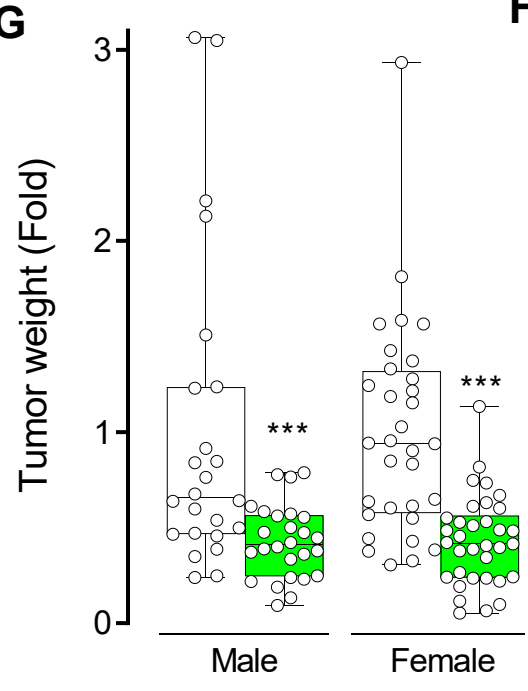

B

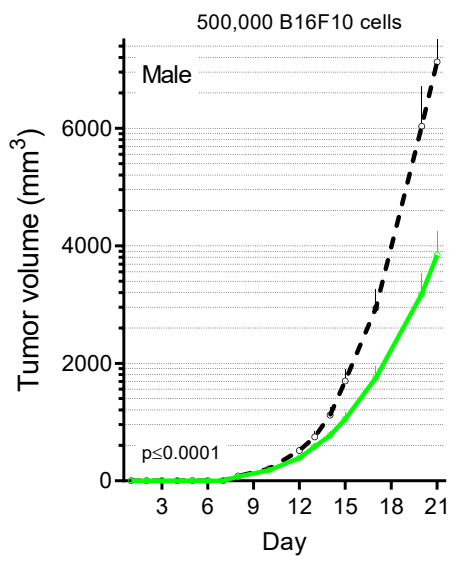

E

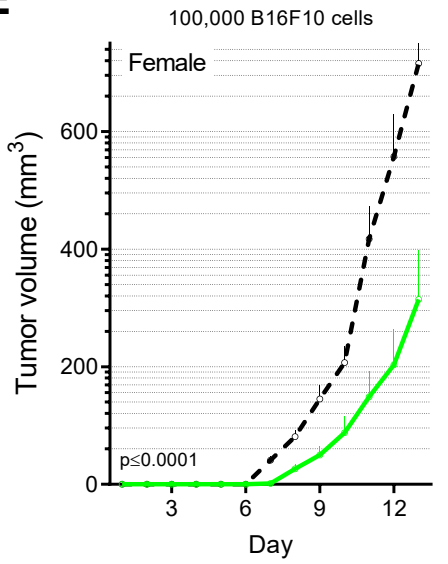

H

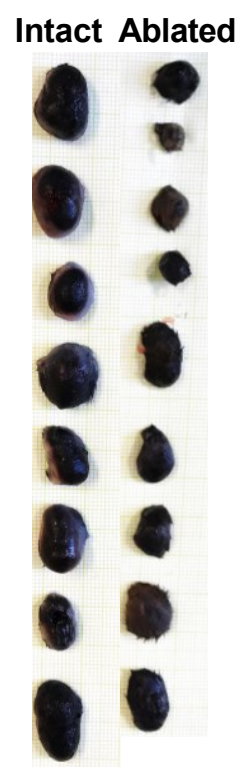

C

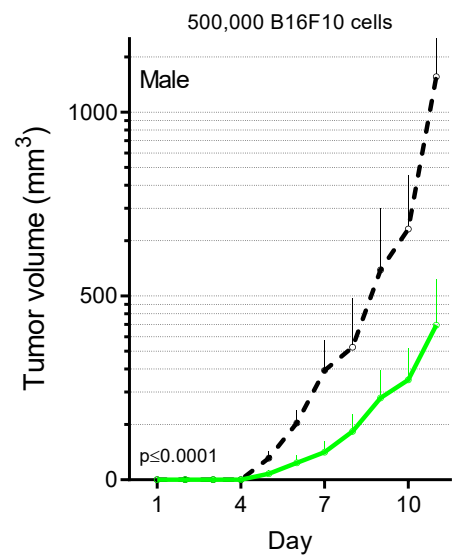

$\mathbf{F}$

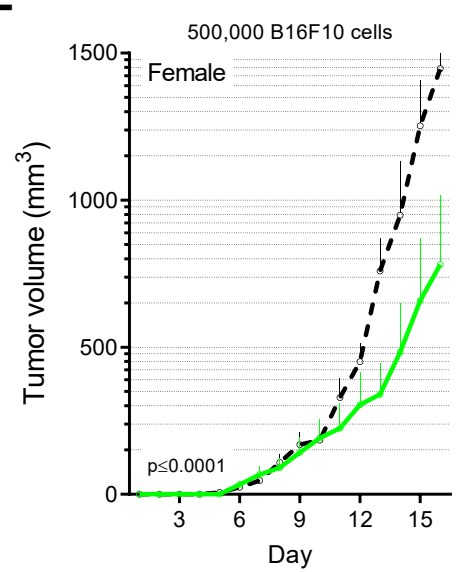

I

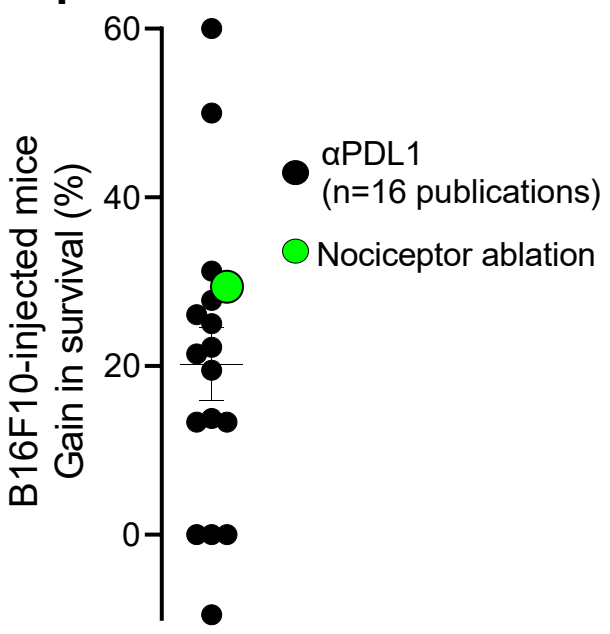

Supplementary Figure 13: Ablation of nociceptors decreases tumor growth. Orthotopic B16F10-OVA-mCherry cells $\left(1 \times 10^{5}-5 \times 10^{5}\right.$ cells; i.d.) were inoculated into 8-week-old male and female sensory neuron intact (TRPV1wt:.DTA $\left.A^{\mathrm{fl} / \mathrm{wt}}\right)$ or ablated (TRPV1 1 re:::DTA $\left.{ }^{\mathrm{fl} / \mathrm{wt}}\right)$ mice, and tumor weight, size, and growth were assessed. B16F10 growth (A-F; timing indicated in the figure), weight (G; measured on day 15$)$, and size (H; measured on day 15) were lower in mice whose nociceptors were genetically ablated (TRPV1 1 cre::DTA ${ }^{\mathrm{fl} / \mathrm{wt}}$ ). The median length of survival (retrospectively set as volume $\geq 1000 \mathrm{~mm}^{3}$ or when reaching other ethical endpoints) of B16F10-inoculated sensory neuron-ablated mice increased by $23 \%$, while that procured by aPDL1 blockade averaged $15.3 \%$ (I; a meta-analysis of 16 published studies). Data are shown as mean \pm S.E.M; two-way ANOVA post hoc Bonferroni (A-F), unpaired Student $t$-test (G); $p$-values are shown in the figure. 

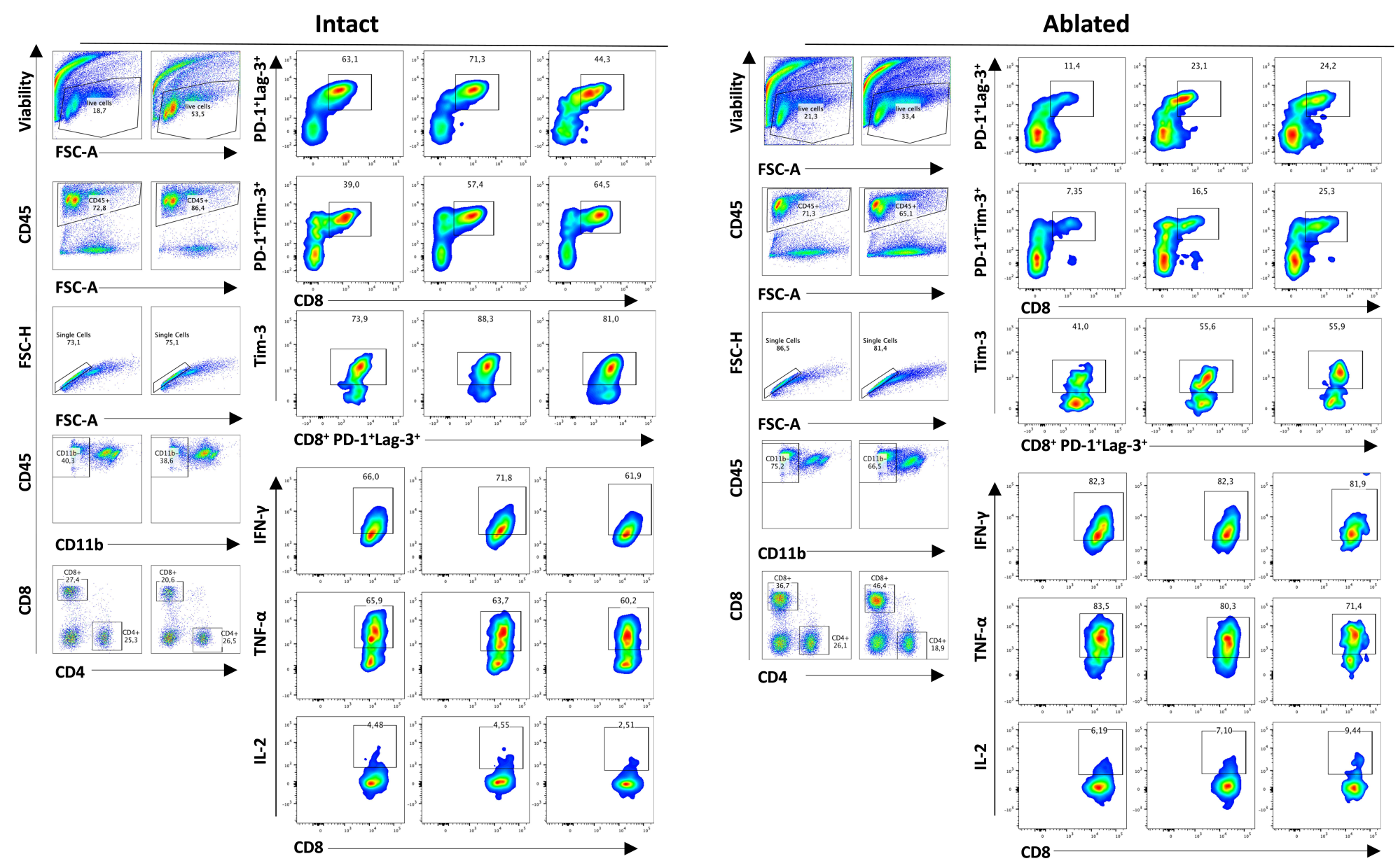

Supplementary Figure 14: Nociceptor ablation prevents exhaustion of intra-tumoral CD8 ${ }^{+}$T-cells. Orthotopic B16F10mCherry-OVA cells (500,000 cells; i.d.) were inoculated into 8-week-old male and female sensory neuron intact (TRPV1 wt:::DTA $^{\mathrm{f} / \mathrm{wt}}$ ) or ablated (TRPV1 ${ }^{\text {cre:::DTA }}{ }^{\mathrm{f} / \mathrm{wt}}$ ) mice. The impact of nociceptor neuron ablation on tumor-infiltrating CD8 ${ }^{+} \mathrm{T}^{-}$ cell exhaustion was immunophenotyped by flow cytometry fifteen days post-tumor inoculation. The representative gating strategy used in figure $3 C-E$ is shown ( $n=3 /$ groups). 
A

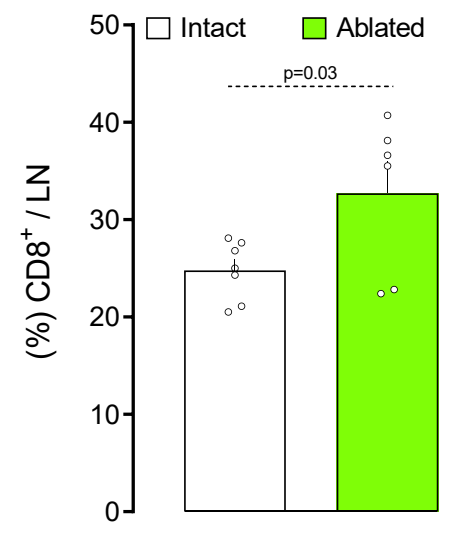

B

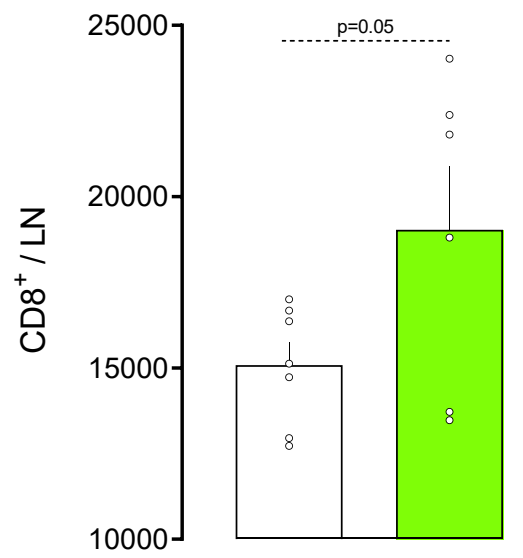

C

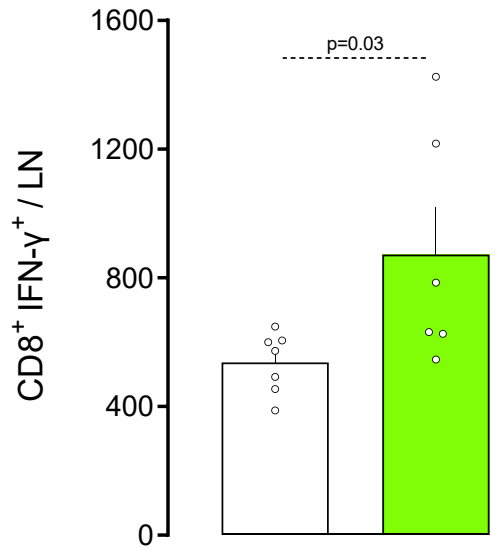

Supplementary Figure 15: Sensory neuron ablation increases activation of tumor-draining lymph nodes CD8 ${ }^{+}$T-cells. $^{-}$ Orthotopic B16F10-mCherry-OVA (500,000 cells; i.d.) were inoculated into 8-week-old male and female sensory neuron intact (TRPV1 ${ }^{\mathrm{wt} .:}: \mathrm{DT} \mathrm{A}^{\mathrm{f} / \mathrm{wt}}$ ) or ablated (TRPV1 ${ }^{\mathrm{cre}}:: \mathrm{DT} \mathrm{A}^{\mathrm{ff} / \mathrm{wt}}$ ) mice, and the number, relative exhaustion, and activation of tumor-draining lymph node leukocytes were immunophenotyped by flow cytometry. Fifteen days post-tumor inoculation, we found that nociceptor-ablated animals had an increased proportion $(\mathbf{A})$ and numbers $(\mathbf{B})$ of total $\mathrm{CD}^{+} \mathrm{T}$-cells as well as the number of IFNy ${ }^{+} \mathrm{CD}^{+}$T-cells (C) in tumor-draining lymph nodes. Data are shown as mean \pm S.E.M; unpaired Student $t$-test; $p$-values are shown in the figure. 

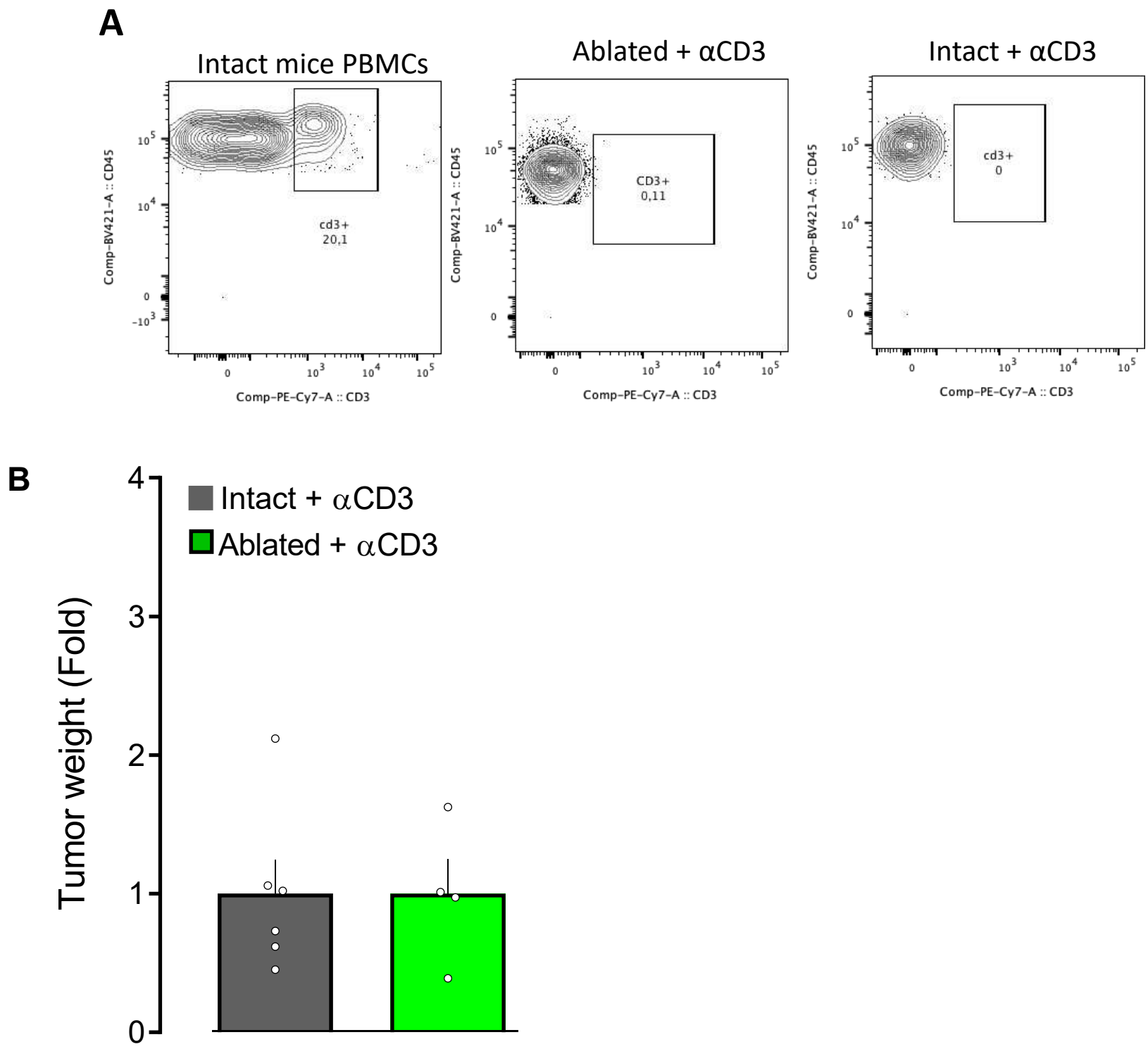

Supplementary Figure 16: Nociceptor neurons control CD3 anti-tumor immunity. Orthotopic B16F10-mCherry-OVA cells $(500,000$ cells; i.d.) were inoculated into 8-week-old male sensory neuron intact (TRPV1wt.:DTA fl/wt) or ablated (TRPV1 ${ }^{\text {cre:::DTA }}{ }^{\mathrm{fl} / \mathrm{wt}}$ ) mice. Systemic CD3 depletion ( $\alpha \mathrm{CD} 3,200 \mu \mathrm{g} /$ mouse; i.p.) was initiated 3 days before tumor inoculation and repeated every 3 days. Fifteen days post-tumor inoculation, we found that aCD3 treatment reduced the number of bloodcirculating $\mathrm{CD}^{+}$cells $\left(\mathbf{A} ; \mathrm{CD} 45^{+} \mathrm{CD}^{+}\right)$and rescued B16F10-mCherry-OVA tumor growth in nociceptor-ablated mice (B). Data are shown as mean \pm S.E.M. The tumor growth curve associated is shown in figure $3 F$. 


\section{A Yummer1.7}

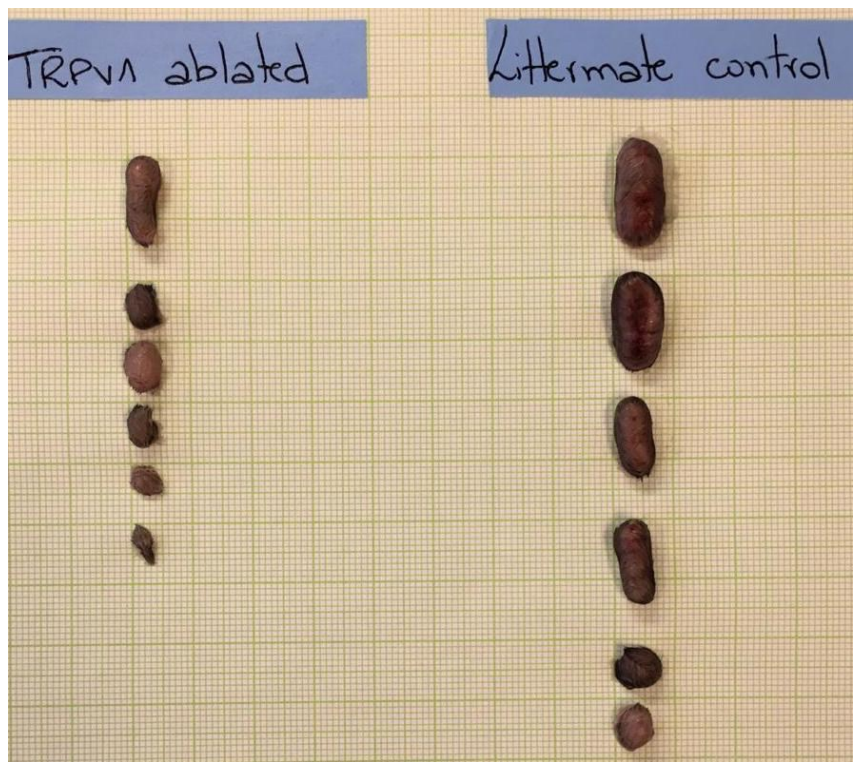

B

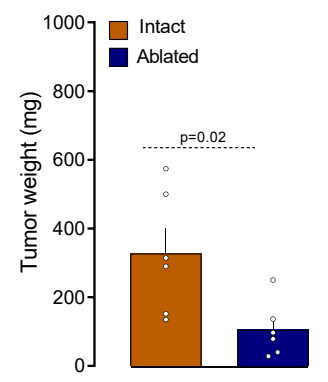

C

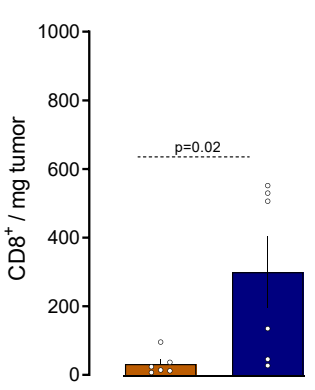

D

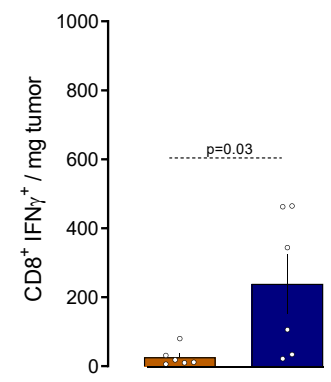

$\mathbf{E}$

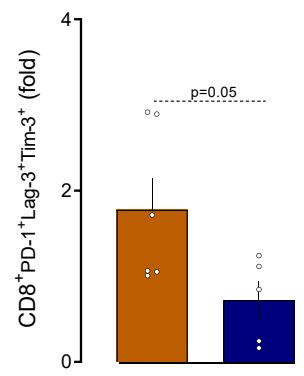

$\mathbf{F}$

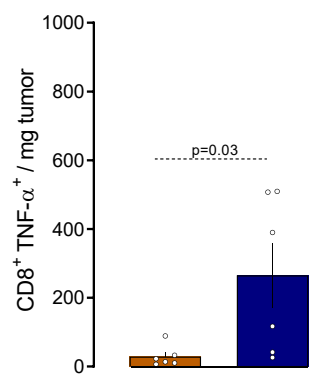

Supplementary Figure 17: Sensory neuron ablation decreases CD8 ${ }^{+}$T-cell exhaustion in YUMMER1.7-inoculated mice. Orthotopic YUMMER1.7 cells (500,000 cells; i.d.) were inoculated into 8-week-old male and female sensory neuron intact

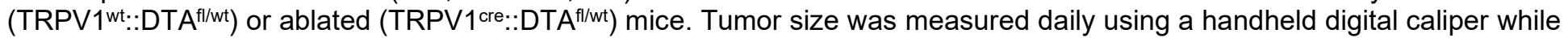
infiltrating $\mathrm{CD}^{+}{ }^{+}$T-cells' number and relative exhaustion were immunophenotyped by flow cytometry. Thirteen days post-tumor inoculation, we found that nociceptor-ablated animals had lower tumor volume $(\mathbf{A})$ and weight $(\mathbf{B})$ as well as a smaller proportion of $\mathrm{PD}^{+}{ }^{+} \mathrm{Lag}^{+} \mathrm{Tim3}^{+}(\mathbf{E}) \mathrm{CD}^{+} \mathrm{T}$-cells. In contrast, we discovered that sensory neuron ablation increased the intra-tumoral number of total (C), IFNy ${ }^{+}(\mathbf{D})$, and TNFa ${ }^{+}(\mathbf{F}) \mathrm{CD}^{+}$T-cells. Data are shown as mean \pm S.E.M; unpaired Student $t$-test (B-F); $p$-values are shown in the figure. The tumor growth curve associated is shown in figure $\mathbf{3 G}$. 


\section{A $\quad$ YUMM1.7}
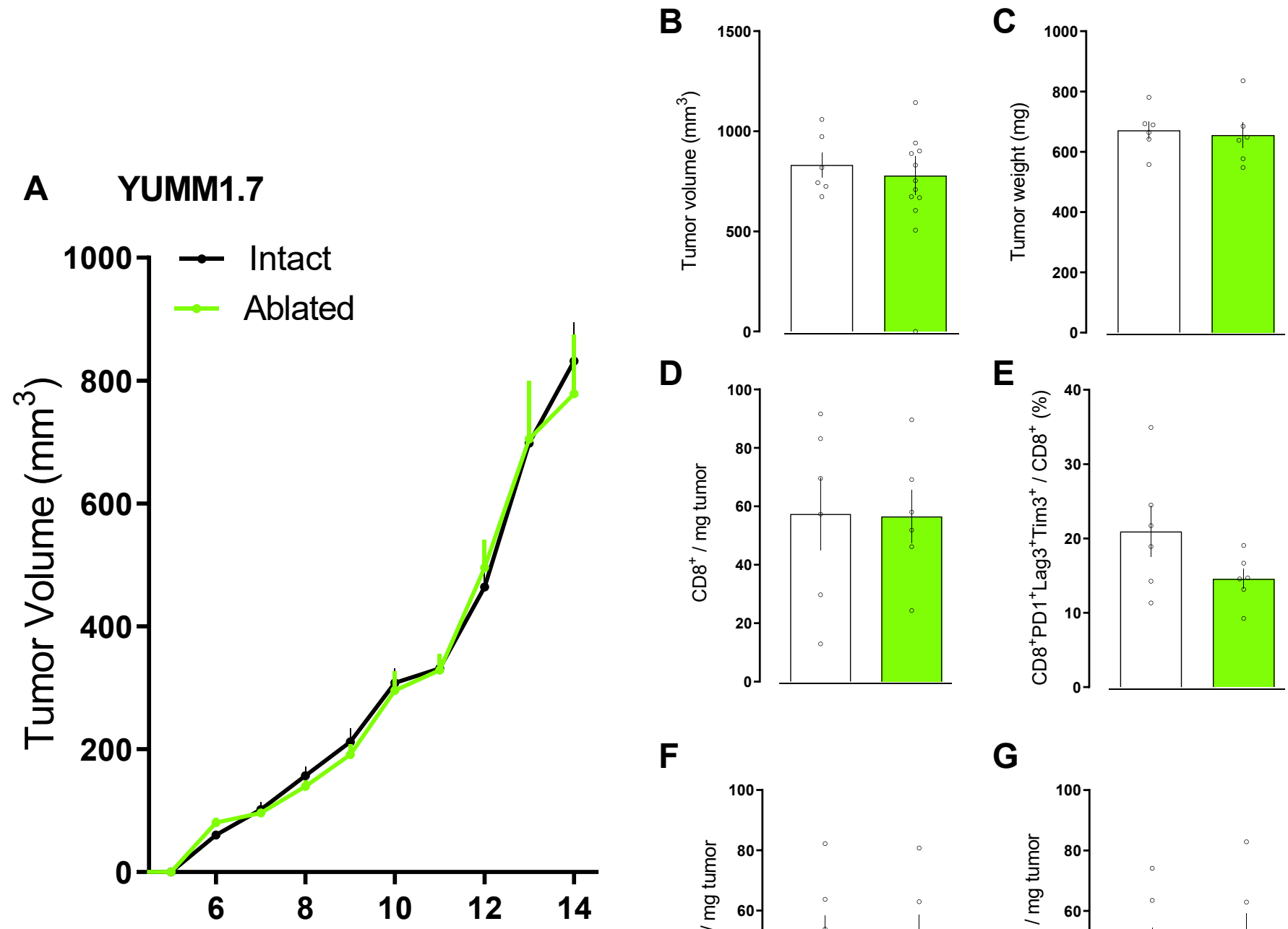

D

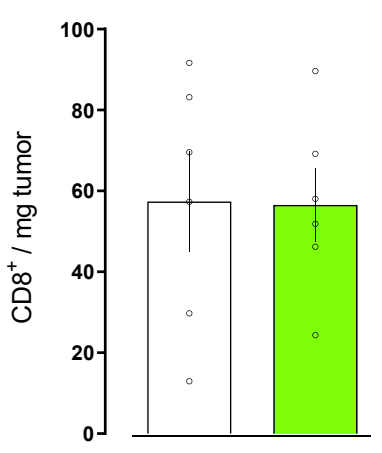

$\mathbf{F}$

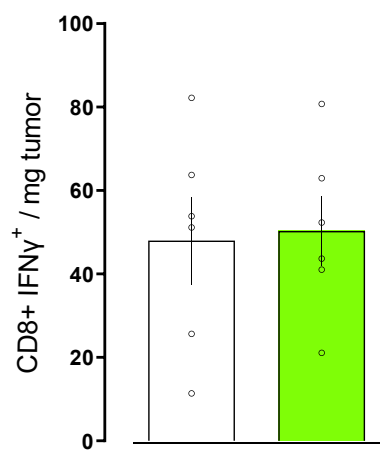

$\mathbf{E}$

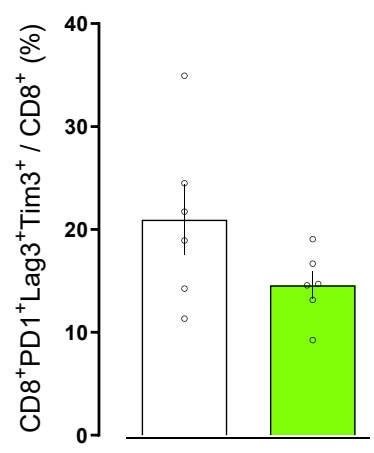

G

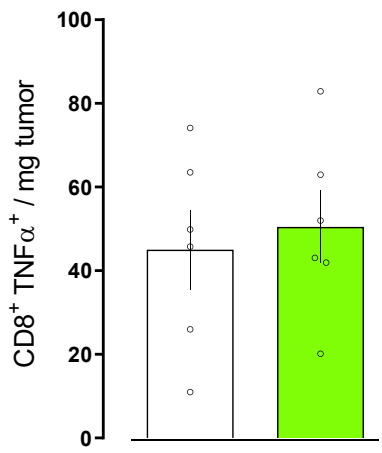

Supplementary Figure 18: Sensory neuron silencing does not impact YUMM1.7 tumor growth. Orthotopic YUMM1.7 cells (500,000 cells; i.d.), a non-immunogenic cell line, were inoculated into 8-week-old male and female sensory neuron intact (TRPV1wt::DTA ${ }^{\mathrm{f} / \mathrm{wt}}$ ) or ablated (TRPV1 ${ }^{\mathrm{cre}}:: \mathrm{DT} \mathrm{A}^{\mathrm{ff} / \mathrm{wt}}$ ) mice. Tumor size was measured daily using a handheld digital caliper while infiltrating $\mathrm{CD}^{+} \mathrm{T}$-cells' number and relative exhaustion were immunophenotyped by flow cytometry. Fourteen days post-tumor inoculation, we found that nociceptor-ablated mice had similar tumor volume (A, B) and weight (C) than littermate control mice. Both lines had similar levels of tumor-infiltrating CD8 ${ }^{+}$T-cells (D) as well as $\mathrm{PD}^{+}{ }^{+} \mathrm{Lag3}^{+} \mathrm{Tim}^{+}(\mathbf{E}), \mathrm{IFNy}^{+}(\mathbf{F})$, and TNFa ${ }^{+}(\mathbf{G})$ CD8 ${ }^{+}$T-cells. Data are shown as mean \pm S.E.M; two-way ANOVA (A) and unpaired Student t-test (B-G). 

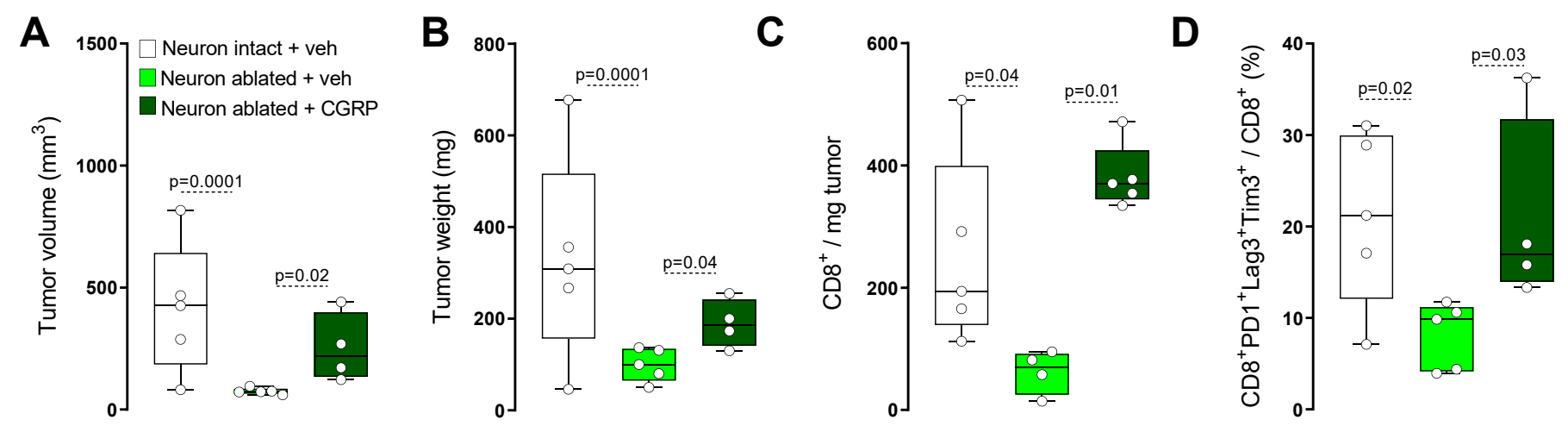

Supplementary Figure 19: Exogenous CGRP rescue tumor growth in B16F10 cells. Orthotopic B16F10-mCherrry-OVA cells $\left(100,000\right.$ cells; i.d.) were inoculated into 8 -week-old male and female sensory neuron intact (TRPV1wt::DTA ${ }^{\text {fl/wt }}$ ) or ablated (TRPV1 ${ }^{\left.\text {cre }:: D T A^{f / / w t}\right)}$ mice. Once tumors were visible $(\sim d 4)$, recombinant CGRP $(100 \mathrm{nM})$ or its vehicle were injected once daily (5 points around the tumor). The impact of CGRP injection was measured on tumor volume and weight as well as on tumorinfiltrating $\mathrm{CD}^{+} \mathrm{T}$-cells' number and exhaustion. Thirteen days post-tumor inoculation, we found that CGRP-treated nociceptor ablated mice have similar tumor growth $(\mathbf{A})$ and weight $(\mathbf{B})$ as well as infiltrating CD8 $^{+}$T-cell numbers $(\mathbf{C})$ and relative exhaustion (D) than vehicle-exposed littermate control mice. Data are shown as mean \pm S.E.M; unpaired Student $t$-test (A-D). The tumor growth curve associated is shown in figure $\mathbf{3 H}$. 

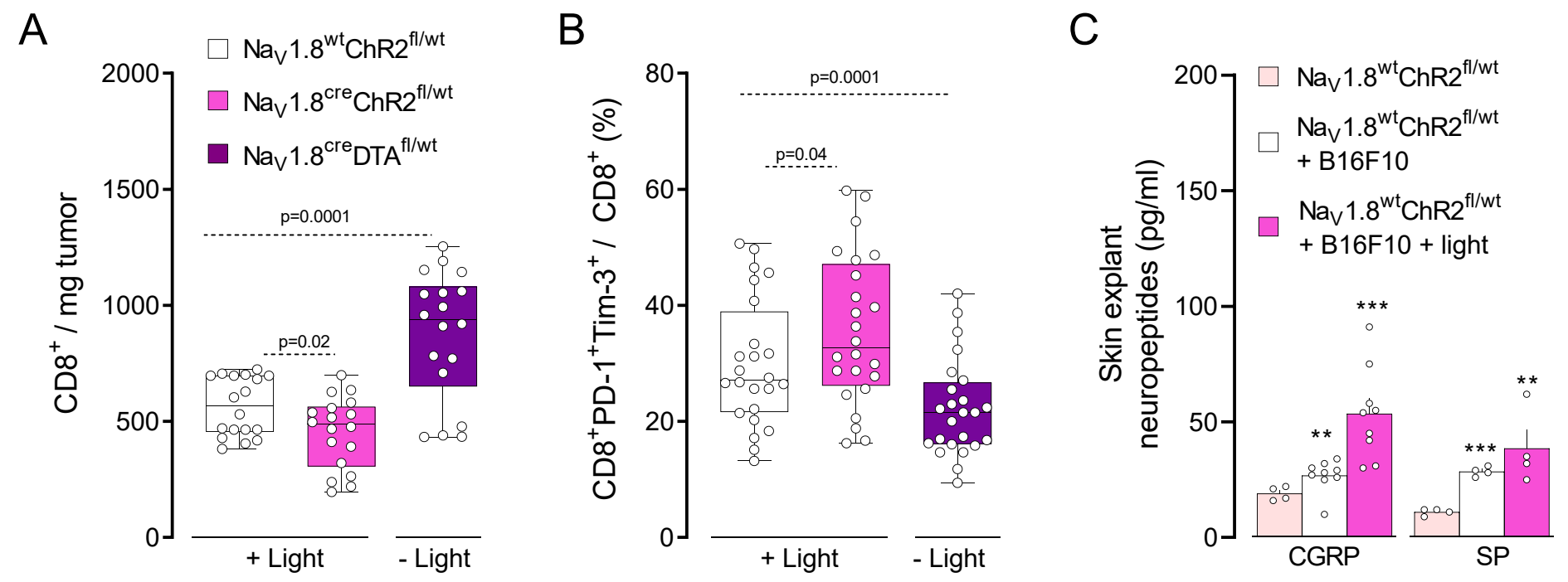

Supplementary Figure 20: Optogenetic activation of $\mathrm{Nav}_{1.8^{+}}$nociceptor neurons promotes $\mathrm{CD}^{+} \mathrm{T}$-cells exhaustion. Orthotopic B16F10 cells (200,000 cells; i.d.) were inoculated into 8-week-old male and female sensory neuron intact

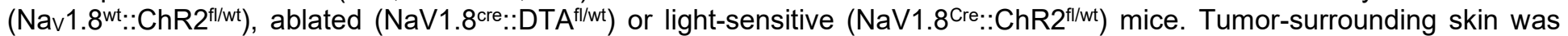
stimulated once daily by optogenetic $\left(3.5 \mathrm{~ms}, 10 \mathrm{~Hz}, 478 \mathrm{~nm}, 100 \mathrm{~mW}\right.$, giving approx. $2-6 \mathrm{~mW} / \mathrm{mm}^{2}$ with a $0.39 \mathrm{NA}$ fibre placed $5-10 \mathrm{~mm}$ from the skin for $20 \mathrm{~min}$ ). The impact of nociceptor activation or ablation was measured on the number and exhaustion of tumor-infiltrating CD8 ${ }^{+}$T-cells as well as CGRP release. Eleven days post-tumor inoculation, we found that the daily optogenetic activation of light-sensitive B16F10-inoculated skin decreased the intra-tumor number of CD8 ${ }^{+} \mathrm{T}_{\text {-cells }}(\mathbf{A})$ but increased their relative exhaustion (B; the proportion of $\left.\mathrm{PD} 1^{+} \mathrm{Tim}^{+}\right)$and the secretion of CGRP (C; assessed by ELISA in tumor-surrounding skin). Genetic ablation of Nav1.8+ lineage neurons (NaV1.8 Cre::DTA ${ }^{\mathrm{fl} / \mathrm{wt}}$ ) had the opposite effect (A-B). Mean \pm S.E.M; one-way ANOVA post hoc Bonferroni (A-C); $p$-values are shown in the figure. The tumor growth curve associated is shown in figure 31 . 

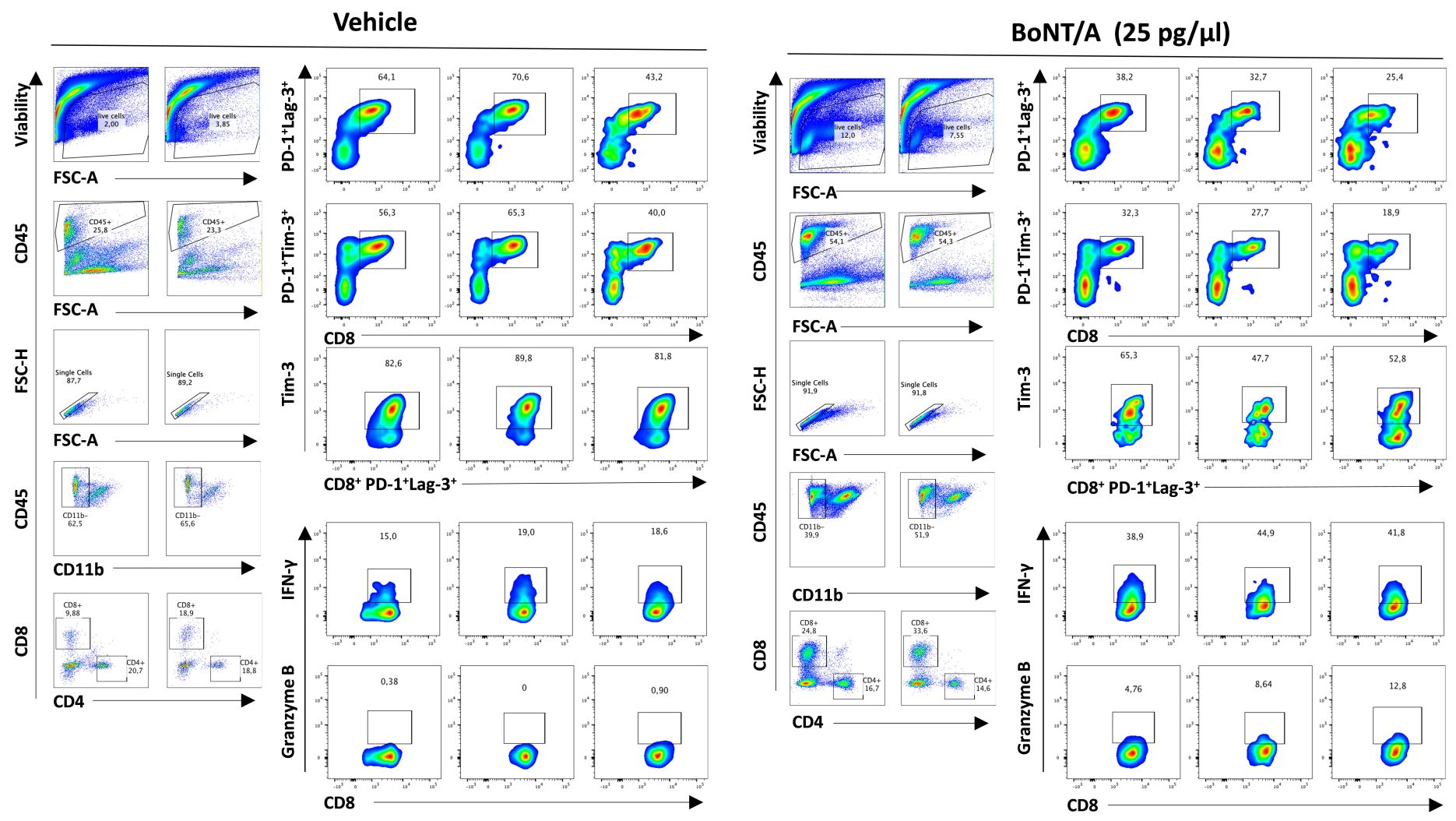

Supplementary Figure 21: Botox silencing of B16F10-innervating neurons decreases tumor growth. One and three days prior to tumor inoculation, the skin of 8-week-old male and female mice was injected with BoNT/A (25 pg/ $/$ l; i.d.) or its vehicle. One day following the last injection, orthotopic B16F10 cells $(500,000$ cells; i.d.) were inoculated into the area pre-exposed to BoNT/A. The impact of nociceptor neuron silencing on tumor-infiltrating CD8 ${ }^{+}$T-cell exhaustion was immunophenotyped by flow cytometry fourteen days post-tumor inoculation. The representative gating strategy used in figure $4 B$ is shown ( $n=3$ /groups). 
A

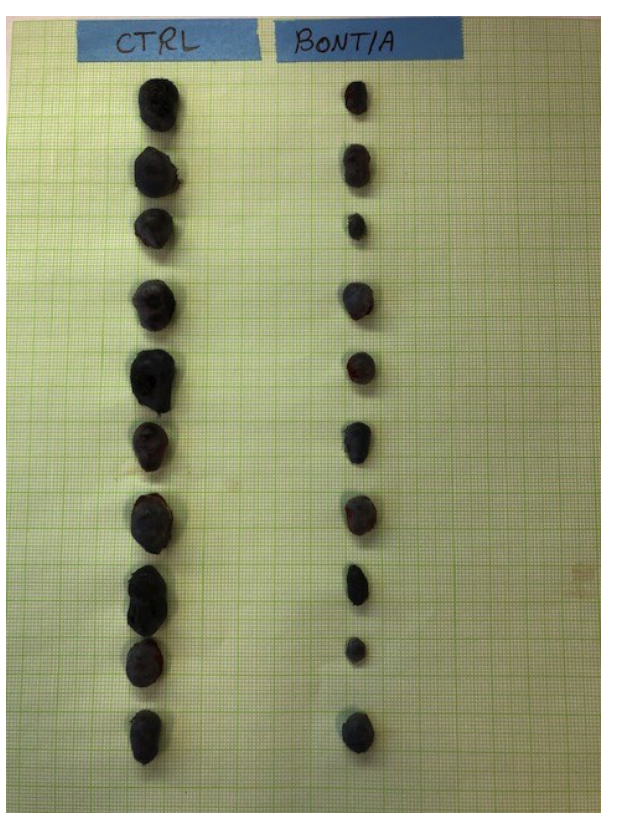

B

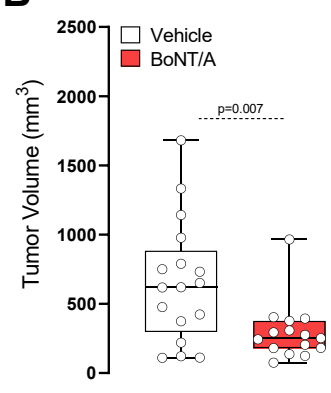

E

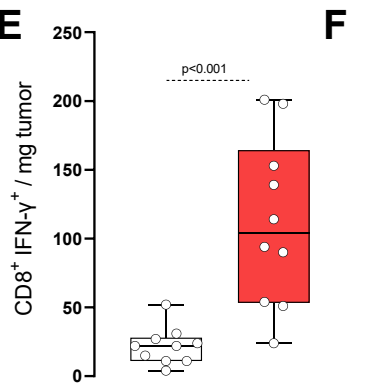

C

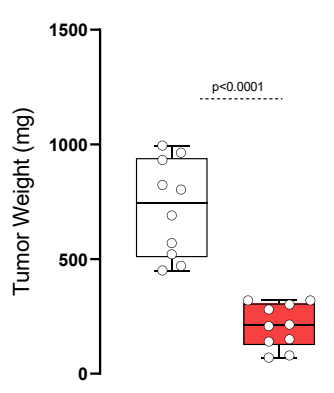

D
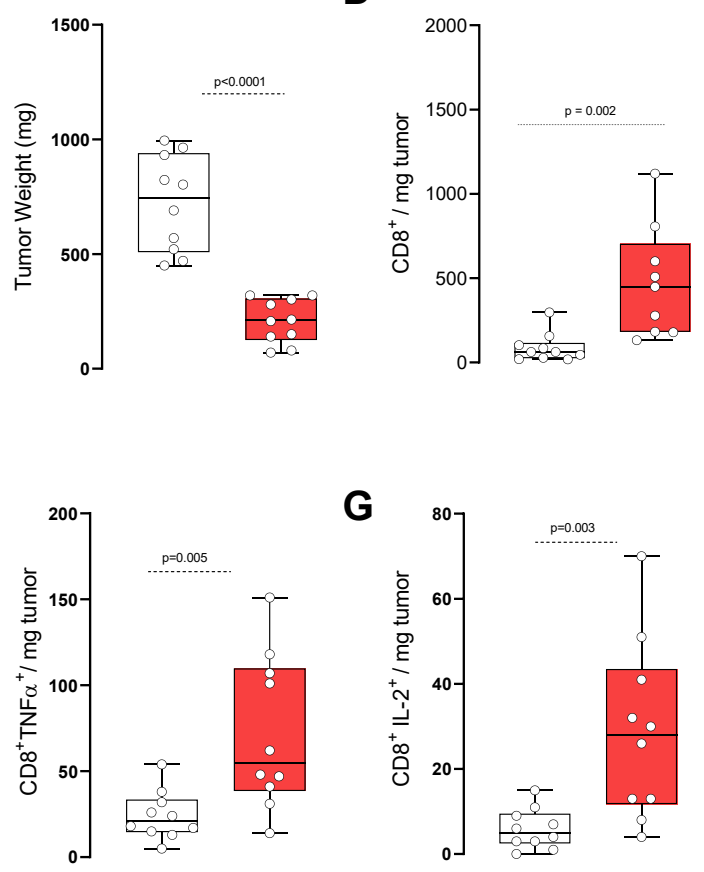

G

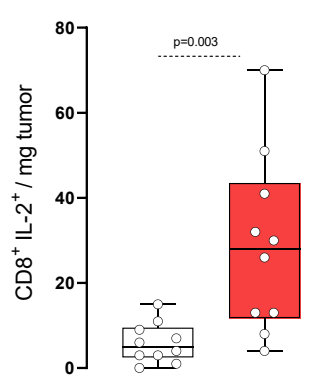

Supplementary Figure 22: Botox silencing of B16F10-innervating neurons decreases tumor growth. One and three days prior to tumor inoculation, the skin of 8-week-old male and female mice was injected with BoNT/A (25 pg/ul; i.d.) or its vehicle. One day following the last injection, orthotopic B16F10 cells $(500,000$ cells; i.d.) were inoculated into the area pre-exposed to BoNT/A. The impact of neuron silencing on tumor size and tumor-infiltrating CD8 ${ }^{+}$T-cell exhaustion were measured. Fourteen days post-tumor inoculation, we found that the tumor volume $(\mathbf{A}, \mathbf{B})$ and weight $(\mathbf{C})$ were reduced in mice treated with BoNT/A. In parallel, we found that silencing tumor-innervating neurons increased the intra-tumoral number of total (D), IFNy ${ }^{+}(\mathbf{E}), \mathrm{TNFa}^{+}$ $(\mathbf{F})$, and IL-2+ $(\mathbf{G}) \mathrm{CD}^{+}$T-cells. Data are shown as mean \pm S.E.M; unpaired Student $t$-test $(\mathbf{A}-\mathbf{G}) ; p$-values are shown in the figure. The tumor growth curve associated is shown in figure $\mathbf{4 A}$. 


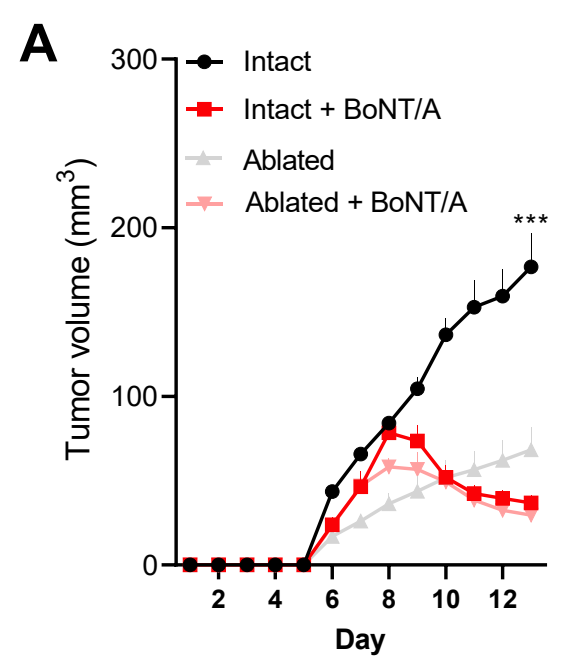

B

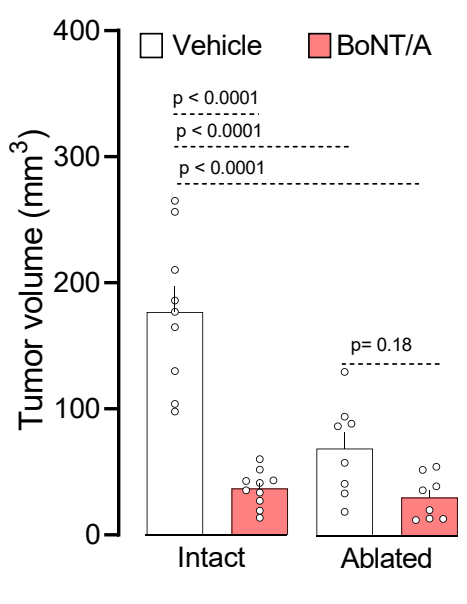

C

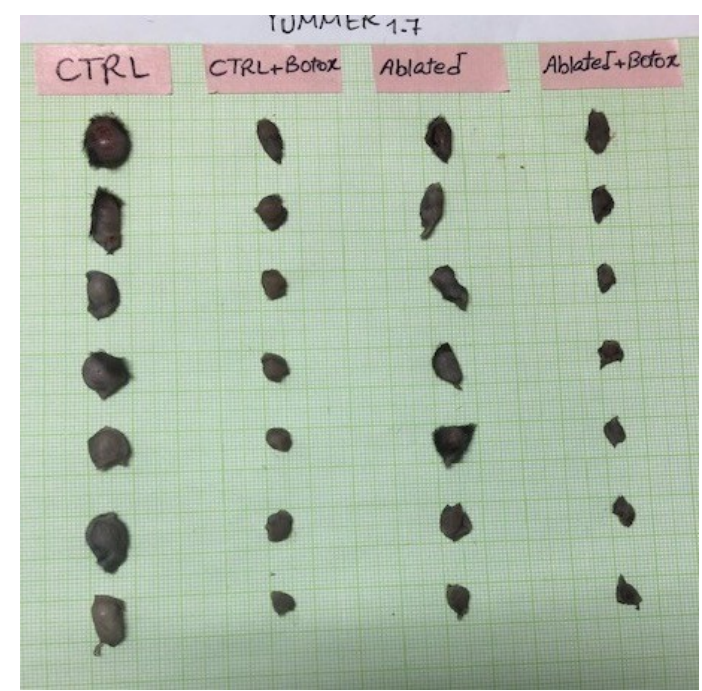

Supplementary Figure 23: Botox silencing of YUMMER1.7-innervating neurons decreases tumor growth. One and three days prior to tumor inoculation, the skin of 8-week-old male and female sensory neuron intact or ablated mice was injected with BoNT/A (25 pg/ul; i.d.) or its vehicle. One day following the last injection, orthotopic YUMMER1.7 cells $(500,000$ cells; i.d.) were inoculated into the area pre-exposed to BoNT/A. The effects of nociceptor neuron ablation on tumor size and volume were measured. Thirteen days post-tumor inoculation, we found that the tumor growth $(\mathbf{A})$, volume $(\mathbf{B})$, and size $(\mathbf{C})$ were lower in mice treated with BoNT/A or in sensory neuron-ablated mice. BoNT/A had no additive effects when administered to sensory neuron-ablated mice (A-C). Data are shown as mean \pm S.E.M; two-way (A) or one-way (B) ANOVA post hoc Bonferroni; $p$ values are shown in the figure. 
Vehicle

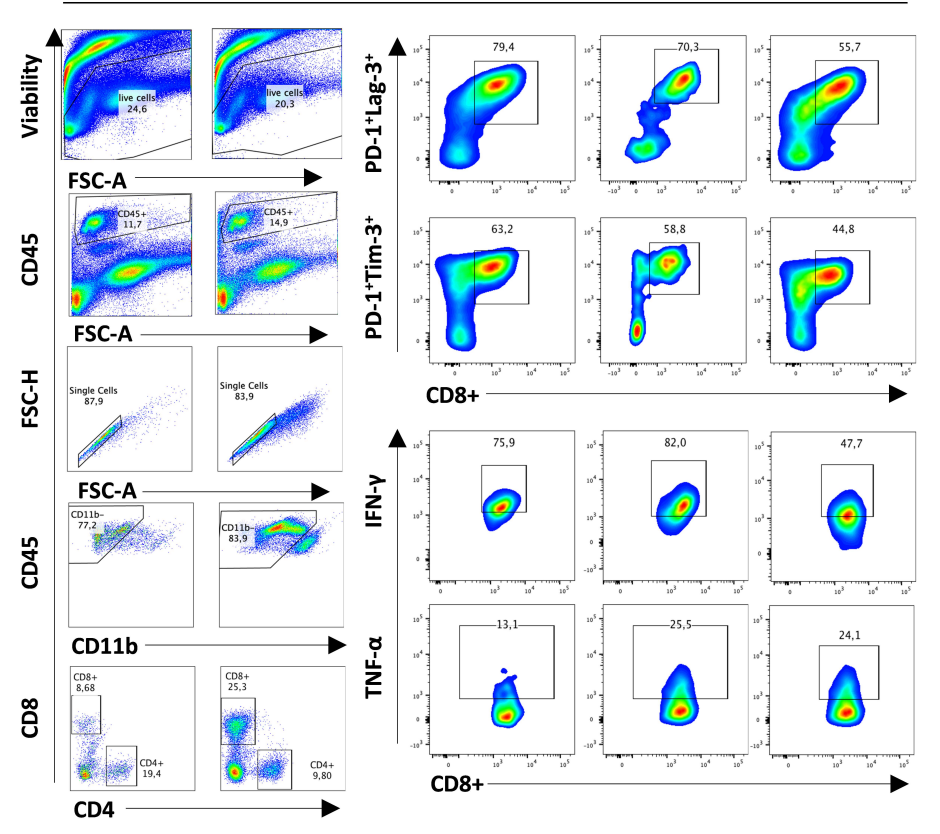

QX-314 (0.3\%)

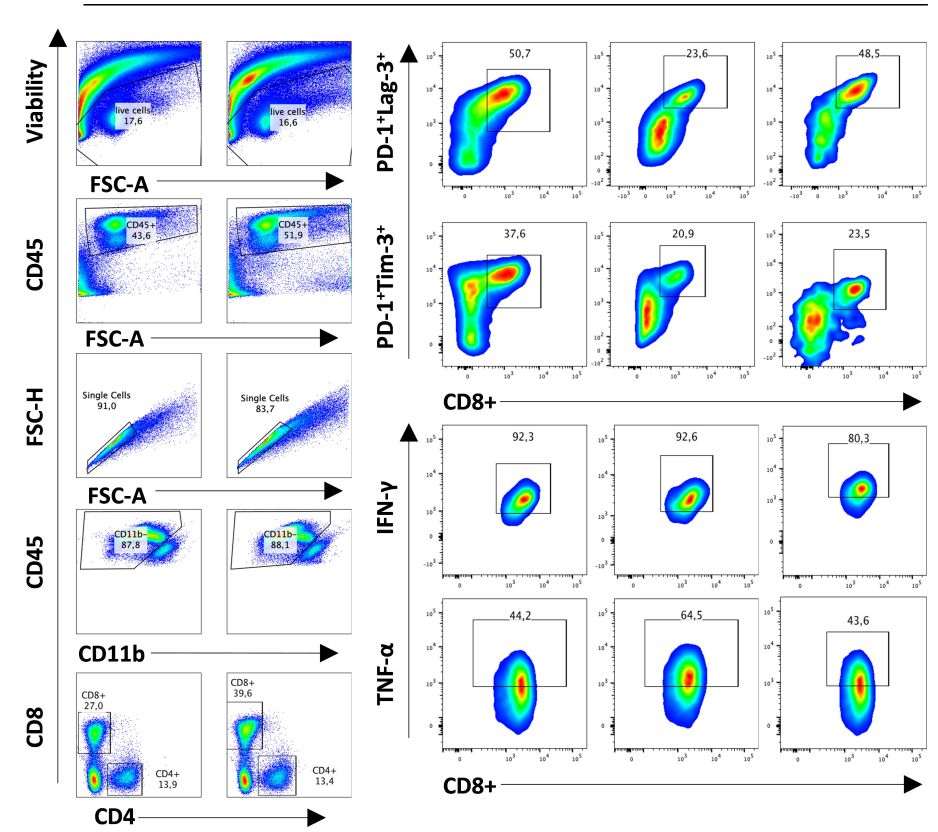

Supplementary Figure 24: QX-314 silencing of B16F10-innervating neurons decreases tumor growth. Orthotopic B16F10-mCherry-OVA cells (500,000 cells; i.d.) were inoculated into 8-week-old male and female mice. Starting one day postinoculation, QX-314 (0.3\%; i.d.; 5 sites) was injected once daily around the tumor. The impact of nociceptor neuron silencing on tumor-infiltrating CD8 ${ }^{+}$T-cell exhaustion was immunophenotyped by flow cytometry sixteen days post-tumor inoculation. The representative gating strategy used in figure $\mathbf{4 D}$ is shown ( $n=3 /$ groups). 
A

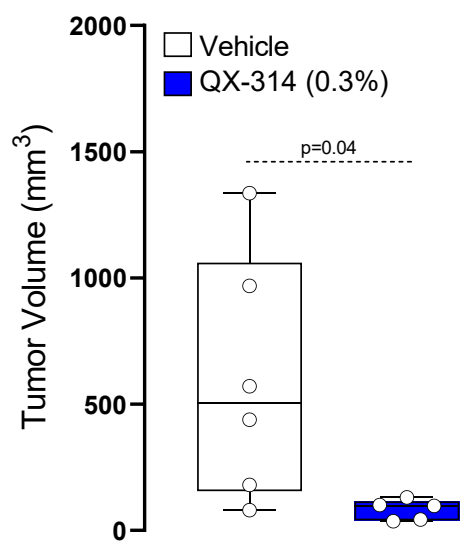

D

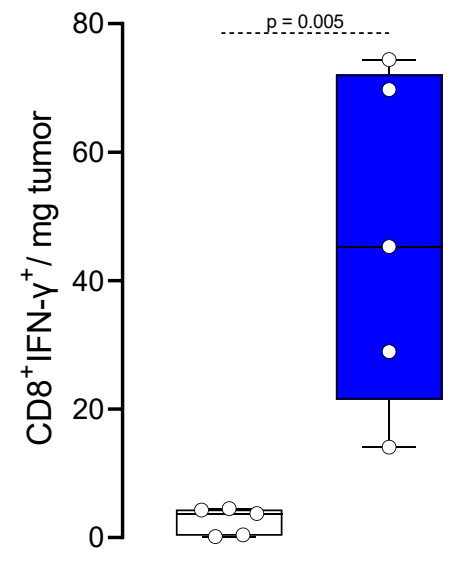

B

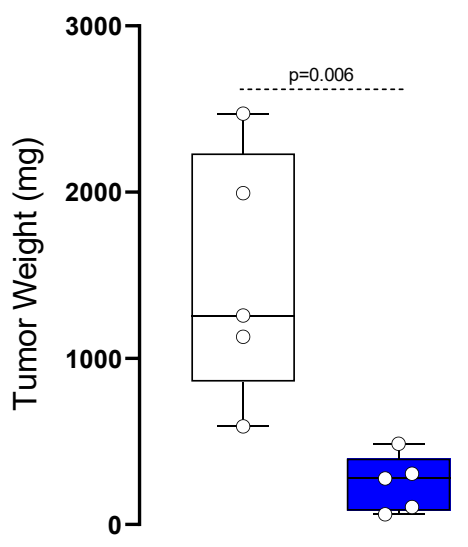

$\mathbf{E}$

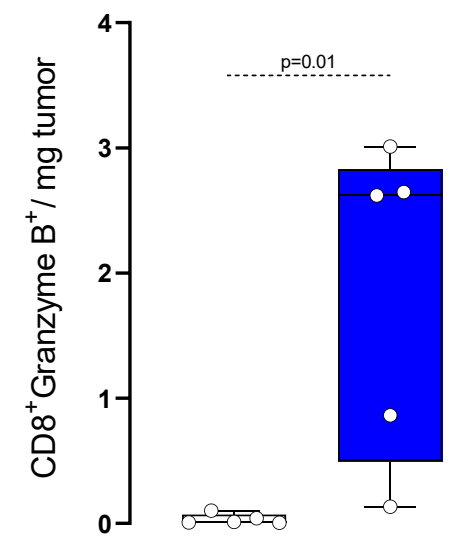

C

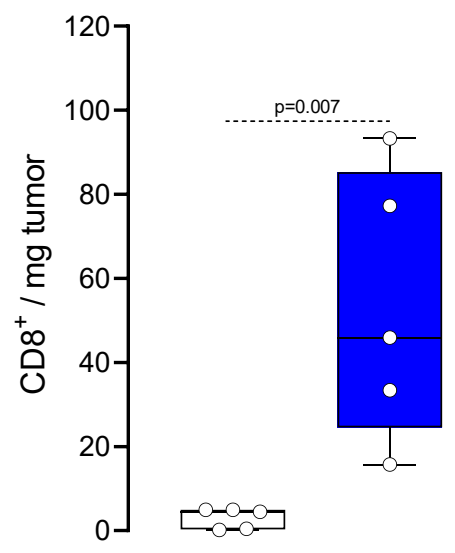

$\mathbf{F}$

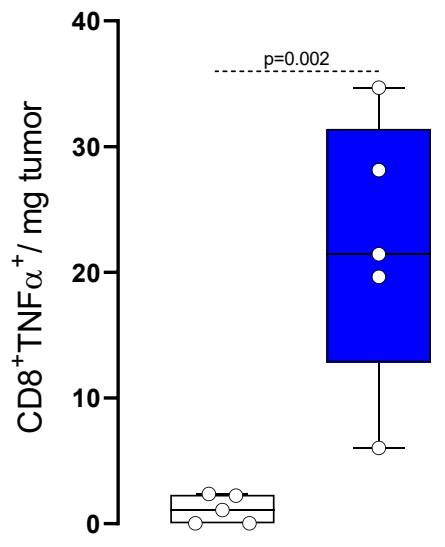

Supplementary Figure 25: QX-314 silencing of B16F10-innervating neurons decreases tumor growth. Orthotopic B16F10-mCherry-OVA cells (500,000 cells; i.d.) were inoculated into 8-week-old male and female mice. Starting one day postinoculation, QX-314 (0.3\%; i.d.; 5 sites) was injected once daily around the tumor. The impact of nociceptor neuron silencing on tumor size and tumor-infiltrating $\mathrm{CD} 8^{+} \mathrm{T}$-cell exhaustion were measured. Sixteen days post-tumor inoculation, we found that the tumor volume (A) and weight (B) were lower in mice treated with QX-314. Silencing tumor-innervating neurons also increased the intra-tumoral number of total (C), IFNy+ (D), Granzyme $\mathrm{B}^{+}(\mathbf{E})$, and $\mathrm{TNFa}^{+}(\mathbf{F}) \mathrm{CD}^{+} \mathrm{T}^{+}$-cells. Data are shown as mean \pm S.E.M; unpaired Student $t$-test $(\boldsymbol{A}-\mathbf{G}) ; p$-values are shown in the figure. The tumor growth curve associated is shown in figure $4 C$. 
Vehicle

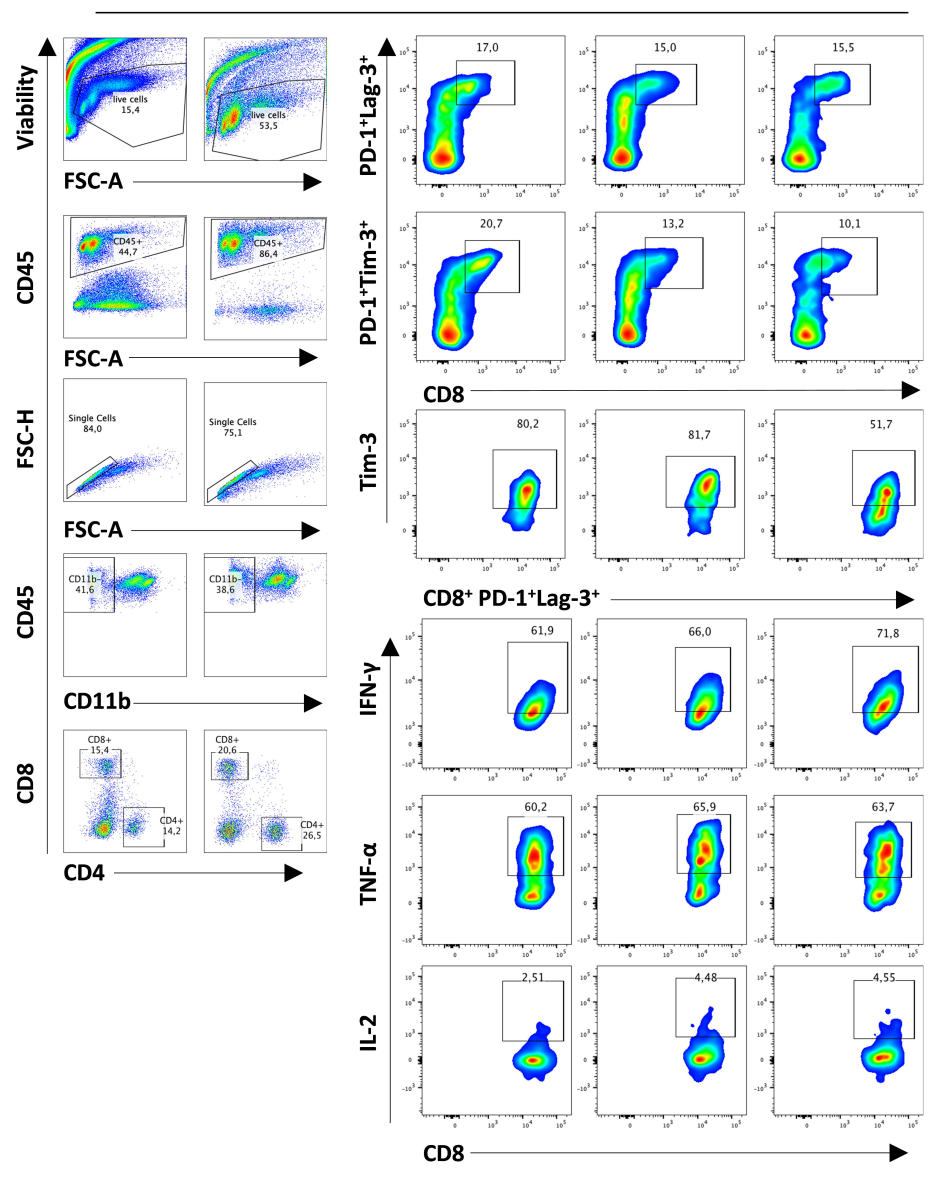

BIBN4096 (5mg/kg)

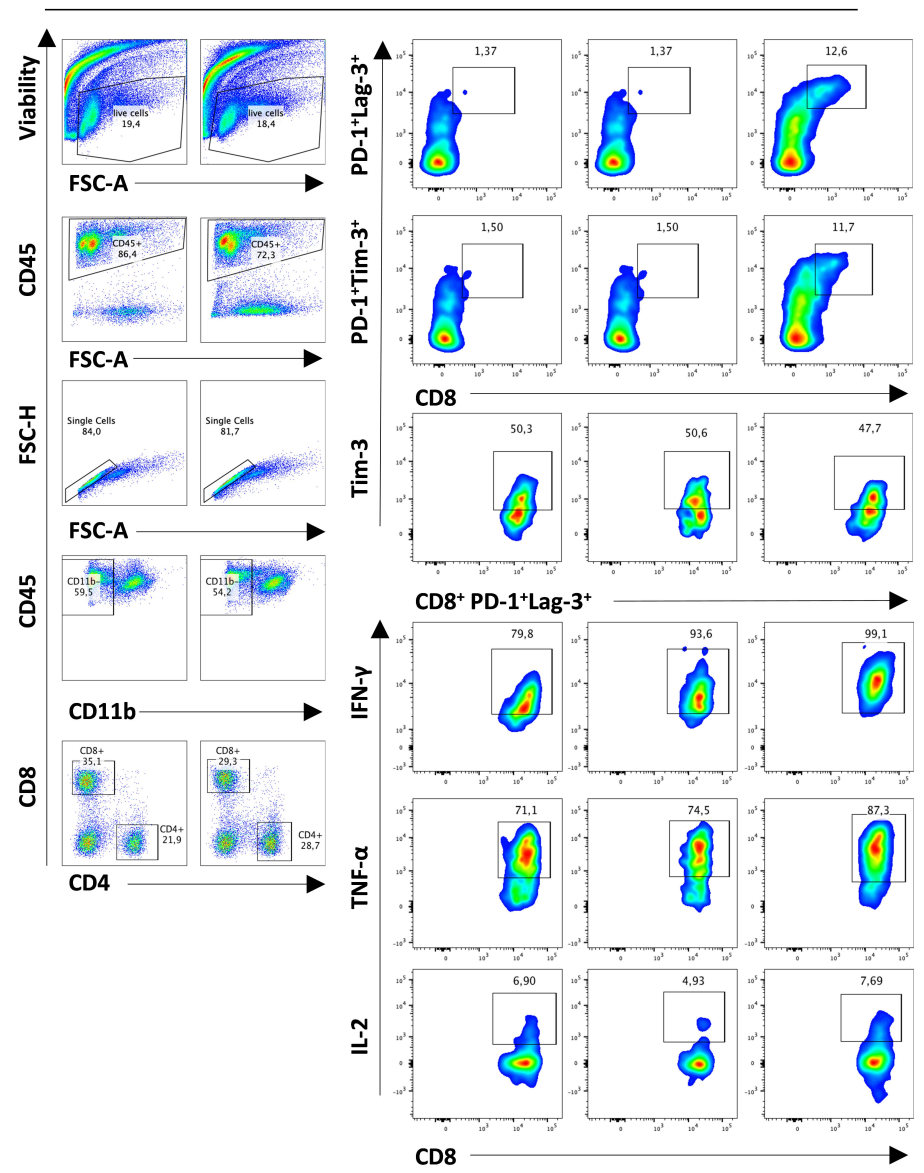

Supplementary Figure 26: RAMP1 antagonism prevents CD8 ${ }^{+}$T-cell exhaustion. Orthotopic B16F10-mCherry-OVA cells (500,000 cells; i.d.) were inoculated into 8-week-old male and female mice. BIBN4096 $(5 \mathrm{mg} / \mathrm{kg})$ or its vehicle was administered (i.p.) on days $6,8,10,12$, and 14. The impact of RAMP1 antagonism on tumor-infiltrating CD8 ${ }^{+}$T-cell exhaustion was immunophenotyped by flow cytometry sixteen days post-tumor inoculation. The representative gating strategy used in figure $4 \mathrm{H}$ is shown ( $n=3 /$ groups). 
A

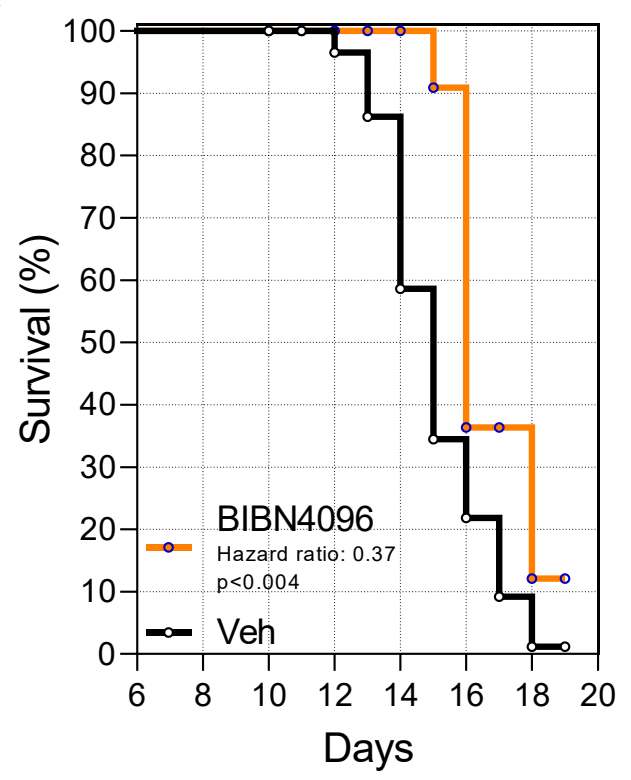

B

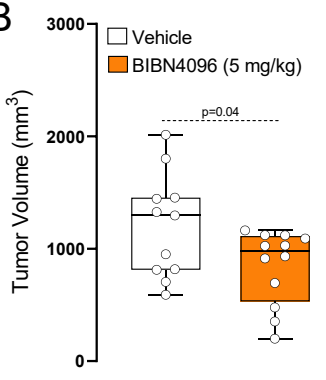

$\mathrm{E}$

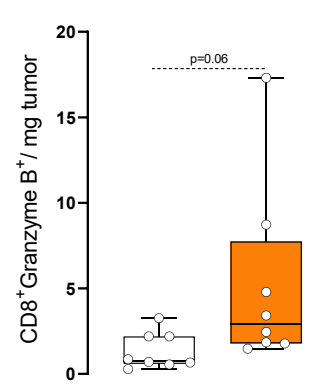

C
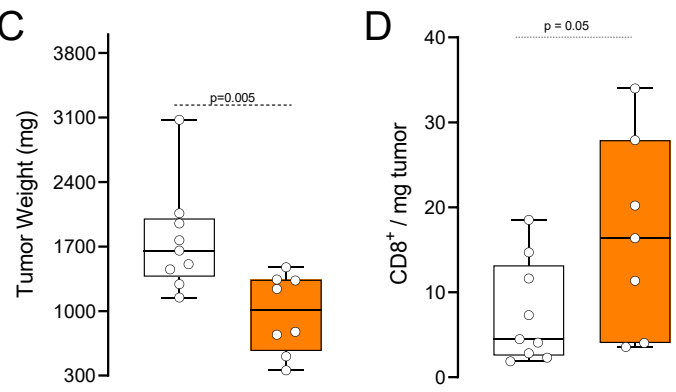

$\mathrm{F}$

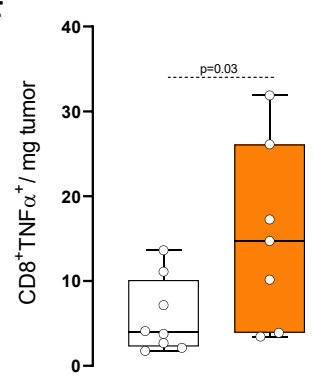

Supplementary Figure 27: RAMP1 antagonism prevents CD8 ${ }^{+}$T-cell exhaustion. Orthotopic B16F10-mCherry-OVA cells $(500,000$ cells; i.d.) were inoculated into 8-week-old male and female mice. BIBN4096 $(5 \mathrm{mg} / \mathrm{kg})$ or its vehicle was administered (i.p.) every second day. The impact of RAMP1 antagonism on survival, tumor size and tumor-infiltrating CD8 ${ }^{+} \mathrm{T}$-cell exhaustion were measured. Compared to vehicle-exposed mice, B16F10-bearing mice succumbed at a 2.6-fold higher rate $(p \leq 0.04)$ than BIBN4096-exposed mice (assessed on day 19 and determined by reaching a volume of $\sim 800 \mathrm{~mm}^{3}$ or other ethical endpoints). In other groups of mice, we found that sixteen days post-B16F10 inoculation that the tumor volume (B) and weight (C) were reduced BIBN4096-treated mice. Blocking RAMP1 also increased the intra-tumoral number of total (D), Granzyme $B^{+}(E)$, and $\mathrm{TNFa}^{+}(\mathbf{F}) \mathrm{CD}^{+}$T-cells. Data are shown as mean \pm S.E.M; Mantel-Cox regression (A) and unpaired Student $t$-test $(\boldsymbol{B}-\boldsymbol{F}) ; p$ values and $n$ are shown in the figure. The tumor growth curve associated is shown in figure $\mathbf{4 G .}$ 


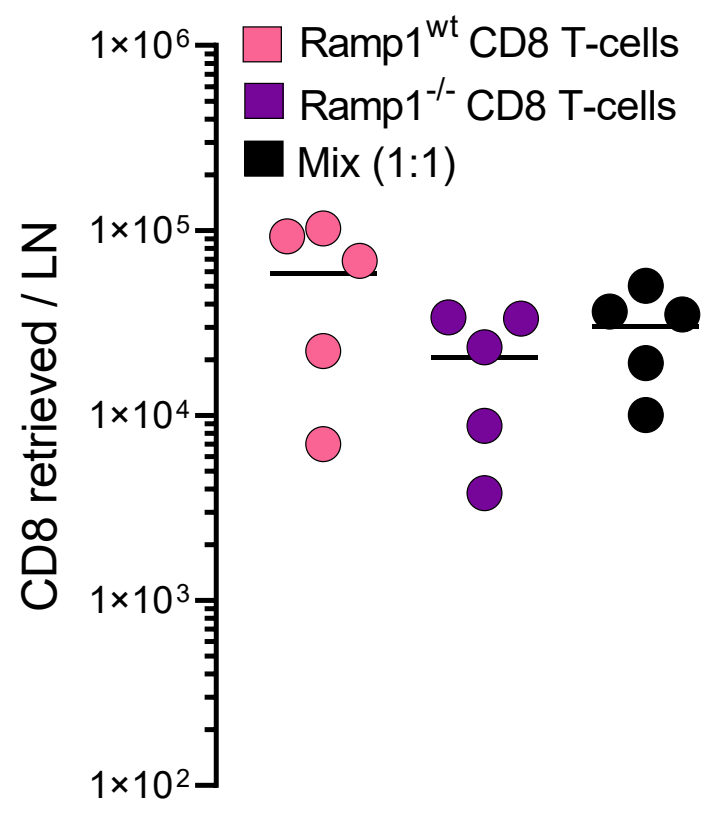

Supplementary Figure 28: Rag1-/- mice were successfully transplanted with CD8 ${ }^{+}$T-cells. Naive splenocytes CD8 ${ }^{+}$Tcells were FACS-purified from wildtype $\left(\mathrm{CD} 45.1^{+}\right)$or RAMP1-- $\left(\mathrm{CD} 45.2^{+}\right)$mice, cultured and amplified. 8-week-old female Rag11- mice were transplanted (i.v.; $2.5 \times 10^{6}$ cells) with RAMP1-/- or RAMP1wt $C D 8^{+}$T-cells or 1:1 mix of RAMP1-- and RAMP1wt $C D 8^{+}$ T-cells. One-week post-transplantation, the mice were inoculated with B16F10-mCherry-OVA cells $(500,000$ cells; i.d.). Ten days post-tumor inoculation, we retrieved a similar number of tumor-draining lymph node CD8 ${ }^{+} \mathrm{T}$-cells across the three tested groups. Data are shown as mean \pm S.E.M; one-way ANOVA post hoc Bonferroni. 

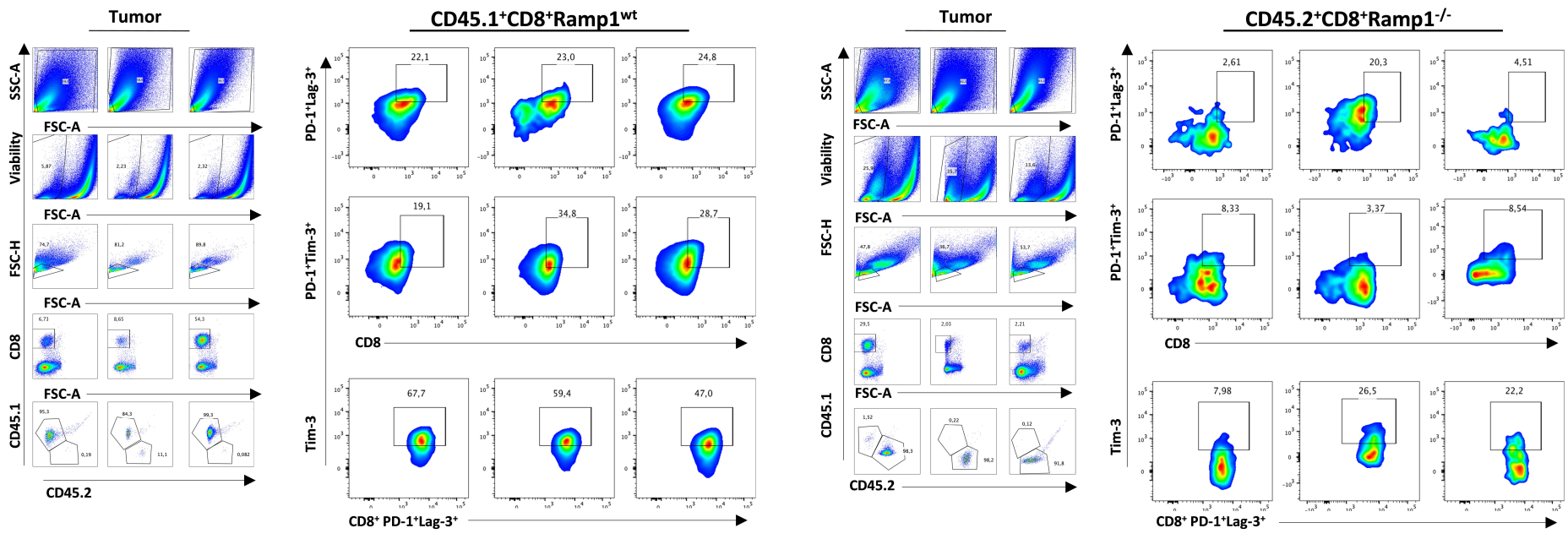

Supplementary Figure 29: RAMP1-/- $\mathrm{CD}^{+} \mathrm{T}$-cells are resistant to immune exhaustion. Naive splenocytes $\mathrm{CD} 8^{+} \mathrm{T}_{\text {-cells }}$ were FACS-purified from wildtype $\left(\mathrm{CD} 45.1^{+}\right)$or RAMP1-/- $\left(\mathrm{CD} 45.2^{+}\right)$mice, cultured and amplified. 8-week-old female Rag1 $1^{-/-}$ mice were transplanted (i.v.; $2.5 \times 10^{6}$ cells) RAMP1-- or RAMP1wt $\mathrm{CD} 8^{+}$T-cells. One-week post-transplantation, the mice were inoculated with B16F10-mCherry-OVA cells (500,000 cells; i.d.) and the CD8 ${ }^{+}$T-cell exhaustion was immunophenotyped by flow cytometry ten days post tumor inoculation. The representative gating strategy used in figure $4 \mathrm{~K}$ is shown (n=3/groups). 

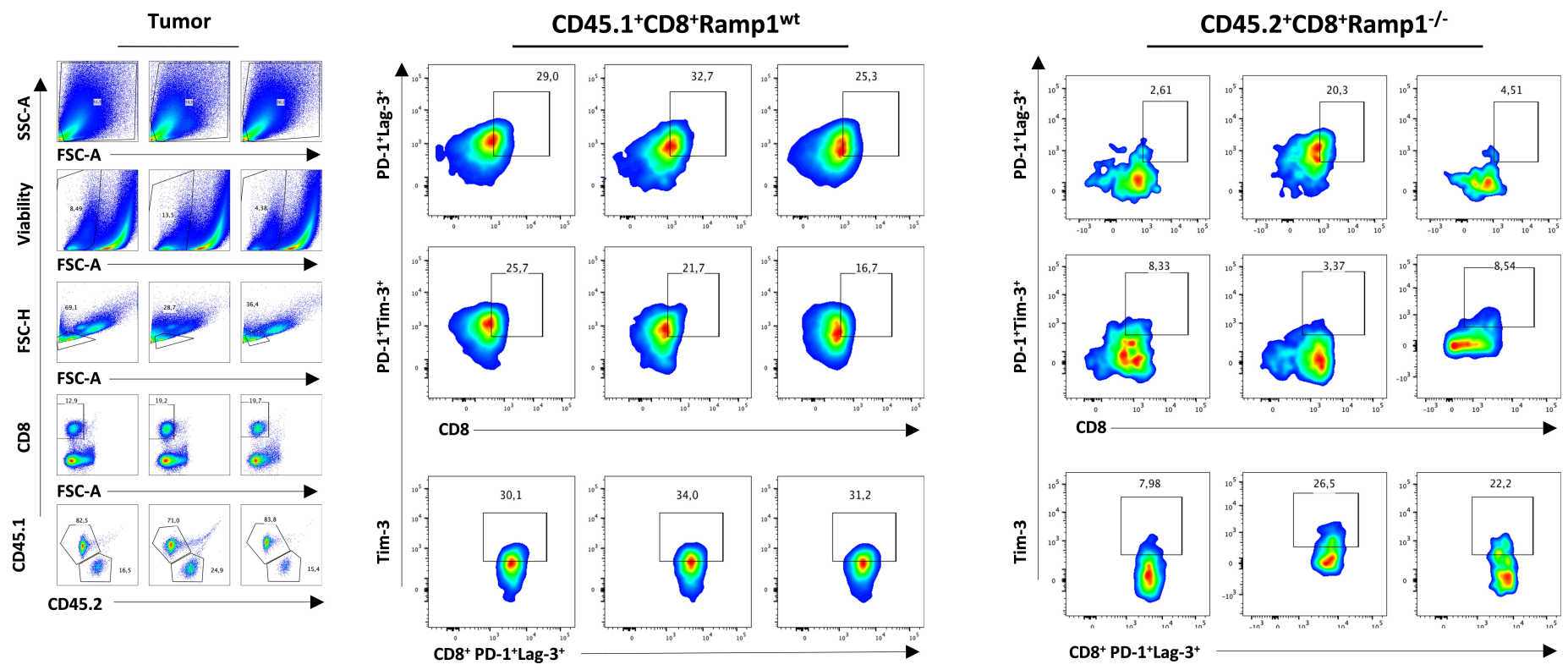

Supplementary Figure 30: RAMP1-l- $\mathrm{CD}^{+} \mathrm{T}$-cells are resistant to immune exhaustion. Naive splenocytes $\mathrm{CD} 8^{+} \mathrm{T}_{\text {-cells }}$ were FACS-purified from wildtype $\left(\mathrm{CD} 45.1^{+}\right)$or RAMP1-/ $\left(\mathrm{CD} 45.2^{+}\right)$mice, cultured and amplified. 8-week-old female Rag1 $1^{-/-}$ mice were transplanted (i.v.; $2.5 \times 10^{6}$ cells) with a 1:1 mix of RAMP1-- and RAMP1wt CD8 ${ }^{+}$T-cells. One-week posttransplantation, the mice were inoculated with B16F10-mCherry-OVA cells $\left(500,000\right.$ cells; i.d.) and the CD8 ${ }^{+}$T-cell exhaustion was immunophenotyped by flow cytometry ten days post tumor inoculation. The representative gating strategy used in figure $4 L$ is shown ( $n=3 /$ groups). 


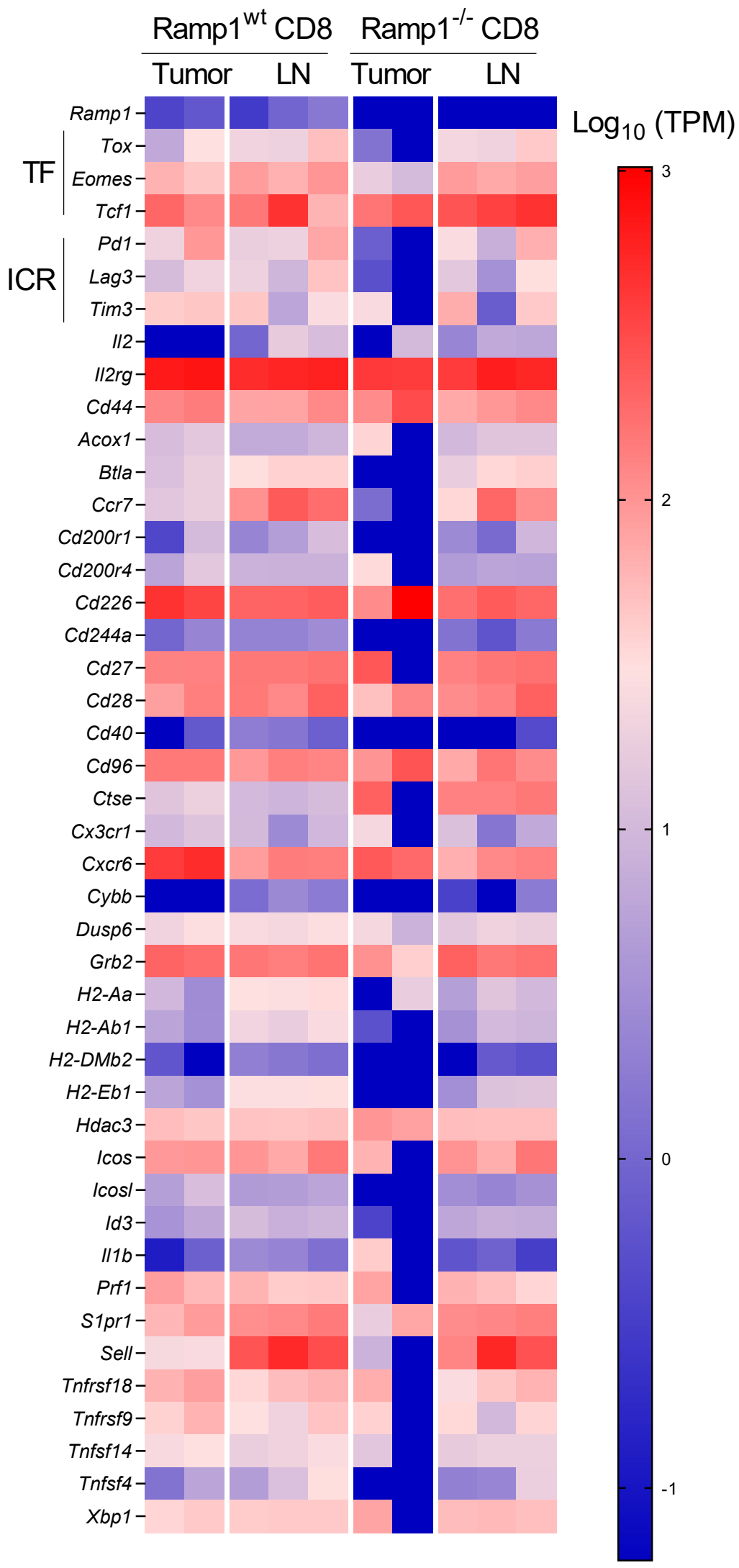

Supplementary Figure 31: RNA sequencing confirmed that RAMP1 ${ }^{--} \mathrm{CD}^{+}{ }^{+}$T-cells are exhausted. Naive splenocytes $\mathrm{CD}^{+}{ }^{+} \mathrm{T}$-cells were FACS-purified from wild-type (CD45.1 $\left.1^{+}\right)$or RAMP1-/$\left(\mathrm{CD} 45.2^{+}\right)$mice, cultured and amplified. 8-week-old female Rag1-/- mice were transplanted (i.v.) with a 1:1 mix of 5,000,000 RAMP1-/ and RAMP1wt $\mathrm{CD}^{+}{ }^{+} \mathrm{T}-$ cells. One-week post-transplantation, the mice were inoculated with B16F10-mCherry-OVA cells $(500,000$ cells; i.d.). On day 14 , tumor and draining lymph node were harvested, and RAMP1- $\left(\mathrm{CD} 45.1^{+}\right)$and RAMP1wt $\left(C D 45.1^{+}\right) \mathrm{CD}^{+}$T-cells were FACSpurified and RNA sequenced. When compared with RAMP1wt, RAMP1-- CD8 ${ }^{+}$T-cells showed reduced expression of the exhaustion marker Ctla4, Havcr2 (Tim3), Lag3, and Pd1. In tumor-draining lymph nodes, the expression of exhaustion markers was similar across the two tested groups. Data are shown as heatmaps as $\log _{10}$ of transcript per millions. 


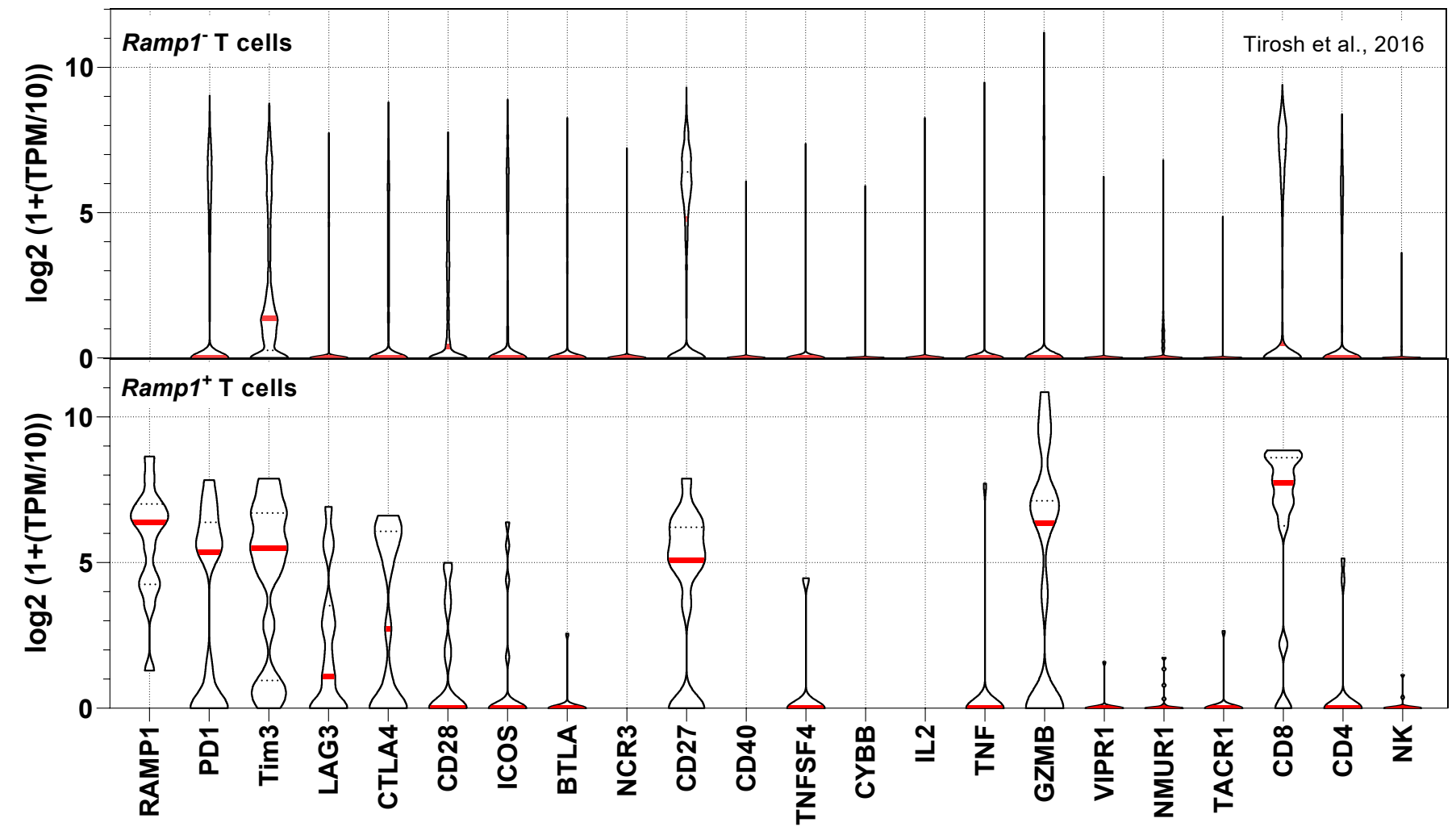

Supplementary Figure 32: Single-cell RNA sequencing of human melanoma. In-silico analysis of single-cell RNA sequencing of human melanoma-infiltrating T-cells revealed that RAMP1 ${ }^{+} \mathrm{T}$-cells downregulated IL-2 expression and strongly overexpressed several immune checkpoint receptors (PD1, Tim3, Lag3, CTLA-4, CD28, ICOS, BTLA, CD27) in comparison to RAMP1- T-cells. Individual cell data are shown as a $\log _{2}$ of $1+$ (transcript per million / 10). Experimental details and cell clustering were defined in Tirosh et al. (46). 


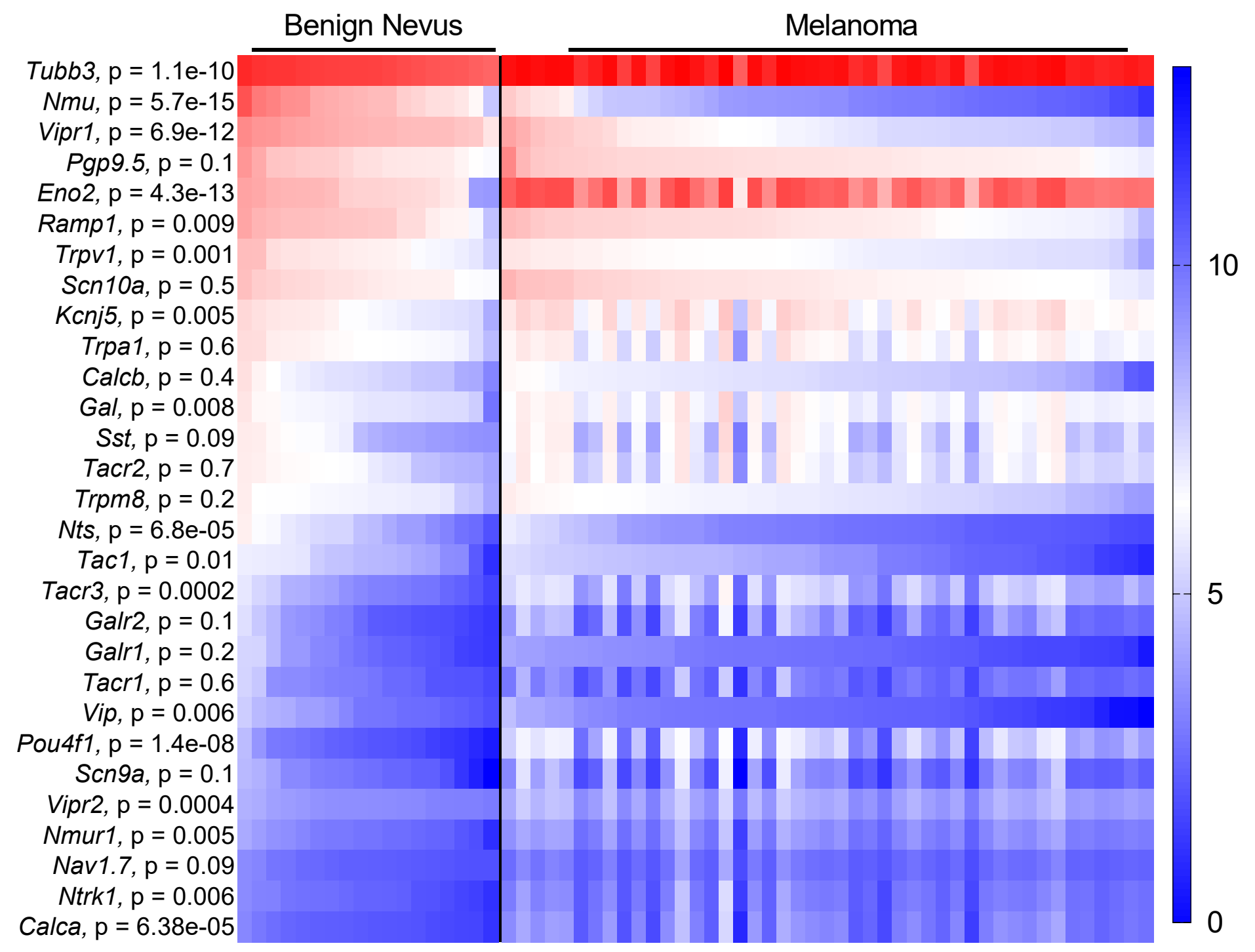

Supplementary Figure 33: Increased transcript expression of neuronal-associated genes was found in melanoma biopsies. Forty-five cutaneous melanomas and 18 benign melanocytic skin nevus biopsies transcriptome were profiled using Affymetrix U133A microarrays ${ }^{46}$. In-silico analysis of this dataset revealed that cutaneous melanoma heightened expression levels of Calca, Ramp1, Pouf4f1, Eno2, and Tubb3, as well as other neuronal-enriched genes. Heatmaps data are shown as $\log _{2}$ (median centred intensity); unpaired Student t-test; $p$-value shown in the figure. Experimental details were defined in Haqq et al. ${ }^{46}$. 

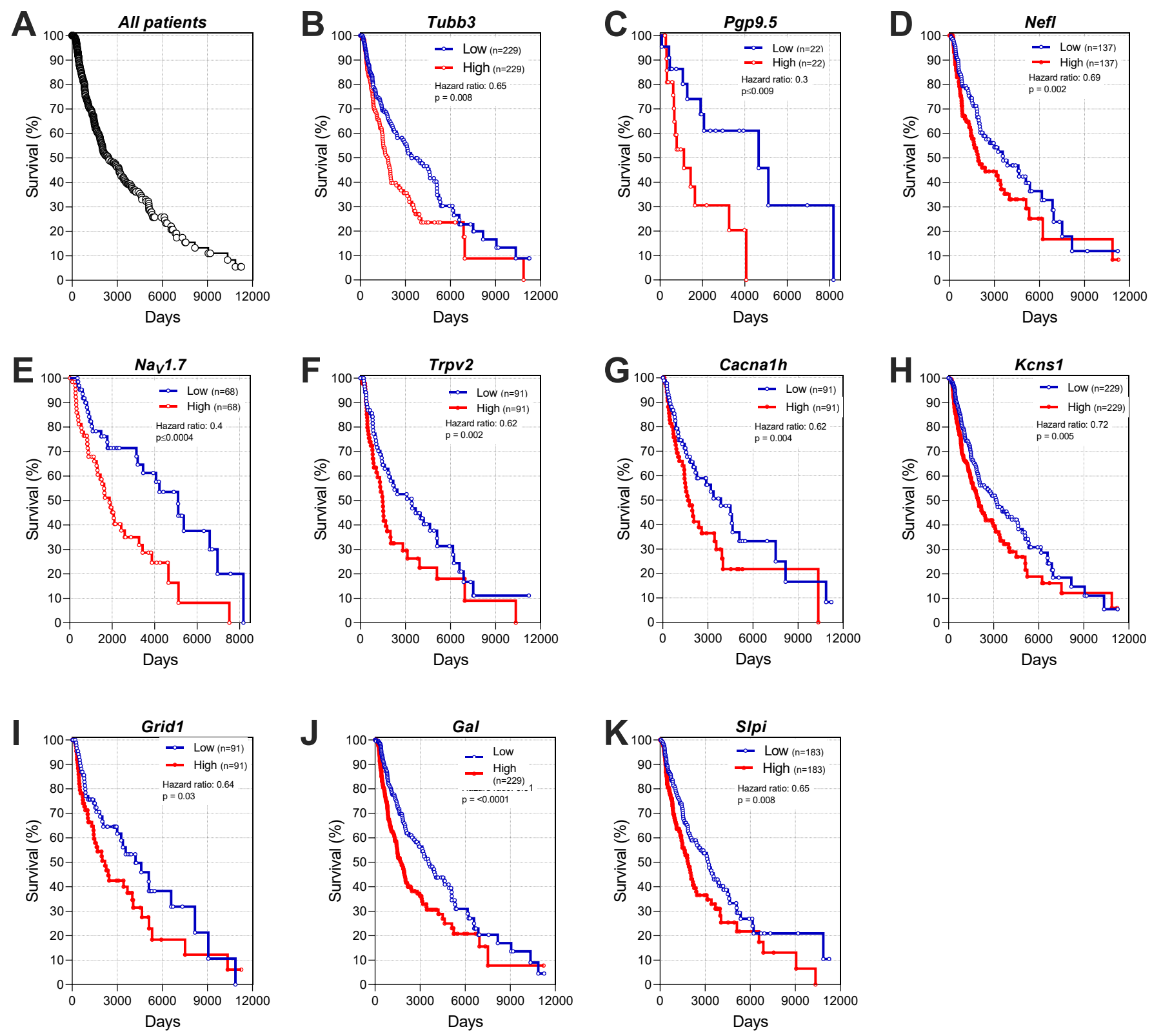

Supplementary Figure 34: Increased neuronal-associated gene transcript expression in melanoma patients' biopsies correlate with worsened survival. In-silico analysis of TCGA ${ }^{62}$ data link the survival rate of 459 melanoma patients with their relative expression levels of various genes of interest (determined by bulk RNA sequencing). Kaplan-Meier curves show the patients' survival after segregation in two groups defined by their low or high expression of a gene of interest. Increased gene expression (labelled as high; red curve) of Tubb3 (B), Pgp9.5 (C), Nav1.7 (E), and Slpi (K) in biopsy correlate with decreased patient survival $(\mathrm{p} \leq 0.05)$. Mantel-Haenszel Hazard ratio and the number of patients included in each analysis are shown in the figure. Experimental details were defined in Cancer Genome Atlas ${ }^{62}$. 


\section{Patient's intratumoral $\mathrm{CD}^{+}{ }^{+}$T-cells expression profile}
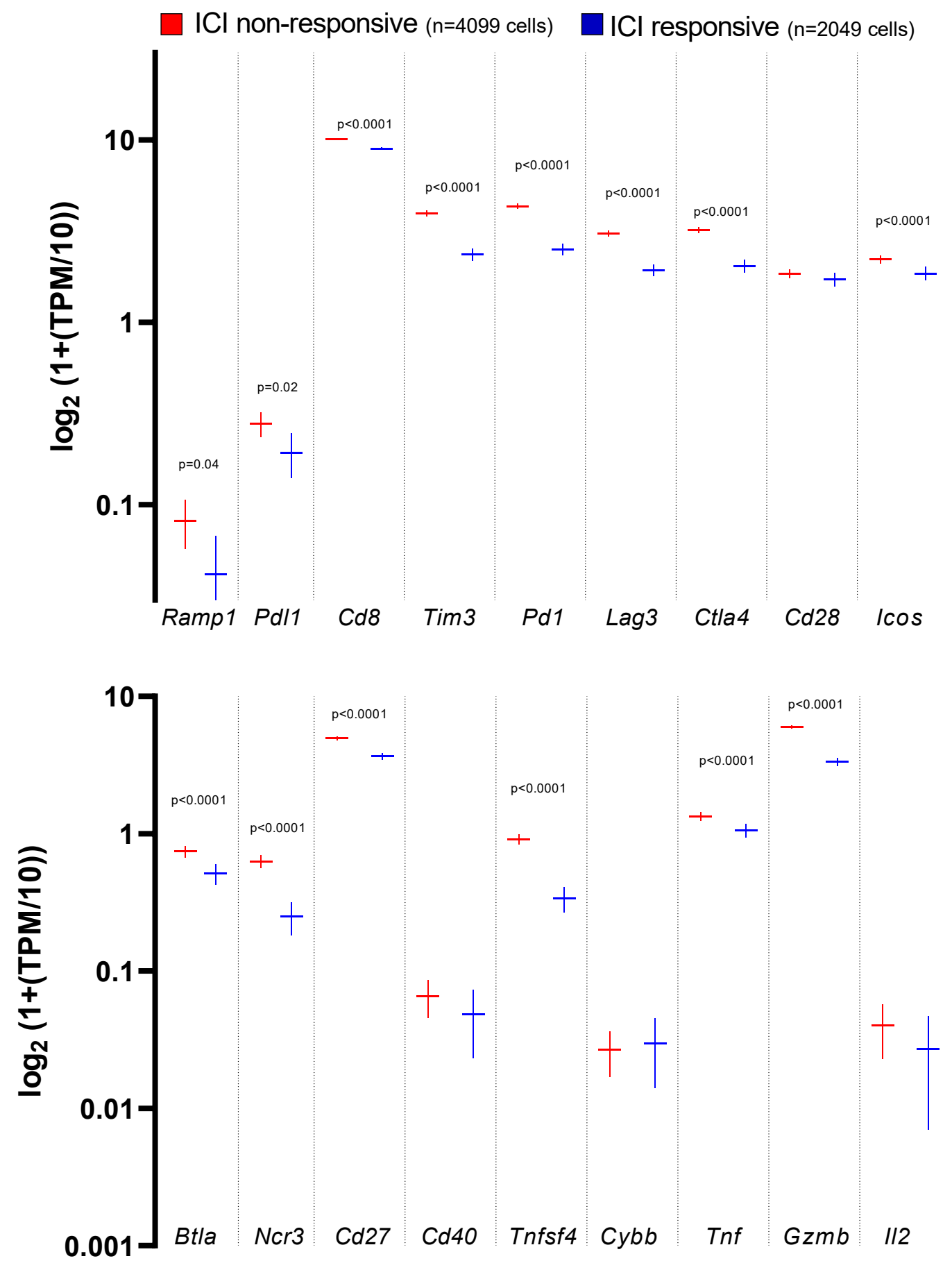

Supplementary Figure 35: RAMP1 expression in patients' melanoma infiltrating CD8 $^{+} \mathrm{T}^{-}$-cells correlates with poor responsiveness to ICI. Based on their clinical response to immune checkpoint blocker, Jerby-Arnon et al. clustered melanoma patients into two groups defined as immune checkpoint receptor responsive or resistant ${ }^{44}$. In-silico analysis of single-cell RNA sequencing of patients' biopsies revealed that tumor-infiltrating CD8 ${ }^{+}$T-cells from immune checkpoint receptor-resistant patients significantly overexpressed Ramp1 (2.0-fold), Pd1 (1.7-fold), Lag3 (1.6-fold), Ctla4 (1.6-fold), and Tim3 (1.7-fold). Individual cell data are shown as a $\log _{2}$ of $1+($ transcript per million / 10). Experimental details and cell clustering were defined in Jerby-Arnon et al. ${ }^{44}$. 

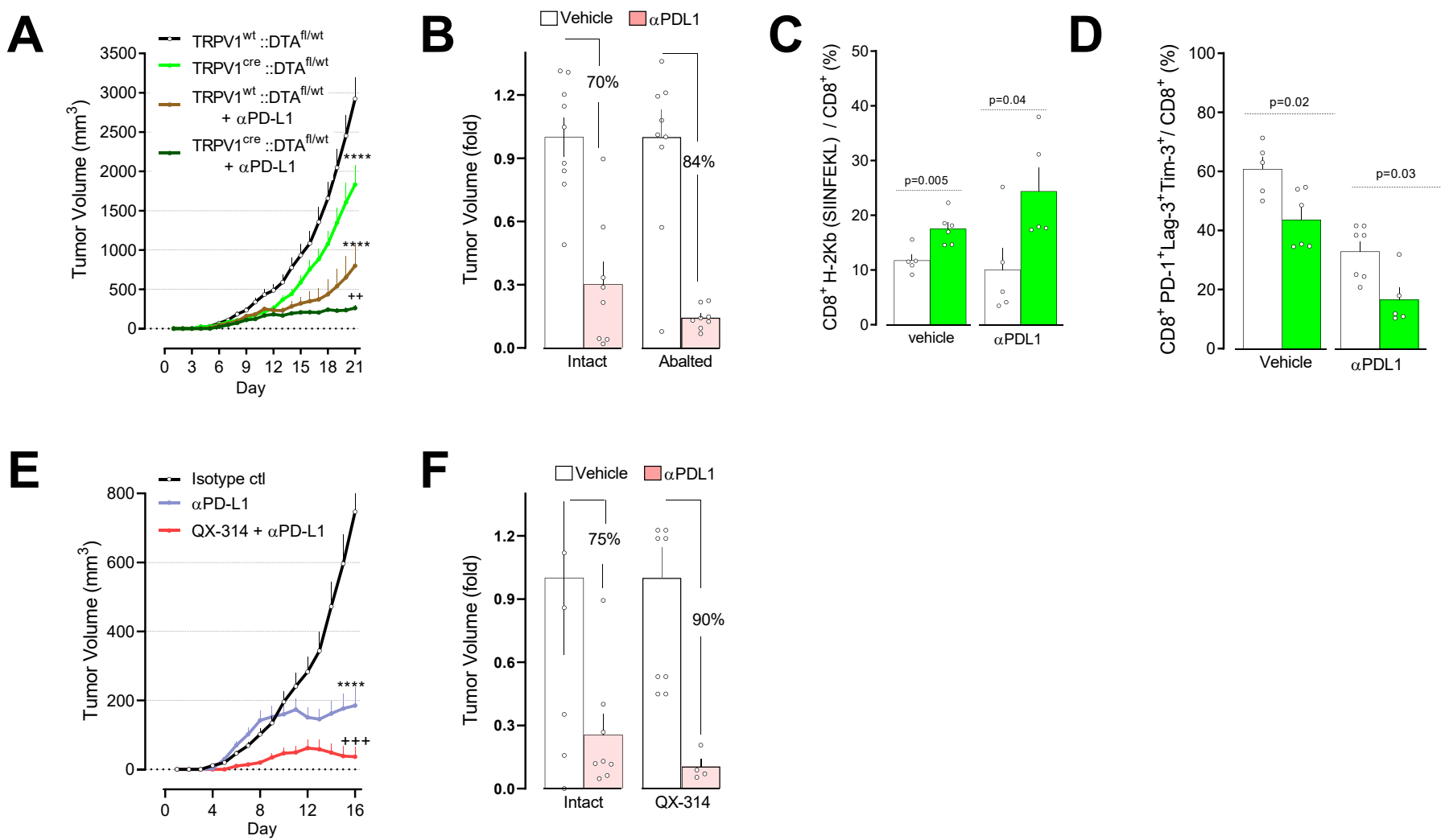

Supplementary Figure 36: aPDL1-mediated tumor regression is potentiated in absence of nociceptor neurons. Orthotopic B16F10-mCherry-OVA cells (500,000 cells; i.d.) were inoculated into 8-week-old male and female sensory neuron intact or ablated mice. On days 7, 10, and 13 post-tumor inoculation, the mice were treated with $\alpha \mathrm{PDL} 1$ (6 mg/kg, i.p.) or its vehicle (A-D). On day 21, we found that aPDL1 potentiated nociceptor ablation mediated reduction in B16F10-OVA tumor volume (A, raw value; $\mathbf{B}$, fold change vs control). Twenty-one days post-tumor inoculation, we found that nociceptor ablation increased the proportion of intra-tumoral tumor-specific $\left(\mathbf{C}\right.$; defined as $\mathrm{H}_{2} \mathrm{~KB}^{+}$) $\mathrm{CD}^{+} \mathrm{T}$-cells while it reduced their relative exhaustion (D, defined as PD1 ${ }^{+} \operatorname{Lag} 3^{+}{ }^{T i m} 3^{+}$). These differences were further enhanced by aPDL1 treatment (A-D). Orthotopic B16F10-mCherry-OVA cells (500,000 cells; i.d.) were inoculated into 8-week-old male and female mice. Starting one day postinoculation, a group of vehicle-exposed B16F10-bearing mice was injected with QX-314 (0.3\%; i.d., 5 sites around the tumor) once daily (E-F). On days 7, 10, and 13 post-tumor inoculation, the mice were exposed to aPDL1 (6 mg/kg, i.p.) or its vehicle. Sixteen days post-tumor inoculation, we found that nociceptor silencing potentiated aPDL1-mediated tumor reduction (E-F). Data are shown as mean \pm S.E.M; two-way ANOVA post hoc Bonferroni $(\boldsymbol{A}, \boldsymbol{E})$ or unpaired Student $t$-test (C-D); $p$-values are shown in the figure. 

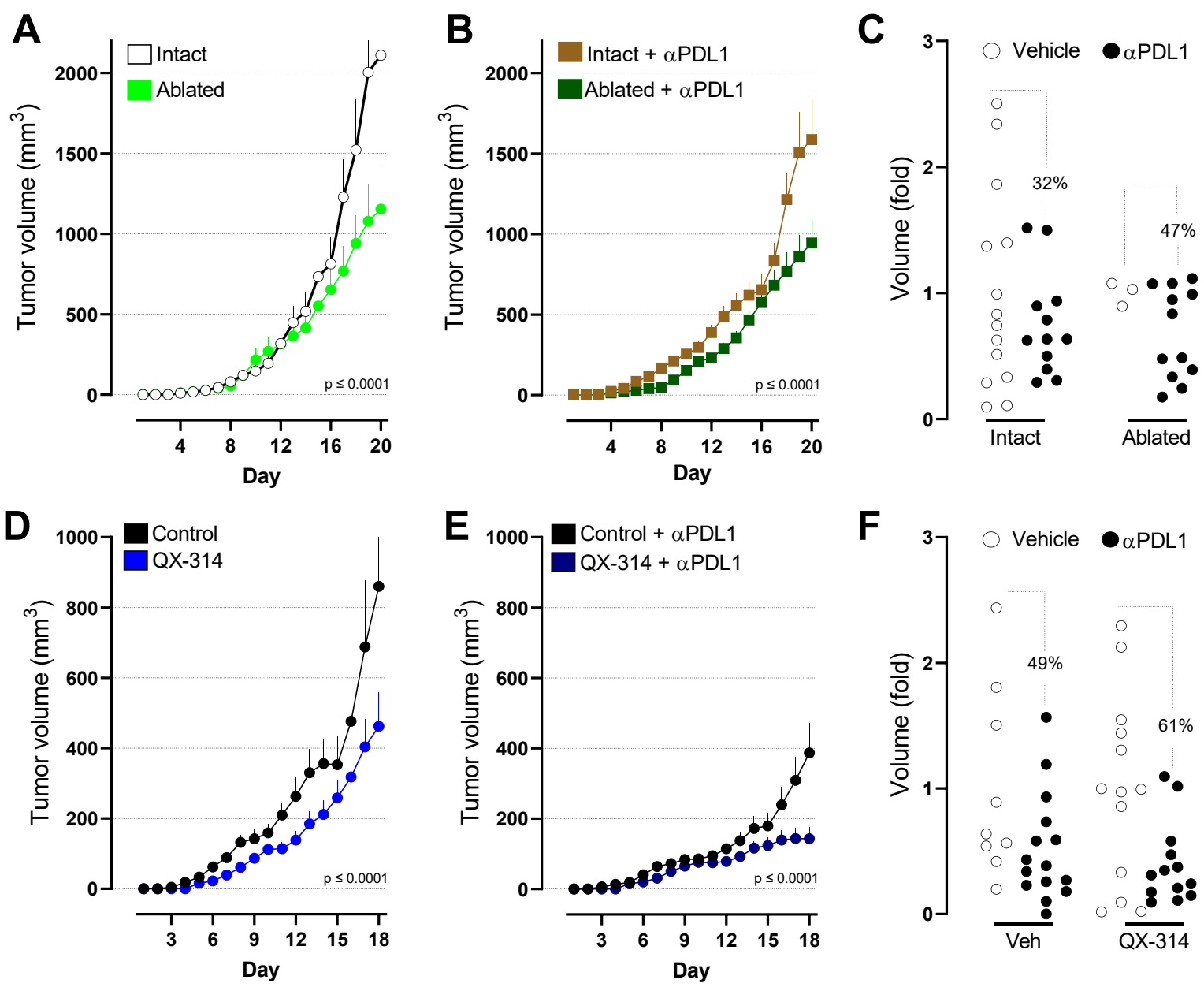

Supplementary Figure 37: Nociceptor neuron ablation or silencing potentiated aPDL1 reduction in tumor growth. Orthotropic B16F10-mCherry-OVA cells (500,000 cells, i.d.) were injected into a cohort of nociceptor neuron-ablated mice 3 days prior to one given to nociceptor intact animals. Mice from each group with similar tumor size $\left(\sim 85 \mathrm{~mm}^{3}\right)$ were selected and exposed to aPDL1 (6 mg/kg, i.p.) once every 3 days for a total of 9 days. Twenty days post-tumor inoculation, we found that aPDL1 reduced tumor growth was higher $(\sim 4 \%)$ in nociceptor-ablated mice than was observed in nociceptor intact mice ( 32\%; A-C). Orthotropic B16F10-mCherry-OVA cells $(500,000$ cells, i.d.) were injected into mice treated with QX-314 (0.3\%; i.d.) 2-3 days prior to being given to vehicle-exposed mice. Mice from each group with similar tumor size $\left(\sim 100 \mathrm{~mm}^{3}\right)$ were selected and exposed to aPDL1 (6 mg/kg, i.p.) once every 3 days for a total of 9 days. Eighteen days post-tumor inoculation, we found that aPDL1 reduced tumor growth was higher ( $61 \%)$ in nociceptor silenced mice than was observed in vehicleexposed mice ( 49\%; D-F). Data are shown as mean \pm S.E.M; two-way ANOVA (A, B, D, E); p-values are shown in the figure. 


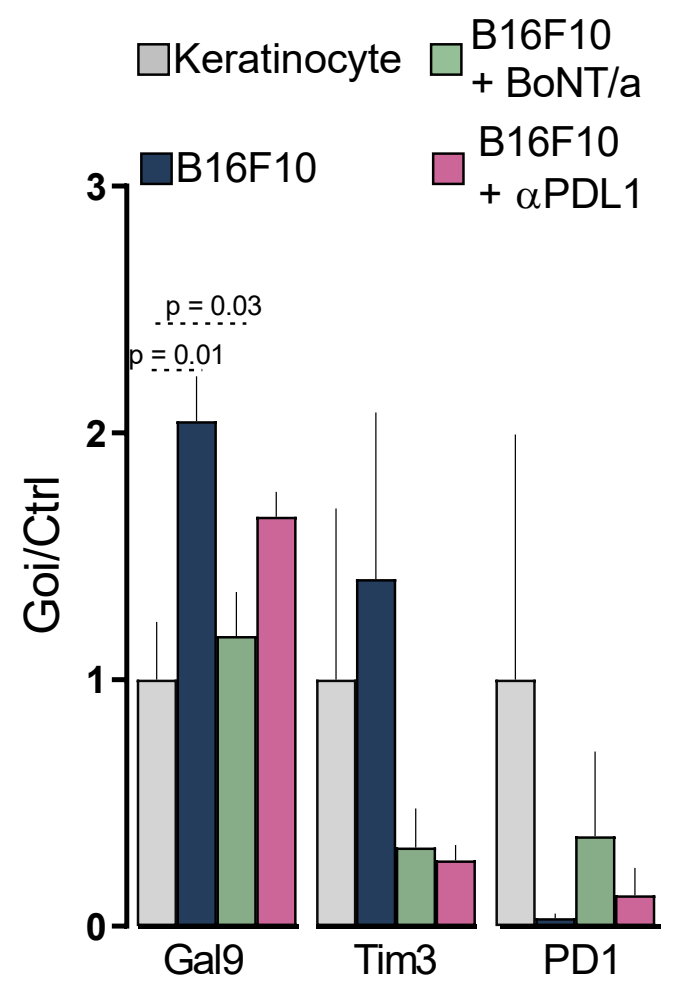

Supplementary Figure 38: Expression of PD1 decreased in tumor-innervating nociceptor neurons. One and three days

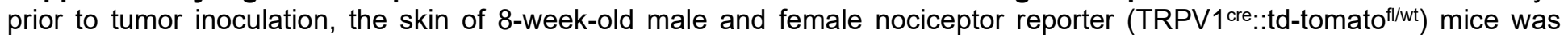
injected with BoNT/A (25 pg/ $\mathrm{\mu l}$; i.d.) or its vehicle. On the following day, orthotropic B16F10-mCherry-OVA cells (500,000 cells, i.d.) or non-tumorigenic keratinocytes (500,000 cells, i.d.) were inoculated into the area pre-exposed to BoNT/A. Starting one day post-inoculation, a group of vehicle-exposed B16F10-bearing mice was injected with QX-314 (0.3\%; i.d., 5 sites around the tumor) once daily. Fourteen days post-tumor inoculation, L3-L5 DRG neurons were harvested, TRPV1 ${ }^{+}$neurons were FACS-purified ( $\mathrm{Td}$-tomato ${ }^{+}$), and gene expression was measured by qPCR. We found that in comparison to TRPV $1^{+}$nociceptor neurons from keratinocytes-injected skin, FACS-sorted B16F10-infiltrating TRPV1 ${ }^{+}$nociceptor neurons expressed less Pd1 transcript. Data are shown as mean \pm S.E.M. one-way ANOVA post hoc Bonferroni. 
A

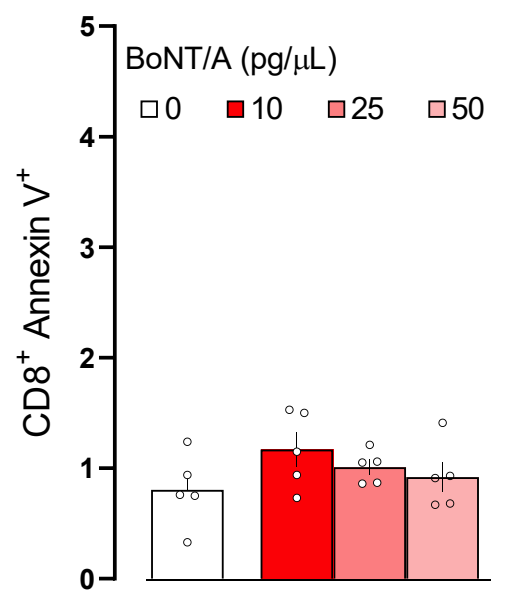

$\mathrm{B}$

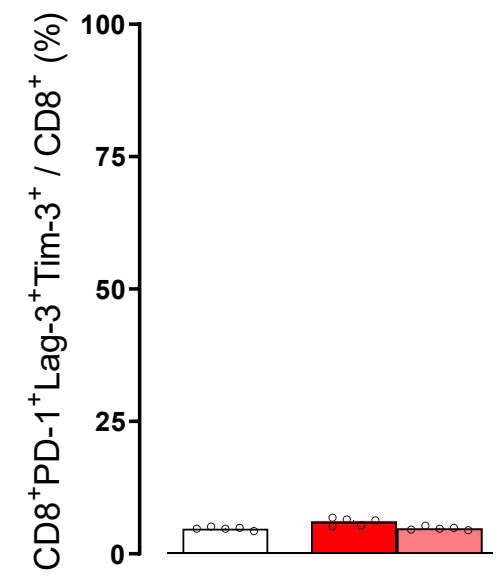

C
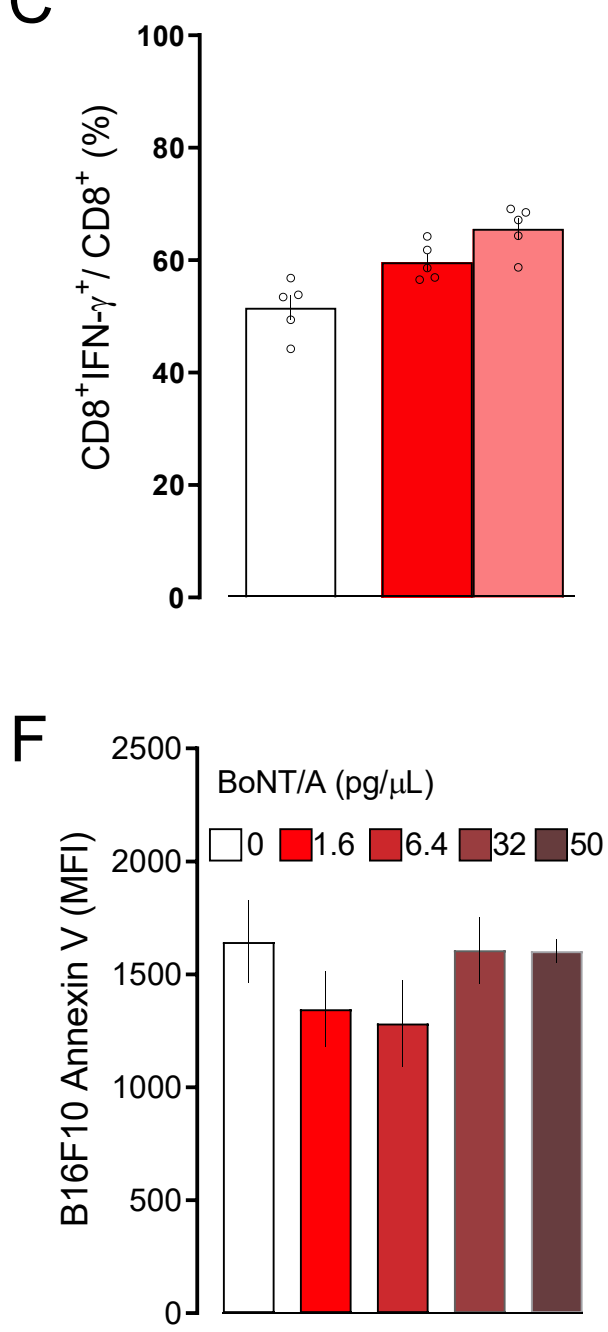

Supplementary Figure 39: Botox does not impact CD8 ${ }^{+} \mathrm{T}$-cells or B16F10 cell function. Splenocytes-isolated CD8 ${ }^{+} \mathrm{T}-$ cells from naive C57BL6 mice were cultured under Tc1-stimulating conditions for 48h. The cells were then exposed to BoNT/A (10-50 pg/ $/ \mathrm{L}$ ) for $24 \mathrm{~h}$, and effects on apoptosis, exhaustion, and activation were measured by flow cytometry. When compared to vehicle-exposed cells, BoNT/A did not impact the survival (A) of cultured cytotoxic CD8 ${ }^{+}$T-cells, nor their relative expression of $\mathrm{PD}^{+} \mathrm{Lag3}^{+} \mathrm{Tim3}^{+}(\mathbf{B}), \mathrm{IFNy}^{+}(\mathbf{C}), \mathrm{TNFa}^{+}$(D), and IL2+ ${ }^{+}$(E). B16F10 (100,000 cells) were cultured for $24 \mathrm{~h}$ and subsequently exposed to BoNT/A (1.6-50 pg/ $/ \mathrm{LL}$ ) or its vehicle for an additional $24 \mathrm{~h}$. BoNT/A did not trigger B16F10 cells apoptosis, as measured by the mean fluorescence intensity of Annexin $\vee(F)$. Data are shown as mean \pm S.E.M; one-way ANOVA post hoc Bonferroni. 

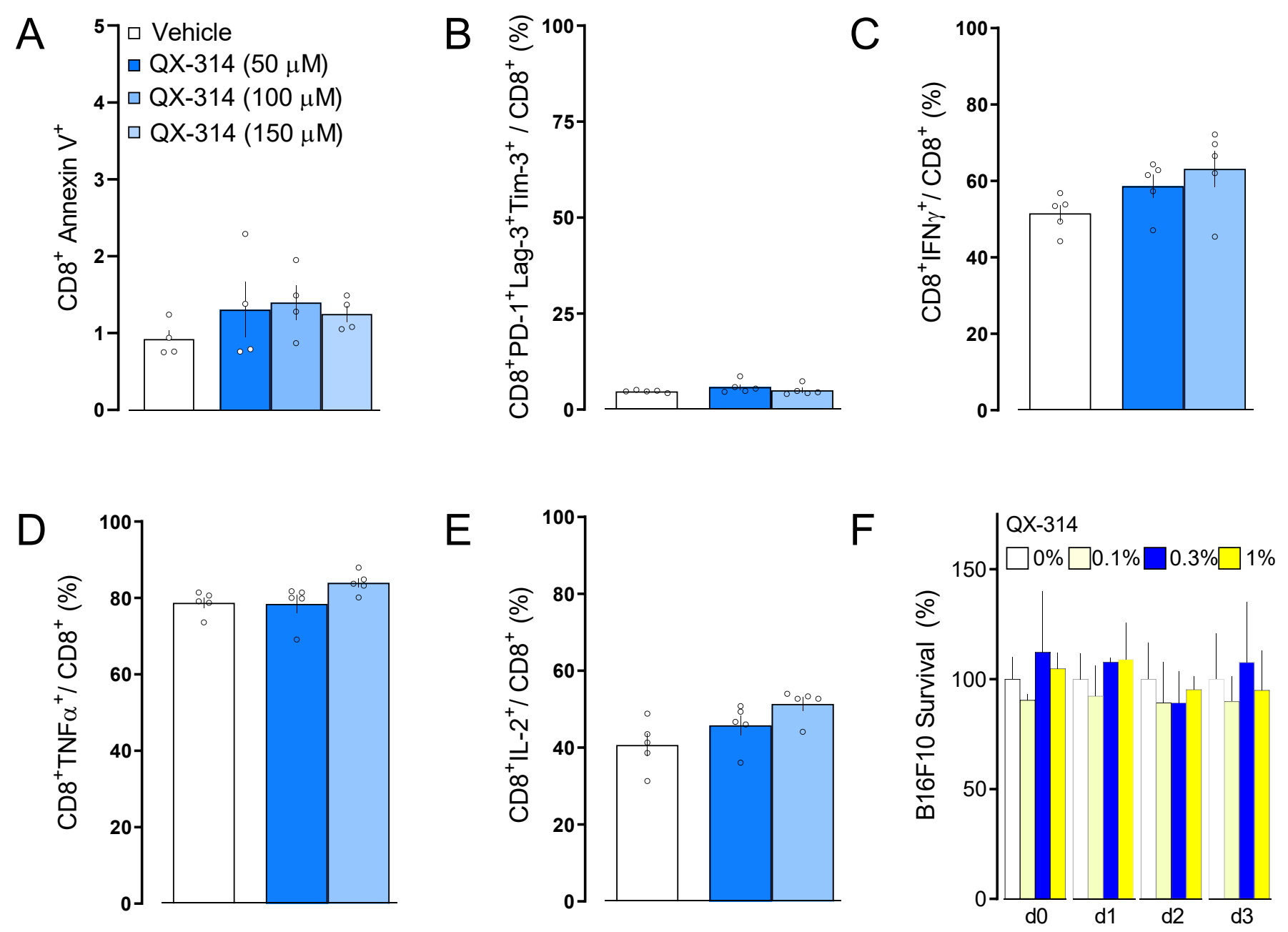

Supplementary Figure 40: QX-314 does not impact CD8 ${ }^{+}$T-cells or B16F10 cell function. Splenocytes-isolated CD8 ${ }^{+}$Tcells from naive C57BL6 mice were cultured under Tc1-stimulating conditions for 48h. The cells were then exposed to QX-314 (50-150 $\mu \mathrm{M})$ for $24 \mathrm{~h}$, and effects on apoptosis, exhaustion and activation were measured by flow cytometry. When compared to vehicle-exposed cells, QX-314 did not impact the survival (A) of cultured cytotoxic CD8 ${ }^{+}$T-cells, nor their relative expression of PD1 ${ }^{+} \mathrm{Lag}^{+} \mathrm{Tim}^{+}$(B), IFNY ${ }^{+}$(C), TNFa ${ }^{+}$(D), and IL2 ${ }^{+}$(E). B16F10 $(100,000$ cells) were cultured for $24 \mathrm{~h}$. The cells were then exposed or not to QX-314 (0.1-1\%) for an additional 24-72h, and cell count was analyzed by bright-field microscopy. QX-314 did not impact B16F10 cells' survival, as measured by relative cell count changes (at each time point) in comparison to vehicleexposed cells $(\mathbf{F})$. Data are shown as mean \pm S.E.M; one-way ANOVA post hoc Bonferroni. 

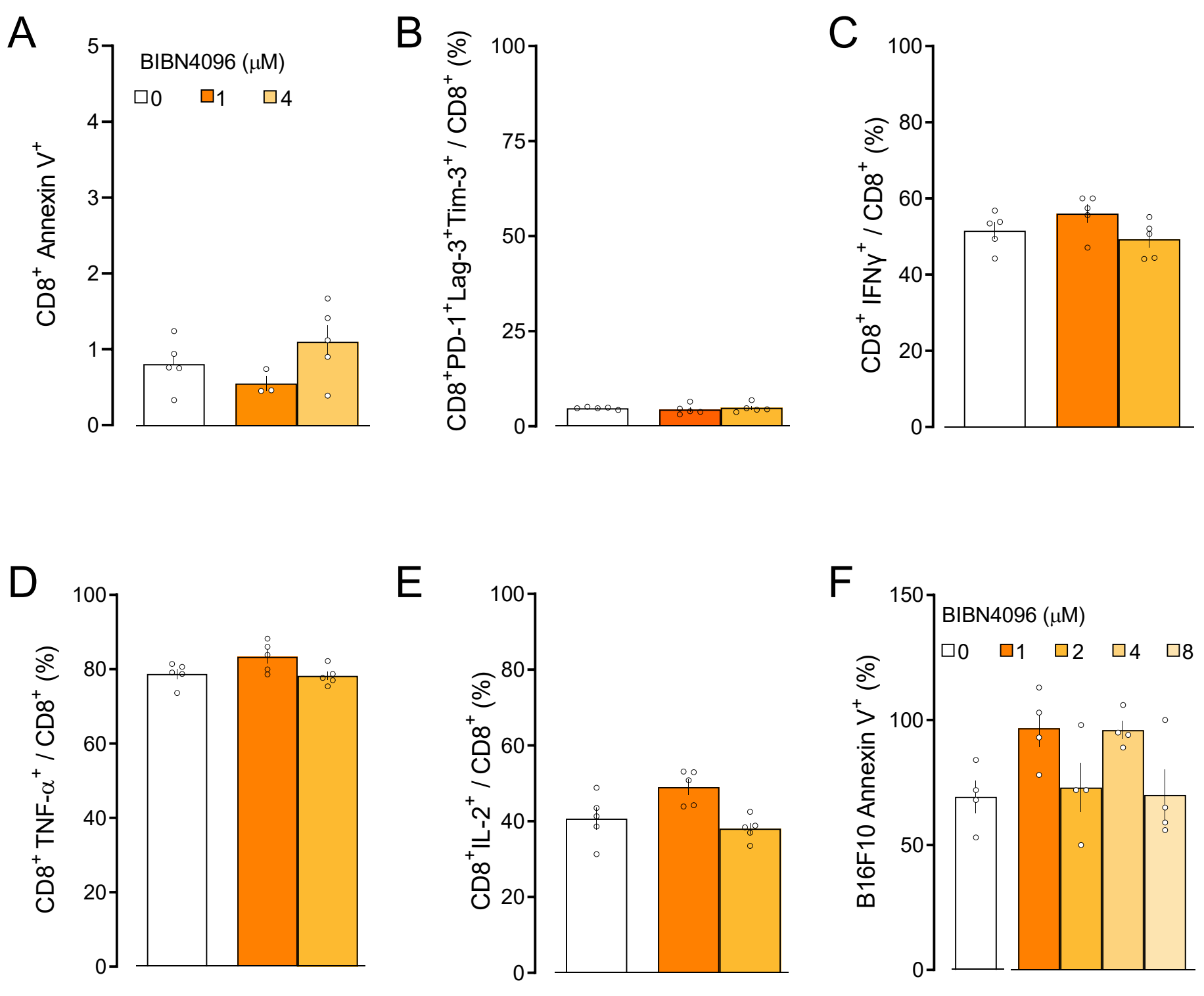

Supplementary Figure 41: BIBN4096 does not impact CD8 ${ }^{+}$T-cell function. Splenocytes-isolated CD8 ${ }^{+}$T-cells from naive C57BL6 mice were cultured under Tc1-stimulating conditions for 48h. The cells were then exposed to BIBN4096 (1-4 $\mu \mathrm{M})$ for $24 \mathrm{~h}$, and effects on apoptosis, exhaustion and activation were measured by flow cytometry. When compared to vehicleexposed cells, BIBN4096 did not impact the survival (A) of cultured cytotoxic CD8 ${ }^{+}$T-cells, nor their relative expression of

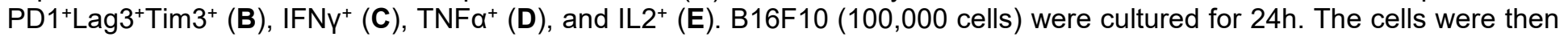
exposed or not to BIBN4096 (1-4 $\mu \mathrm{M})$ for an additional $24 \mathrm{~h}$, and effects on apoptosis were measured by flow cytometry. BIBN4096 did not trigger B16F10 cells apoptosis, as measured by the mean fluorescence intensity of Annexin $V(\mathbf{F})$. Data are shown as mean \pm S.E.M; one-way ANOVA post hoc Bonferroni. 
A
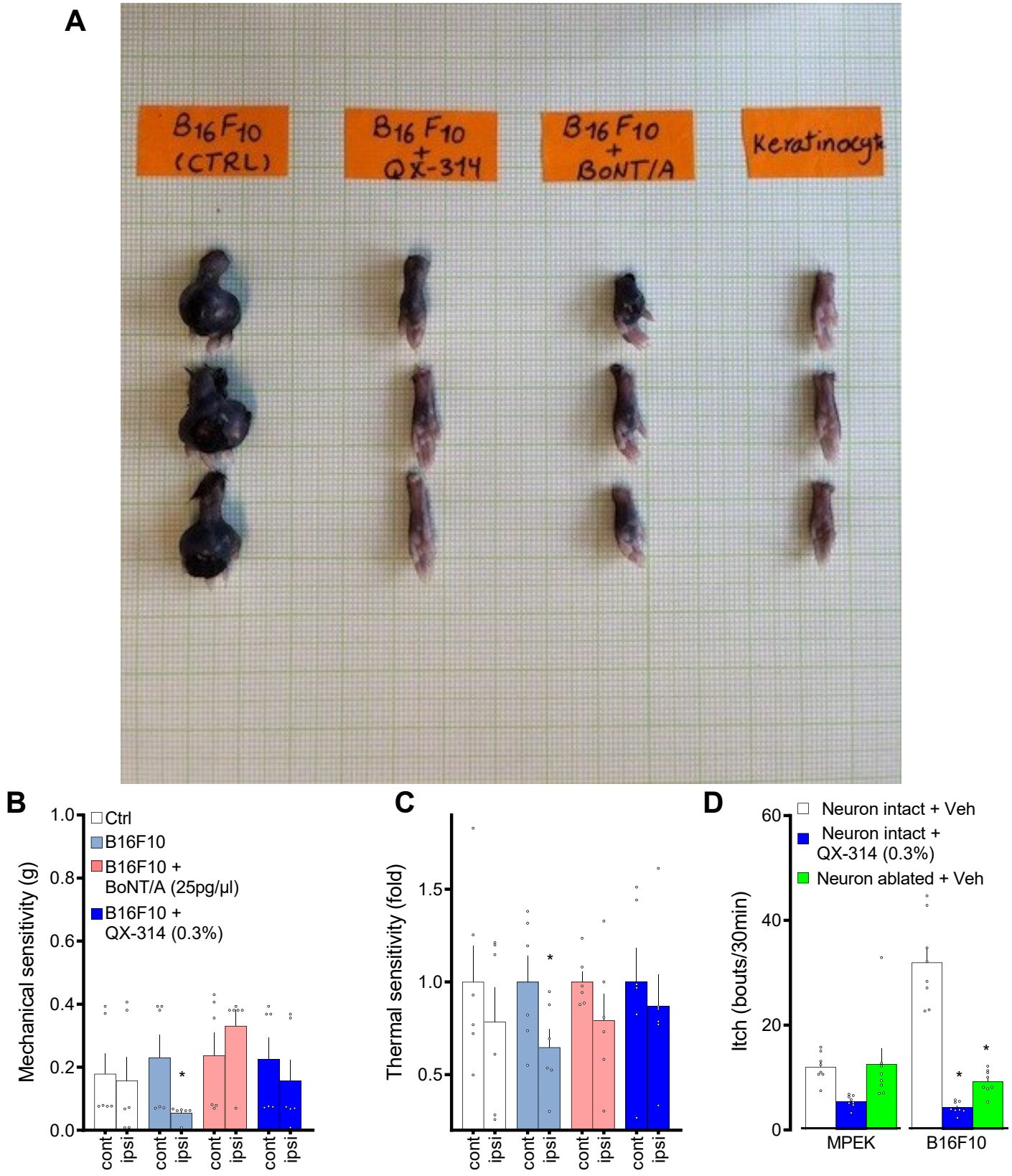

Supplementary Figure 42: Sensory neuron silencing prevents tumor-induced pain and itch. One and three days prior to tumor inoculation, 8-week-old male and female sensory neuron intact mice's right hind paws were injected with BoNT/A (25 $\mathrm{pg} / \mathrm{\mu l}$; i.d.) or its vehicle. On the following day, orthotopic B16F10 cells $(200,000$ cells; i.d.) were inoculated into the area preexposed to BoNT/A. On a different group of mice, orthotopic B16F10-mCherry-OVA cells $(200,000$ cells; i.d.) or nontumorigenic keratinocytes (200,000 cells; i.d.; labeled as MPEK) were inoculated into 8-week-old male and female sensory neuron intact (TRPV1 ${ }^{\mathrm{wt}}::$ DTA ${ }^{\mathrm{f} / \mathrm{wt}}$ ) and ablated (TRPV1 ${ }^{\left.\text {cre }:: D T A^{\mathrm{f} / \mathrm{wt}}\right)}$ mice's right hind paws. Starting one day post-inoculation, QX-314 (0.3\%) or its vehicle was administered (i.p.) once daily in a group of littermate control mice. The effects of sensory neuron silencing or ablation were tested on mechanical and thermal pain hypersensitivity. Fourteen days post tumor inoculation, we found that B16F10 injection induced tumor formation (A), mechanical (B) and thermal pain hypersensitivities (C), while flank injection triggered occasional ( $\sim 30 \%)$ itch (D). These effects were stopped by sensory neuron silencing (QX-314; A-D) and BoNT/A (A-C) or absent in mice whose sensory neurons were genetically ablated (D). Data are shown as mean \pm S.E.M; unpaired Student t-test (B-C) or one-way ANOVA post hoc Bonferroni (D). 


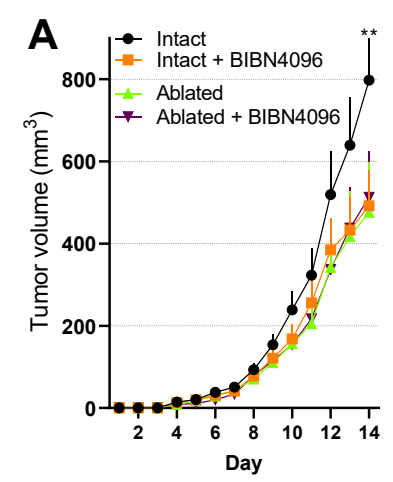

D
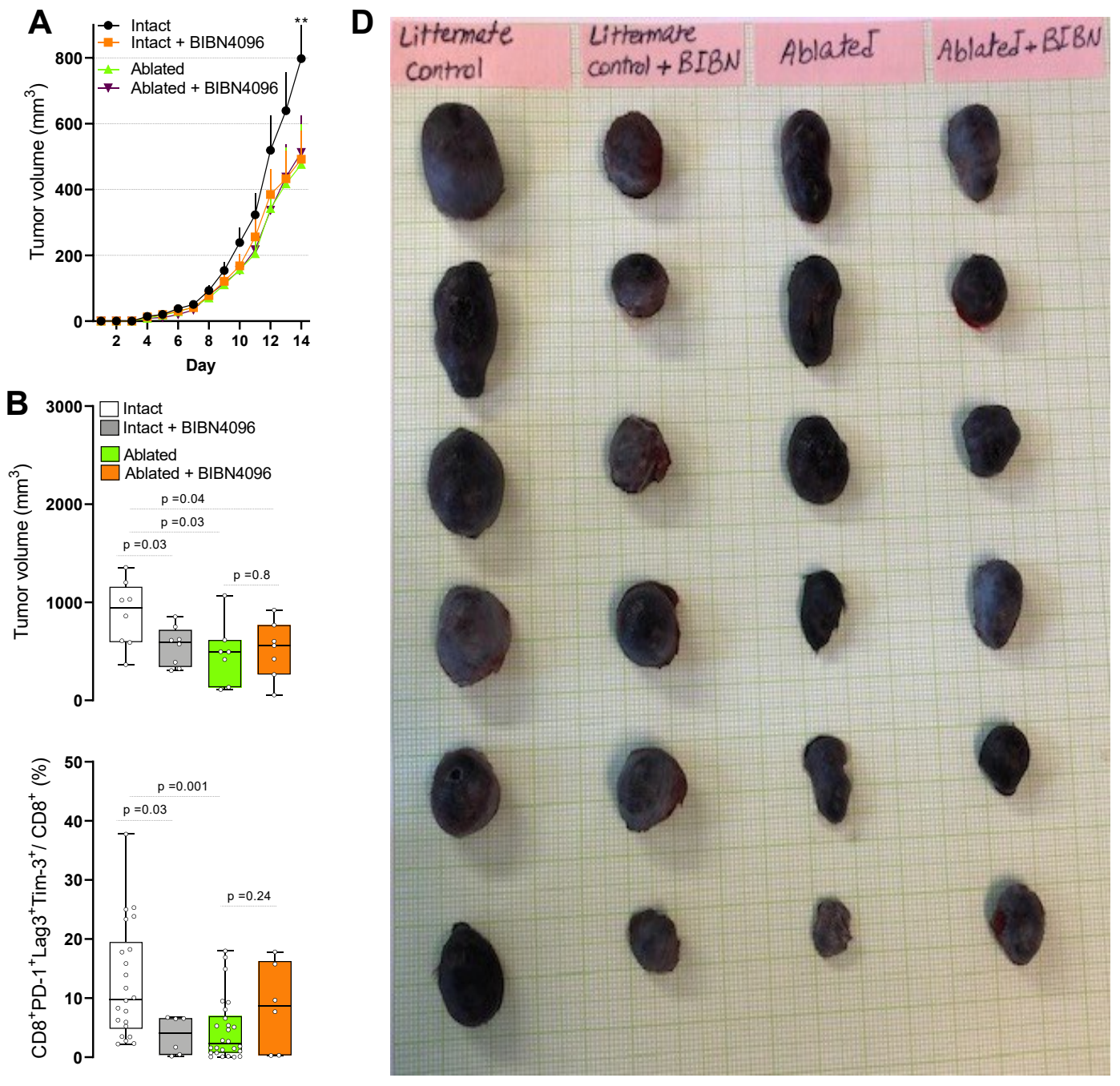

C

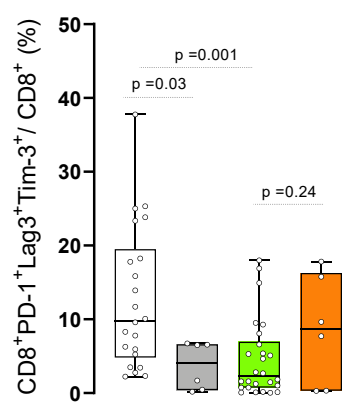

Supplementary Figure 43: RAMP1 antagonism did not impact tumor growth when administered to sensory neuronablated mice. Orthotopic B16F10-mCherry-OVA cells (500,000 cells; i.d.) were inoculated into 8-week-old male and female

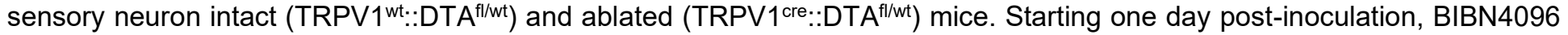
$(5 \mathrm{mg} / \mathrm{kg})$ or its vehicle was administered (i.p.) on alternate days and effects on tumor size and tumor-infiltrating CD8 ${ }^{+} \mathrm{T}_{\text {-cells' }}$ relative exhaustion was measured. Fourteen days post tumor inoculation, we found that the tumor growth $(\mathbf{A})$, volume (B), and size (D), as well as the relative expression of $\mathrm{PD} 1^{+} \mathrm{Lag}^{+}{ }^{+} \mathrm{Tim} 3^{+}$in tumor-infiltrating $\mathrm{CD}^{+}{ }^{+} \mathrm{T}$-cells $(\mathbf{C})$, were reduced in sensory neuron-ablated mice or in BIBN4096-treated mice. BIBN4096 had no additive effect when given to sensory neuron-ablated mice (A-D). Data are shown as mean \pm S.E.M; two-way (A) or one-way (B-C) ANOVA post hoc Bonferroni; $p$-values are shown in the figure. 


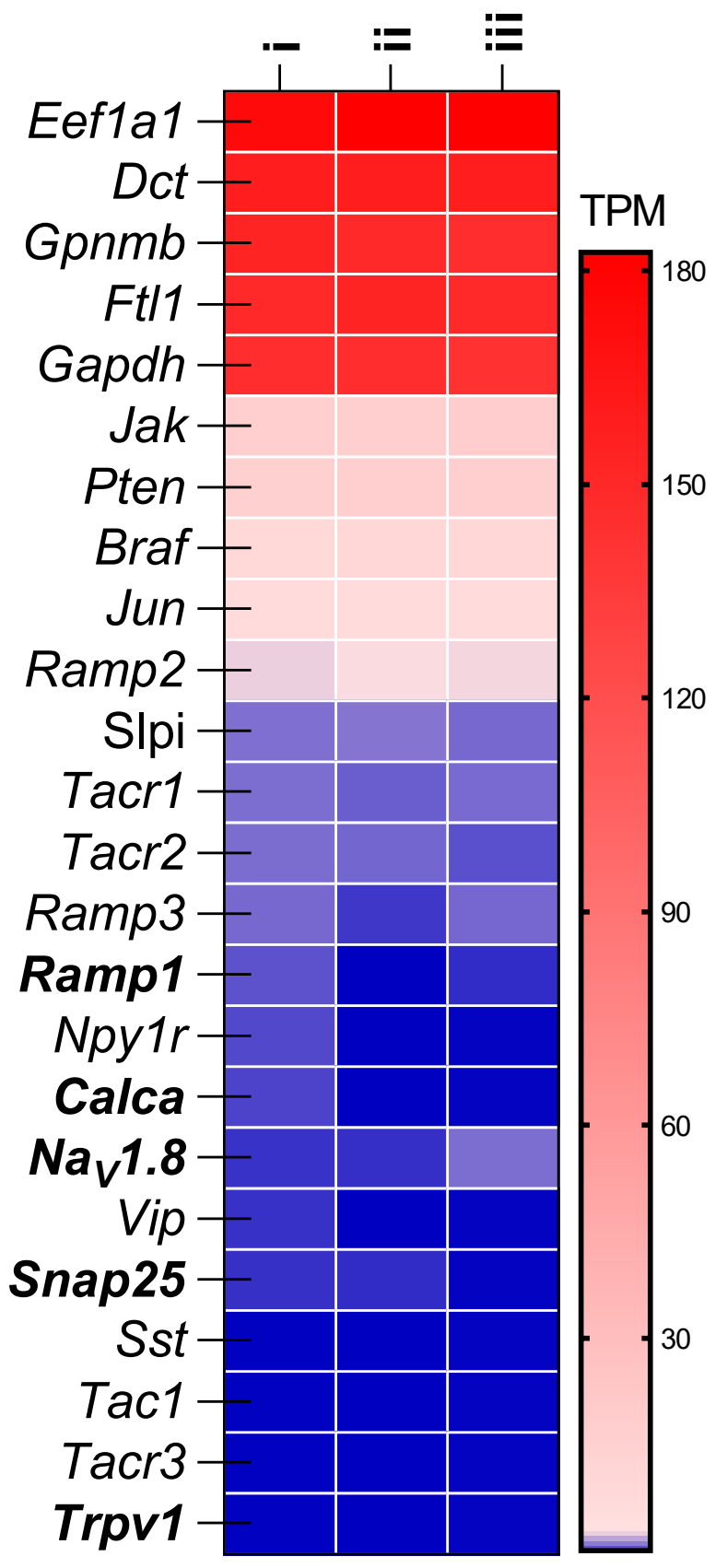

Supplementary Figure 44: B16F10 cells do not express Trpv1, Nav1.8, Snap25, or Ramp1. In-silico analysis of three different B16F10 cells cultures (labelled as $i$, ii, iii) ${ }^{71}$ revealed their basal expression of Braf and Pten. In contrast, B16F10 cells do not express Trpv1 or Nav1.8 (necessary for QX-314 activity), Snap25 (molecular target of BoNT/A), or Ramp1 (molecular target of BIBN4096). Heatmaps data are shown as transcript per million (TPM) on a linear scale. Experimental details were defined in Castle et al. ${ }^{71}$. 


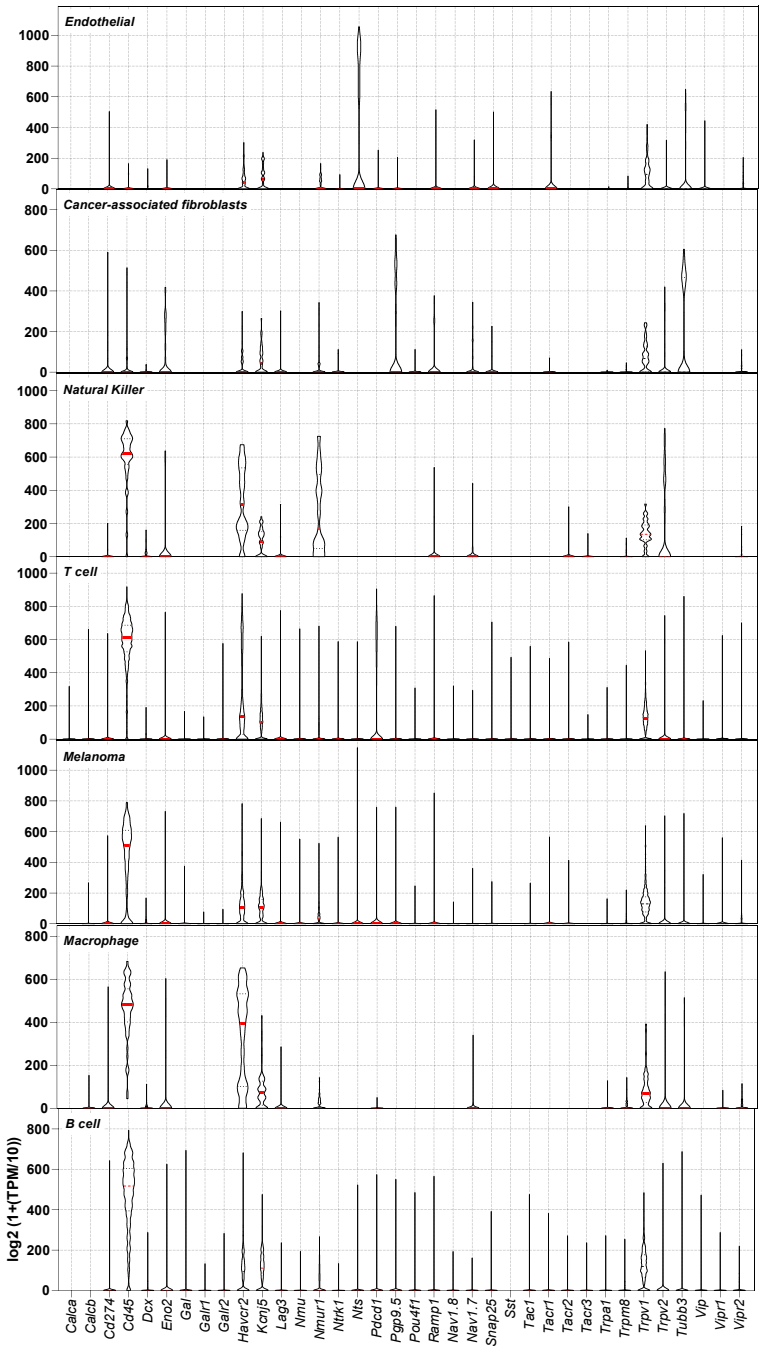

Supplementary Figure 45: Patients' melanoma stromal and infiltering leukocytes do not express Calca, Trpv1, or Snap25. In-silico analysis of single-cell RNA sequencing of human melanoma revealed that cancer-associated fibroblasts, macrophage, endothelial, natural killer, $T$, and B cells as well as malignant cells did not express Calca (gene encoding for CGRP), Snap25 (the molecular target of BoNT/A), or Trpv1 and Nav1.8 channels (essential for QX-314 activity). Individual cell data are shown as a $\log _{2}$ of $1+$ (transcript per million / 10). Experimental details and cell clustering were defined in Tirosh et al. ${ }^{45}$ 


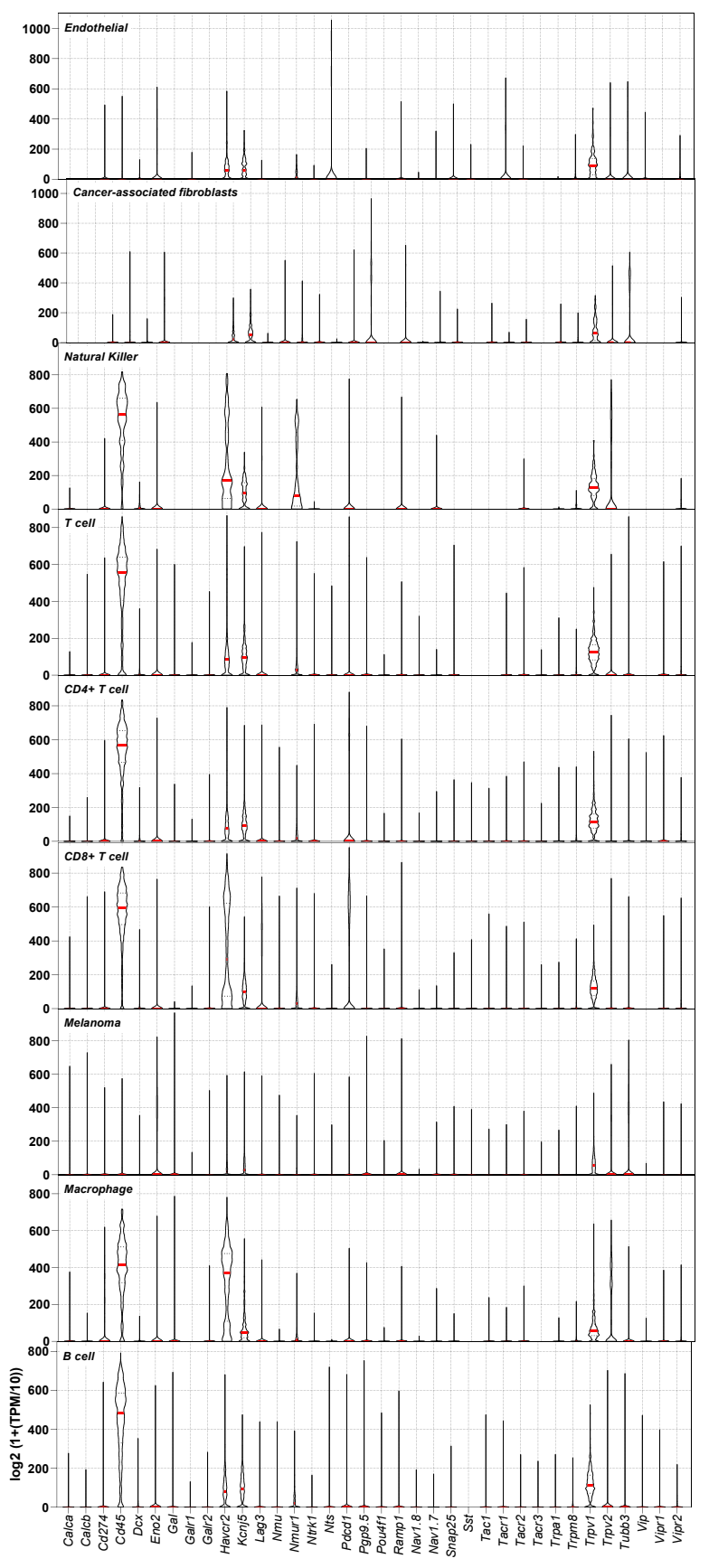

Supplementary Figure 46: Patients' biopsies revealed that Calca, Trpv1, and Snap25 are solely expressed by nociceptors. In-silico analysis of single-cell RNA sequencing of human melanoma-infiltrating cells revealed that cancerassociated fibroblasts, macrophage, endothelial, natural killer, $\mathrm{T}$, and $\mathrm{B}$ cells as well as malignant cells do not express Calca (gene encoding for CGRP), Snap25 (the molecular target of BoNT/A), or Trpv1 and Nav1.8 channels (essential for QX-314 activity). Individual cell data are shown as a $\log _{2}$ of $1+$ (transcript per million / 10). Experimental details and cell clustering were defined in Jerby-Arnon et al. ${ }^{4}$ 


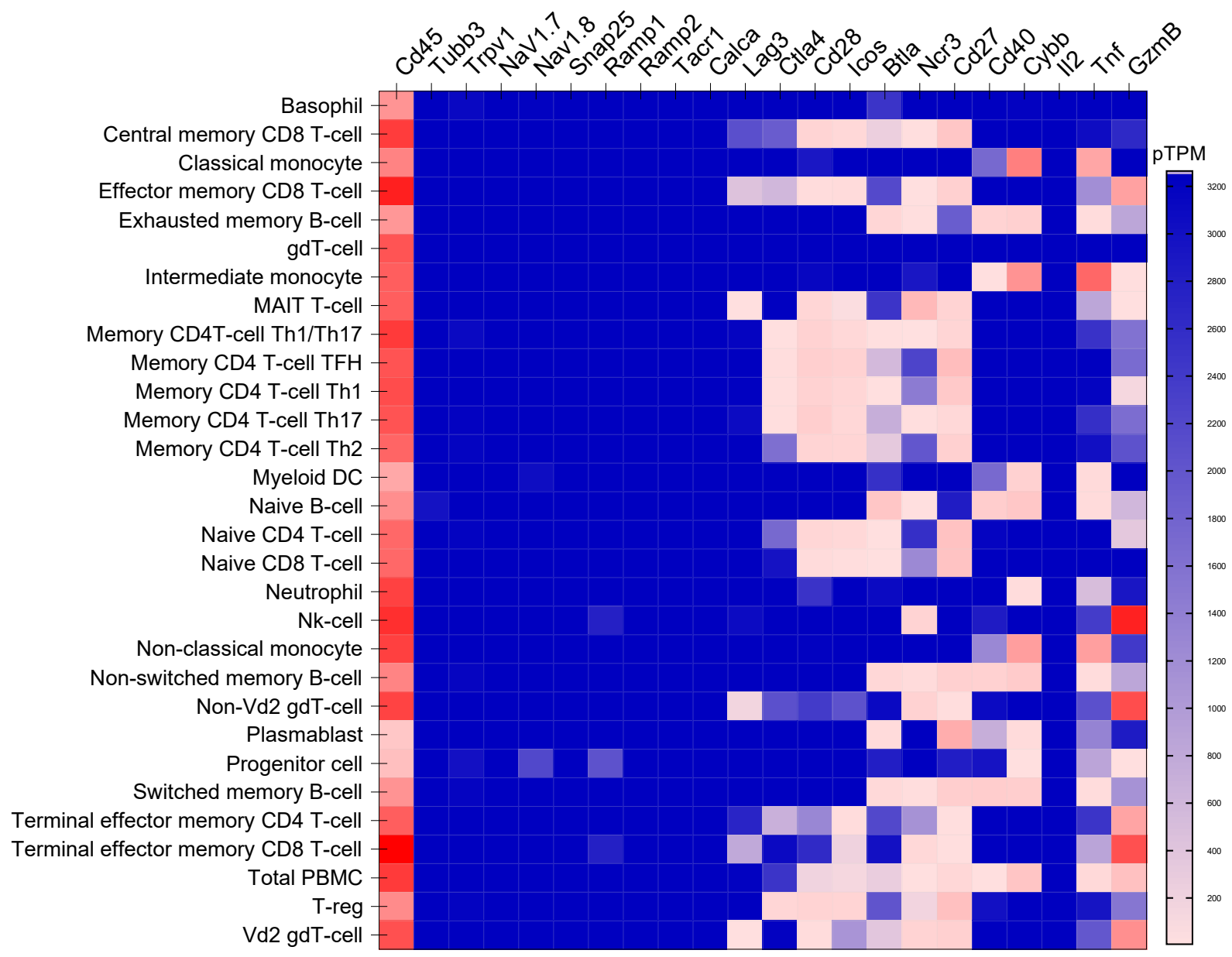

Supplementary Figure 47: Human immunocytes do not express Calca, Trpv1, Nav1.7, or Snap25. In-silico analysis of human immunocytes revealed their basal expression of $C d 45$ and their absence of expression of Calca (gene encoding for CGRP), Snap25 (the molecular target of BoNT/A), or Trpv1 and Nav1.8 channels (essential for QX-314 activity). Heatmaps show the read counts normalized to transcripts per million protein-coding genes (pTPM) for each of the single-cell clusters. Experimental details and cell clustering were defined in Monaco et al. ${ }^{72}$ 


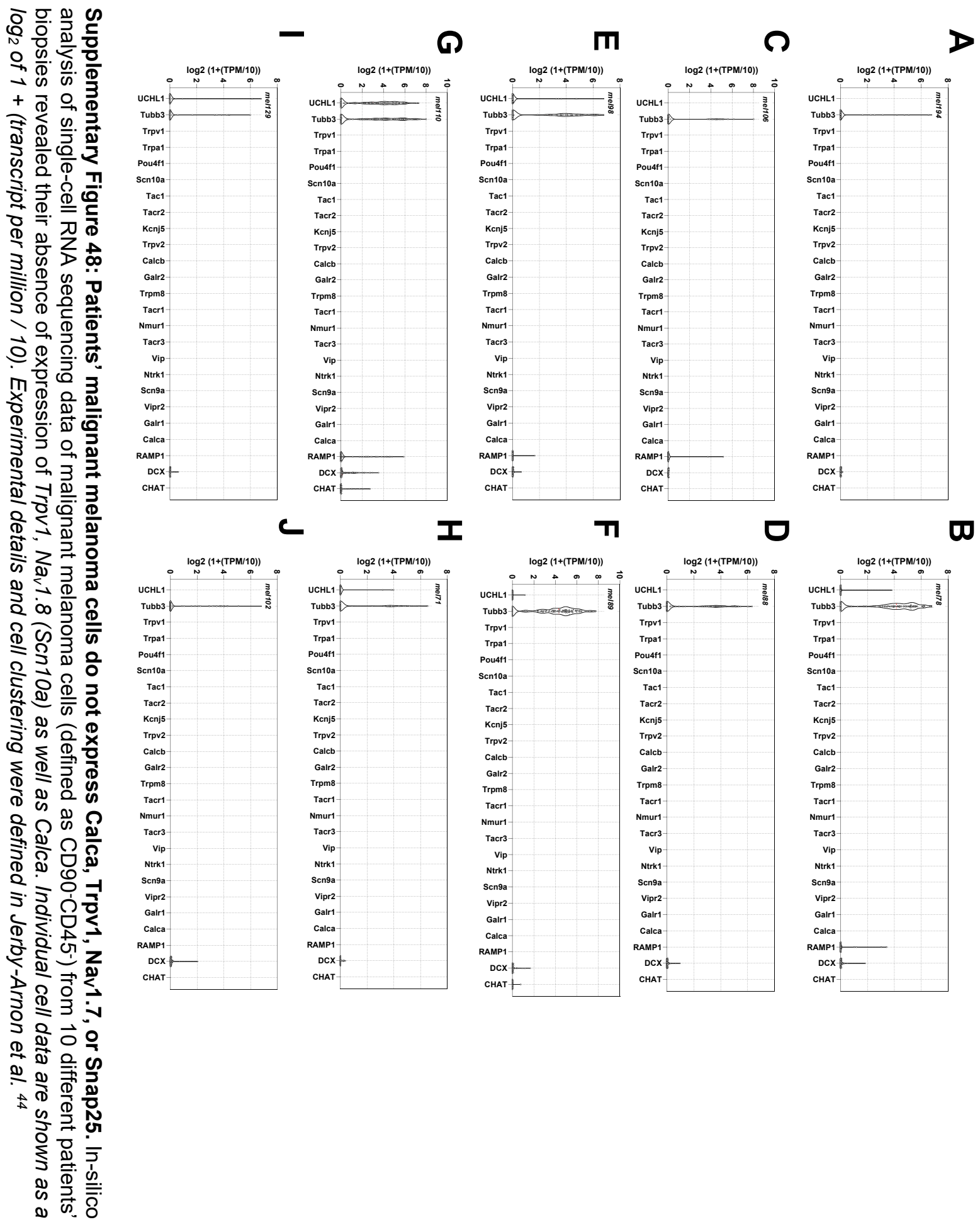



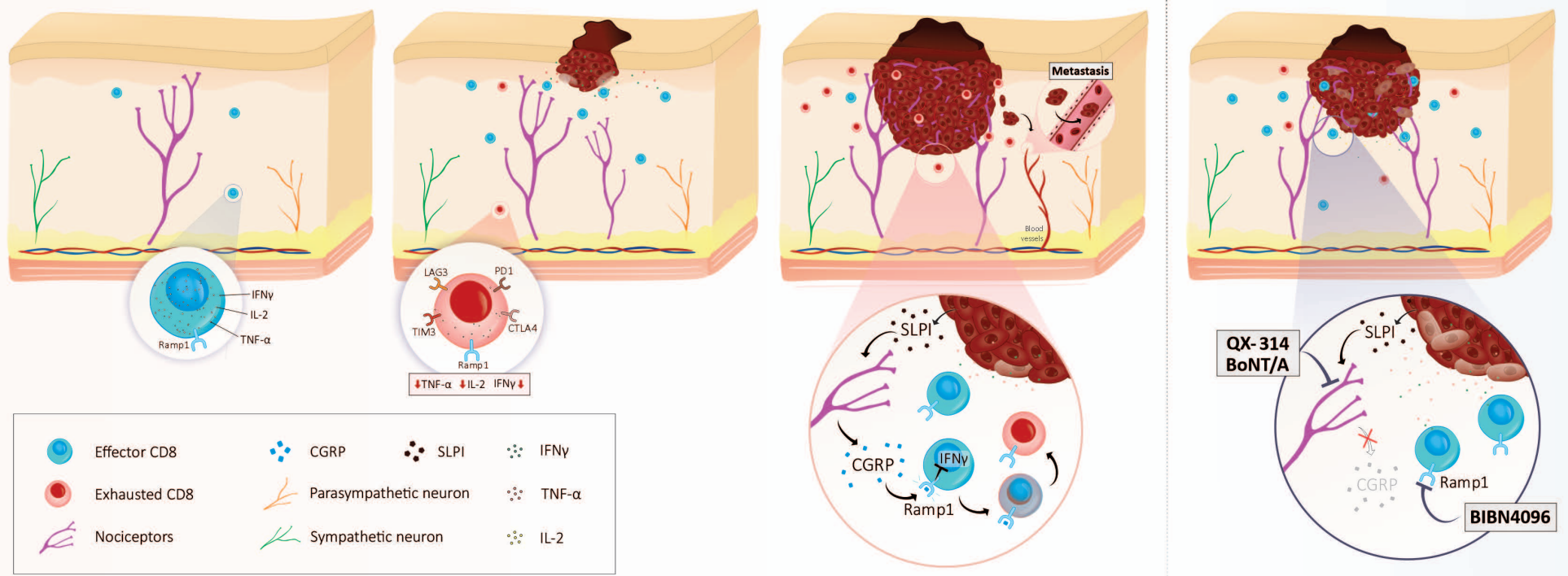

Supplementary Figure 49: Melanoma-innervating nociceptors impair cancer immunosurveillance. Melanoma growth sets off anti-tumor immune responses, including the infiltration of effector CD8 T-cells and their subsequent release of cytotoxic cytokines (i.e., IFNY, TNFa, Granzyme B). By acting on tissue-resident nociceptor neurons, melanoma-produced SLPI promotes pain hypersensitivity, tweaks the neurons' transcriptome, and drives neurite outgrowth. These effects culminate in dense melanoma innervation by nociceptors and abundant release of immunomodulatory neuropeptides. CGRP, one such peptide, acts on tumor-infiltrating RAMP1 ${ }^{+}$effector CD8 T-cells, increasing their expression of immune checkpoint receptors (i.e., PD1, LAG3, TIM3). Thus, along with the immunosuppressive environment present in the tumor, nociceptor-produced CGRP leads to the functional exhaustion of tumor-infiltrating CD8 T-cells; leaving unchecked, melanoma can proliferate. Genetically ablating (i.e., NaV1.8 or TRPV1 lineage) or pharmacologically silencing (i.e., QX-314, BoNT/A) nociceptor neurons as well as blocking the action of CGRP on RAMP1 using a selective antagonist (i.e., BIBN4096) prevents effector CD8 T-cells from undergoing exhaustion. Therefore, targeting melanoma-innervating nociceptor neurons constitutes a novel strategy to safeguard the host anti-tumor immunity and stop tumor growth. 


\section{References}

1 Mauffrey, P. et al. Progenitors from the central nervous system drive neurogenesis in cancer. Nature 569, 672-678, doi:10.1038/s41586-019-1219-y (2019).

2 Magnon, C. et al. Autonomic nerve development contributes to prostate cancer progression. Science 341, 1236361, doi:10.1126/science.1236361 (2013).

3 Zahalka, A. H. et al. Adrenergic nerves activate an angio-metabolic switch in prostate cancer. Science 358, 321-326, doi:10.1126/science.aah5072 (2017).

4 Zahalka, A. H. \& Frenette, P. S. Nerves in cancer. Nat Rev Cancer 20, 143-157, doi:10.1038/s41568-019-0237-2 (2020).

5 Amit, M. et al. Loss of p53 drives neuron reprogramming in head and neck cancer. Nature 578, 449-454, doi:10.1038/s41586-020-1996-3 (2020). 6 Chambers, C. A., Kuhns, M. S., Egen, J. G. \& Allison, J. P. CTLA-4-mediated inhibition in regulation of T cell responses: doi:10.1146/annurev.immunol.19.1.565 (2001).

7 Das, M., Zhu, C. \& Kuchroo, V. K. Tim-3 and its role in regulating anti-tumor immunity. Immunol Rev 276, 97-111, doi:10.1111/imr.12520 (2017).

8 Dougan, M. \& Dranoff, G. Immune therapy for cancer. Annu Rev Immunol 27, 83-117, doi:10.1146/annurev.immunol.021908.132544 (2009).

9 Topalian, S. L. et al. Safety, activity, and immune correlates of anti-PD-1 antibody in cancer. N Engl J Med 366, 24432454, doi:10.1056/NEJMoa1200690 (2012).

Baumeister, S. H., Freeman, G. J., Dranoff, G. \& Sharpe, A. H. Coinhibitory Pathways in Immunotherapy for Cancer. Annu Rev Immunol 34, 539-573, doi:10.1146/annurev-immunol-032414-112049 (2016).

11 Vesely, M. D., Kershaw, M. H., Schreiber, R. D. \& Smyth, M. J. Natural innate and adaptive immunity to cancer. Annu Rev Immunol 29, 235-271, doi:10.1146/annurev-immunol-031210-101324 (2011).

12 Woo, S. R., Corrales, L. \& Gajewski, T. F. Innate immune recognition of cancer. Annu Rev Immunol 33, 445-474, doi:10.1146/annurev-immunol-032414-112043 (2015).

13 Anderson, P. \& Gonzalez-Rey, E. Vasoactive intestinal peptide induces cell cycle arrest and regulatory functions in human T cells at multiple levels. Mol Cell Biol 30, 2537-2551, doi:10.1128/MCB.01282-09 (2010).

14 Chen, G. et al. PD-L1 inhibits acute and chronic pain by suppressing nociceptive neuron activity via PD-1. Nat Neurosci 20, 917-926, doi:10.1038/nn.4571 (2017).

15 Kamiya, A. et al. Genetic manipulation of autonomic nerve fiber innervation and activity and its effect on breast cancer progression. Nat Neurosci 22, 1289-1305, doi:10.1038/s41593-019-0430-3 (2019).

$16 \mathrm{Li}, \mathrm{J}$. M. et al. VIPhyb, an antagonist of vasoactive intestinal peptide receptor, enhances cellular antiviral immunity in murine cytomegalovirus infected mice. PLoS One 8, e63381, doi:10.1371/journal.pone.0063381 (2013).

$\mathrm{Li}$, J. M. et al. Modulation of Immune Checkpoints and Graft-versus-Leukemia in Allogeneic Transplants by Antagonizing Vasoactive Intestinal Peptide Signaling. Cancer Res 76, 6802-6815, doi:10.1158/0008-5472.CAN-160427 (2016).

18 Ondicova, K. \& Mravec, B. Role of nervous system in cancer aetiopathogenesis. Lancet Oncol 11, 596-601, doi:10.1016/S1470-2045(09)70337-7 (2010).

Petersen, C. T., Li, J. M. \& Waller, E. K. Administration of a vasoactive intestinal peptide antagonist enhances the autologous anti-leukemia T cell response in murine models of acute leukemia. Oncoimmunology 6, e1304336, doi:10.1080/2162402X.2017.1304336 (2017).

20 Pozo, D., Anderson, P. \& Gonzalez-Rey, E. Induction of alloantigen-specific human T regulatory cells by vasoactive intestinal peptide. J Immunol 183, 4346-4359, doi:10.4049/jimmunol.0900400 (2009).

21 Saloman, J. L. et al. Ablation of sensory neurons in a genetic model of pancreatic ductal adenocarcinoma slows initiation and progression of cancer. Proc Natl Acad Sci U S A 113, 3078-3083, doi:10.1073/pnas.1512603113 (2016). Talbot, S. et al. Silencing Nociceptor Neurons Reduces Allergic Airway Inflammation. Neuron 87, 341-354, doi:10.1016/j.neuron.2015.06.007 (2015).

23 Tebas, P. et al. Reduction of soluble CD163, substance P, programmed death 1 and inflammatory markers: phase 1B trial of aprepitant in HIV-1-infected adults. AIDS 29, 931-939, doi:10.1097/QAD.0000000000000638 (2015).

24 Huang, S. et al. Lymph nodes are innervated by a unique population of sensory neurons with immunomodulatory potential. Cell 184, 441-459 e425, doi:10.1016/j.cell.2020.11.028 (2021).

Renier, N. et al. iDISCO: a simple, rapid method to immunolabel large tissue samples for volume imaging. Cell 159, 896-910, doi:10.1016/j.cell.2014.10.010 (2014).

26 Goswami, S. C. et al. Molecular signatures of mouse TRPV1-lineage neurons revealed by RNA-Seq transcriptome analysis. J Pain 15, 1338-1359, doi:10.1016/j.jpain.2014.09.010 (2014). 
Hannila, S. S. et al. Secretory leukocyte protease inhibitor reverses inhibition by CNS myelin, promotes regeneration in the optic nerve, and suppresses expression of the transforming growth factor-beta signaling protein Smad2. J Neurosci 33, 5138-5151, doi:10.1523/JNEUROSCI.5321-12.2013 (2013).

Mueller, A. M. et al. Novel role for SLPI in MOG-induced EAE revealed by spinal cord expression analysis. $J$ Neuroinflammation 5, 20, doi:10.1186/1742-2094-5-20 (2008).

Baral, P. et al. Nociceptor sensory neurons suppress neutrophil and gammadelta $T$ cell responses in bacterial lung infections and lethal pneumonia. Nat Med 24, 417-426, doi:10.1038/nm.4501 (2018).

Pinho-Ribeiro, F. A. et al. Blocking Neuronal Signaling to Immune Cells Treats Streptococcal Invasive Infection. Cell 173, 1083-1097 e1022, doi:10.1016/j.cell.2018.04.006 (2018).

Yissachar, N. et al. An Intestinal Organ Culture System Uncovers a Role for the Nervous System in Microbe-Immune Crosstalk. Cell 168, 1135-1148 e1112, doi:10.1016/j.cell.2017.02.009 (2017).

Chiu, I. M. et al. Bacteria activate sensory neurons that modulate pain and inflammation. Nature 501, 52-57, doi:10.1038/nature12479 (2013).

Ding, W., Stohl, L. L., Wagner, J. A. \& Granstein, R. D. Calcitonin gene-related peptide biases Langerhans cells toward Th2-type immunity. J Immunol 181, 6020-6026, doi:10.4049/jimmunol.181.9.6020 (2008). Boilly, B., Faulkner, S., Jobling, P. \& Hondermarck, H. Zhao, C. M. et al. Denervation suppresses doi:10.1126/scitranslmed.3009569 (2014). Kashem, S. W. et al. Nociceptive Sensory Fibers Drive Interleukin-23 Production from CD301b+ Dermal Dendritic Cells and Drive Protective Cutaneous Immunity. Immunity 43, 515-526, doi:10.1016/j.immuni.2015.08.016 (2015). model. Pigment Cell Melanoma Res 30, 428-435, doi:10.1111/pcmr.12591 (2017).

Meeth, K., Wang, J. X., Micevic, G., Damsky, W. \& Bosenberg, M. W. The YUMM lines: a series of congenic mouse melanoma cell lines with defined genetic alterations. Pigment Cell Melanoma Res 29, 590-597, doi:10.1111/pcmr.12498 (2016).

Cohen, J. A. et al. Cutaneous TRPV1(+) Neurons Trigger Protective Innate Type 17 Anticipatory Immunity. Cell 178, 919-932 e914, doi:10.1016/j.cell.2019.06.022 (2019).

Michoud, F. et al. Epineural optogenetic activation of

Talbot, S. et al. Vagal sensory neurons drive mucous cell metaplasia. J Allergy Clin Immunol 145, 1693-1696 e1694, doi:10.1016/j.jaci.2020.01.003 (2020).

Pellett, S., Tepp, W. H., Whitemarsh, R. C., Bradshaw, M. \& Johnson, E. A. In vivo onset and duration of action varies for botulinum neurotoxin A subtypes 1-5. Toxicon 107, 37-42, doi:10.1016/j.toxicon.2015.06.021 (2015). Binshtok, A. M., Bean, B. P. \& Woolf, C. J. Inhibition of nociceptors by TR
channel blockers. Nature 449, 607-610, doi:10.1038/nature06191 (2007). Jerby-Arnon, L. et al. A Cancer Cell Program Promotes T Cell Exclusion and Resistance to Checkpoint Blockade. Cell 175, 984-997 e924, doi:10.1016/j.cell.2018.09.006 (2018).

Tirosh, I. et al. Dissecting the multicellular ecosystem of metastatic melanoma by single-cell RNA-seq. Science $\mathbf{3 5 2}$, 189-196, doi:10.1126/science.aad0501 (2016).

Haqq, C. et al. The gene expression signatures of melanoma progression. Proc Natl Acad Sci U S A 102, 6092-6097, doi:10.1073/pnas.0501564102 (2005). Harlin, H. et al. Chemokine expression in melanoma metastases associated with CD8+ T-cell recruitment. Cancer Res 69, 3077-3085, doi:10.1158/0008-5472.CAN-08-2281 (2009).

48 Riker, A. I. et al. The gene expression profiles of primary and metastatic melanoma yields a transition point of tumor progression and metastasis. BMC Med Genomics 1, 13, doi:10.1186/1755-8794-1-13 (2008).

49 Talantov, D. et al. Novel genes associated with malignant melanoma but not benign melanocytic lesions. Clin Cancer Res 11, 7234-7242, doi:10.1158/1078-0432.CCR-05-0683 (2005). Larkin, J. et al. Five-Year Survival with Combined Nivolumab and Ipilimumab in Advanced Melanoma. N Engl J Med 381, 1535-1546, doi:10.1056/NEJMoa1910836 (2019).

51 Long, G. V. et al. Nivolumab for Patients With Advanced Melanoma Treated Beyond Progression: Analysis of 2 Phase 3 Clinical Trials. JAMA Oncol 3, 1511-1519, doi:10.1001/jamaoncol.2017.1588 (2017). Wolchok, J. D. et al. Overall Survival with Combined Nivolumab and Ipilimumab in Advanced Melanoma. N Engl J Med 377, 1345-1356, doi:10.1056/NEJMoa1709684 (2017). Global Expression Profiling. Cell Rep 22, 1301-1312, doi:10.1016/j.celrep.2018.01.006 (2018). doi:10.1016/j.jaci.2020.12.644 (2021).

55 Foster, S. L., Seehus, C. R., Woolf, C. J. \& Talbot, S. Sense and Immunity: Context-Dependent Neuro-Immune Interplay. Front Immunol 8, 1463, doi:10.3389/fimmu.2017.01463 (2017). 
Lai, N. Y. et al. Gut-Innervating Nociceptor Neurons Regulate Peyer's Patch Microfold Cells and SFB Levels to Mediate Salmonella Host Defense. Cell 180, 33-49 e22, doi:10.1016/j.cell.2019.11.014 (2020).

57 Solinski, H. J. et al. Nppb Neurons Are Sensors of Mast Cell-Induced Itch. Cell Rep 26, $3561-3573$ e3564, doi:10.1016/j.celrep.2019.02.089 (2019).

58 Trankner, D., Hahne, N., Sugino, K., Hoon, M. A. \& Zuker, C. Population of sensory neurons essential for asthmatic hyperreactivity of inflamed airways. Proc Natl Acad Sci U S A 111, 11515-11520, doi:10.1073/pnas.1411032111 (2014).

59 Chiu, I. M. et al. Transcriptional profiling at whole population and single cell levels reveals somatosensory neuron molecular diversity. Elife 3, doi:10.7554/eLife.04660 (2014).

60 Oetjen, L. K. et al. Sensory Neurons Co-opt Classical Immune Signaling Pathways to Mediate Chronic Itch. Cell 171, 217-228 e213, doi:10.1016/j.cell.2017.08.006 (2017).

61 Riol-Blanco, L. et al. Nociceptive sensory neurons drive interleukin-23-mediated psoriasiform skin inflammation. Nature 510, 157-161, doi:10.1038/nature13199 (2014).

62 Cancer Genome Atlas Research, N. et al. The Cancer Genome Atlas Pan-Cancer analysis project. Nat Genet 45, 1113-1120, doi:10.1038/ng.2764 (2013).

63 Heng, T. S., Painter, M. W. \& Immunological Genome Project, C. The Immunological Genome Project: networks of gene expression in immune cells. Nat Immuno/ 9, 1091-1094, doi:10.1038/ni1008-1091 (2008).

64 Crosson, T. et al. Profiling of how nociceptor neurons detect danger-new and old foes. Journal of internal medicine 286, 268-289 (2019).

65 Ray, P. et al. Comparative transcriptome profiling of the human and mouse dorsal root ganglia: an RNA-seq-based resource for pain and sensory neuroscience research. Pain 159, 1325 (2018).

66 Chiu, I. M. et al. Transcriptional profiling at whole population and single cell levels reveals somatosensory neuron molecular diversity. Elife 3, e04660 (2014).

67 Usoskin, D. et al. Unbiased classification of sensory neuron types by large-scale single-cell RNA sequencing. Nature neuroscience 18, 145-153 (2015).

$68 \mathrm{Li}, \mathrm{C}$., Wang, S., Chen, Y. \& Zhang, X. Somatosensory neuron typing with high-coverage single-cell RNA sequencing and functional analysis. Neuroscience bulletin 34, 200-207 (2018).

69 Goswami, S. C. et al. Molecular signatures of mouse TRPV1-lineage neurons revealed by RNA-Seq transcriptome analysis. The Journal of Pain 15, 1338-1359 (2014).

70 Kupari, J., Häring, M., Agirre, E., Castelo-Branco, G. \& Ernfors, P. An atlas of vagal sensory neurons and their molecular specialization. Cell reports 27, 2508-2523. e2504 (2019).

71 Castle, J. C. et al. Exploiting the mutanome for tumor vaccination. Cancer Res 72, 1081-1091, doi:10.1158/00085472.CAN-11-3722 (2012).

72 Monaco, G. et al. RNA-Seq Signatures Normalized by mRNA Abundance Allow Absolute Deconvolution of Human Immune Cell Types. Cell Rep 26, 1627-1640 e1627, doi:10.1016/j.celrep.2019.01.041 (2019). 


\section{Supplementary methods}

Patients' biopsy. Melanoma biopsies were collected at Sanford Health and classified by a board-certified pathologist. Given that all patient samples were de-identified. Sanford Health IRB company established that these samples were not considered human subjects research, and no IRB number was assigned. DERM103 patient sample classified as a malignant melanoma from the left posterior shoulder, MART-1 and HMB45 positive, melanocytic in nature (Elastic positive), and MIB-1 positive (increased proliferation). DERM107 patient sample classified as malignant melanoma, high mitotic index, invasive, stage pT2a, MART-1, SOX-10, P16, and HMB45 positive. DERM110 patient sample classified as malignant melanoma; high mitotic index, Clark's level IV, stage pT1b; the tumor appeared to be invading the epidermis with a fusion of malignant sheets of cells, strongly positive for SOX-10, MART-1, HMB45 with a moderate increase in proliferative index.

IHC Staining and Scoring. Patient melanoma skin biopsies were stained using a BenchMark XT slide staining system (Ventana Medical Systems, Inc.). The Ventana iView DAB detection kit was used as the chromogen, and slides were counterstained with hematoxylin and anti-TRPV1 (Alomone Labs, ACC-030; 1:100). H\&E staining followed standard procedures. TRPV1 IHC stained human samples were analyzed on an Olympus BX51 bright-field microscope. Sections were viewed under $20 \times$ magnification. Five random fields per sample for both tumor and adjacent normal tissue were analyzed and scored on a scale from 0-3. Scores were averaged. A score of 0 indicates no appreciated nerve fibres in the evaluated field; ${ }^{+1}$ indicates sparse nerve fibres; ${ }^{+} 2$ indicates $5-20$ nerve fibres; ${ }^{+} 3$ indicates $>20$ nerve fibres.

Animals. The Institutional Animal Care and Use Committees of Boston Children's Hospital and the Université de Montréal (CDEA: \#19027; \#19028) approved all animal procedures. Mice were housed in standard environmental conditions (12h light/dark cycle; $23^{\circ} \mathrm{C}$; food and water ad libitum) at facilities accredited by the Association for Assessment and Accreditation of Laboratory Animal Care.

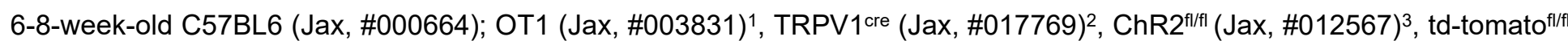
$(\mathrm{Jax}, \# 007908)^{4}$, DTA ${ }^{\mathrm{fl} / \mathrm{fl}}(\mathrm{Jax}, \# 009669)^{5}$, and QuASR2 $2^{\mathrm{fl} / \mathrm{fl}}(\mathrm{Jax}, \# 028678)^{6}$ mice were purchased from Jackson Laboratory. NaV1.8 $8^{\text {cre }}$ mice ${ }^{7}$ were generously supplied by Professor Rohini Kuner (Heidelberg University) and John Wood (UCL).

We used the cre/lox toolbox to engineer the various mice lines used (TRPV1 ${ }^{\text {cre::DTA }}$ fl/wt, TRPV1 $^{\text {cre: }}$ QuASR2fl/wt,

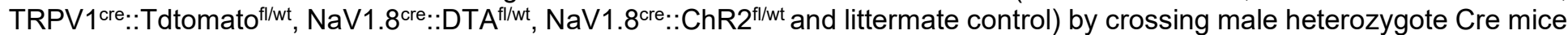
with female homozygous loxP mice. All Cre driver lines used were viable and fertile, and abnormal phenotypes were not detected. Offspring were tail-clipped; tissue was used to assess the presence of transgene by standard PCR, as described by Jackson Laboratory. Offspring were used at 8 weeks of age.

Cell lines. B16F0 ${ }^{8}$ (ATCC, \#CRL-6322), B16F10 ${ }^{9}$ (ATCC, \#CRL-6475), B16F10-OVA ${ }^{10}$ (Matthew F. Krummel, UCSF), B16F10-OVA-mCherry ${ }^{11}$ (Matthew F. Krummel, UCSF), B16F10-eGFP, YUMM1.7 ${ }^{12}$ (Marcus Bosenberg, Yale U), and nontumorigenic keratinocytes (CellnTEC, \#MPEK-BL6100) were cultured in complete Dulbecco's Modified Eagle's Medium high glucose (DMEM, Corning, \#10-013-CV) supplemented with $10 \%$ fetal bovine serum FBS, Seradigm, \#3100) and $1 \%$ penicillin/streptomycin (Corning, \#MT-3001-Cl), and maintained at $37^{\circ} \mathrm{C}$ in a humidified incubator with $5 \% \mathrm{CO}_{2}$. YUMMER1.7 13 (Marcus Bosenberg, Yale) cells were cultured in DMEM F12 (Gibco; \#11320033) supplemented with 10\% fetal bovine serum (Seradigm, \#3100), 1\% penicillin/streptomycin (Corning, \#MT-3001-Cl) and MEM nonessential amino acids (Corning; \#25$025 \mathrm{Cl}$ ), and maintained at $37^{\circ} \mathrm{C}$ in a humidified incubator with $5 \% \mathrm{CO}_{2}$.

Cancer inoculation and volume measurement. Cancer cells ( $1 \times 10^{5}$ to $5 \times 10^{5}$ cells) were resuspended in Phosphate Buffered Saline (PBS, Corning \#21040CV) and injected to the mice's skin right flank (i.d., $150 \mu \mathrm{l}$ ) or hindpaw (i.d., $20 \mu \mathrm{l}$ ). Growth was assessed daily using a handheld digital caliper and tumor volume was determined by the formula $\left(\mathrm{L} \times \mathrm{W}^{2} \times 0.52\right){ }^{14}$, in which $L=$ length and $W=$ width .

Survival analysis. In dedicated groups of mice, orthotopic B16F10-mCherry-OVA (5x105 cells; i.d.) cells were administered to intact and nociceptor ablated mice and survival was measured until day 22 and determined by tumor reaching a volume $\geq 1000$ $\mathrm{mm}^{3}$ or pre-determined ethical endpoint (i.e, ulceration). In B16F10-mCherry-OVA inoculated mice treated with QX-314 or BIBN4096, the survival was measured until day 19 and determined by tumor reaching a volume $\geq 800 \mathrm{~mm}^{3}$ or pre-determined ethical endpoint.

iDISCO imaging. Whole-mount immunohistochemistry of tumors was performed using an iDISCO protocol with methanol pretreatment optimized for tumors ${ }^{24}$. Briefly, adult animals (8 weeks) were perfused with $25 \mathrm{~mL}$ of PBS (HyClone) and $25 \mathrm{~mL}$ of $4 \%$ paraformaldehyde (Sigma) sequentially at room temperature (RT). Tumors were postfixed with $4 \%$ PFA for $6 \mathrm{~h}$ at $4{ }^{\circ} \mathrm{C}$. For methanol pre-treatment, fixed tumors were washed sequentially in $50 \%$ methanol (in PBS) for $1 \mathrm{hr}, 100 \%$ methanol for $1 \mathrm{hr}$, and then bleached in $5 \% \mathrm{H}_{2} \mathrm{O}_{2}$ in $20 \% \mathrm{DMSO} /$ methanol overnight at $4{ }^{\circ} \mathrm{C}$. Tumors were subsequently rehydrated in $100 \%$ methanol for $1 \mathrm{hr}$ twice, $20 \%$ DMSO/methanol for $1 \mathrm{hr}$ twice, $50 \%$ methanol in PBS for $1 \mathrm{hr}$, PBS for $1 \mathrm{hr}$ twice, and PBS/0.2\% Triton X-100 for $1 \mathrm{hr}$ twice at RT. Tumors were then left in PBS/0.2\% Triton X-100/20\% DMSO/0.3 M glycine (Sigma) overnight at RT and blocked in PBS/0.2\% Triton X-100/10\% DMSO/6\% donkey serum (Jackson ImmunoResearch)/anti-CD16/CD32 (Fc block; Bio X cell) overnight at RT. Tumors were subsequently washed in PBS/0.2\% Tween-20/10 mg/mL heparin (Sigma) 
(PTwH), for $1 \mathrm{~h}$ twice at RT before incubation with antibody mix (GFP (Aves Labs) at 1:500, mCherry (OriGene) at 1:500, biotinconjugated $\mathrm{CD}^{+}$antibodies (BioLegend; 1:50) in $\mathrm{PTwH} / 5 \%$ DMSO/3\% donkey serum/Fc block 1:100 for 4 days at RT. Tumors were extensively washed in PTwH at least 6 times over the course of 1 day at RT. Tumors were further incubated with a secondary antibody mix, including a panel of species-specific anti-lgG $\left(\mathrm{H}^{+} \mathrm{L}\right)$ Alexa Fluor 488 or 546 -conjugated antibodies (Invitrogen or Jackson ImmunoResearch) and ATTO 647N-conjugated streptavidin (Sigma), all at 1:500, in PTwH/5\% DMSO/3\% donkey serum/Fc block $1: 100$ for 3 more days at RT. Tumors were washed in the same way as after primary antibody incubation for 1 day. Immunolabeled tumors were then processed for clearing, which included sequential incubation with $50 \%$ methanol for $1 \mathrm{hr}$ at RT, 100\% methanol for $1 \mathrm{hr}$ three times at RT, and a mixture of 1 part benzyl alcohol (Sigma): 2 parts benzyl benzoates (Sigma) overnight at $4^{\circ} \mathrm{C}$. For tdTomato and GFP immunolabeling, mCherry and GFP antibodies were preabsorbed against tumors from tdTomato animals overnight at RT prior to use.

Tumor and tdLN digestion. Mice were euthanized when the tumor reached a volume of $800-1500 \mathrm{~mm}^{3} 8,9,15$. Tumors and their draining lymph nodes (tdLN) were harvested. Tumors were enzymatically digested in DMEM $+5 \%$ FBS (Seradigm, \#3100) + $2 \mathrm{mg} / \mathrm{ml}$ collagenase D (Sigma, \#11088866001) + $1 \mathrm{mg} / \mathrm{ml}$ Collagenase IV (Sigma, \# C5138-1G) + $40 \mathrm{ug} / \mathrm{ml}$ DNAse I (Sigma, $\# 10104159001)$ under constant shaking $(40 \mathrm{~min}, 37 \mathrm{oC})$. The cell suspension was centrifuged at $400 \mathrm{~g}$ for $5 \mathrm{~min}$. The pellet was resuspended in $70 \%$ Percoll gradient (GE Healthcare), overlaid with $40 \%$ Percoll, and centrifuged at $500 \mathrm{~g}$ for 20 min at room temperature with acceleration and deceleration at 1 . The cells were aspirated from the Percoll interface and passed through a $70-\mu \mathrm{m}$ cell strainer. Tumor-draining lymph nodes were dissected in PBS $+5 \%$ FBS, mechanically dissociated using a plunger, strained $(70 \mu \mathrm{m})$, and washed with PBS.

Drugs. QX-314 ${ }^{16}$ (Tocris, \#2313; 0.3\%) was injected (i.d.) daily in 5 points around the tumor (treatment began from day 1 following tumor inoculation. BIBN4096 (Tocris, \#4561; $5 \mathrm{mg} / \mathrm{kg}$ ) was injected (i.p.) on days 6, 8, 10, 12, and 14 following

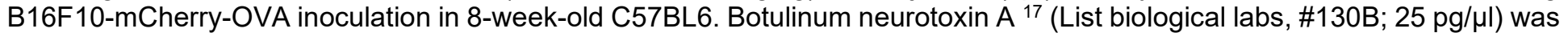
injected (i.d.) three and one days prior to tumor inoculation.

aPDL1 treatment. Orthotopic B16F10-mCherry-OVA cells $(500,000$ cells; i.d.) were inoculated into 8-week-old male and female sensory neuron intact or ablated mice. On days 7, 10, and 13 post-tumor inoculations, the mice were treated with aPDL1 ${ }^{18}$ (Bio X Cell, \#BE0101, 6 mg/kg; i.p.) or its vehicle. Twenty-one days post-tumor inoculation, the impact of $\alpha$ PDL1 was analyzed on tumor growth and TILs exhaustion.

Orthotopic B16F10-mCherry-OVA cells (500,000 cells; i.d.) were inoculated into 8-week-old male and female mice. Starting one day post-inoculation, a group of vehicle-exposed B16F10-bearing mice was injected with QX-314 (0.3\%; i.d., 5 sites around the tumor) once daily. On days 7, 10, and 13 post-tumor inoculations, the mice were exposed to aPD-L1 18 (Bio X Cell, \#BE0101, $6 \mathrm{mg} / \mathrm{kg}$; i.p.) or its vehicle. Sixteen days post-tumor inoculation, the impact of aPDL1 was analyzed on tumor growth and TILs exhaustion.

aPDL1 treatment in mice with same size tumor. Orthotropic B16F10-mCherry-OVA cells (500,000 cells, i.d.) were injected into a cohort of nociceptor neuron-ablated mice 3 days prior to one given to nociceptor intact animals. Mice from each group with similar tumor size $\left(\sim 85 \mathrm{~mm}^{3}\right)$ were selected and exposed to $\alpha$ PD-L1 ${ }^{18}$ (Bio X Cell, \#BE0101, $6 \mathrm{mg} / \mathrm{kg}$; i.p.) once every 3 days for a total of 9 days. The impact of aPDL1 treatment was analyzed on tumor growth until day 20.

Orthotropic B16F10-mCherry-OVA cells (500,000 cells, i.d.) were injected into mice treated with QX-314 (0.3\%; i.d.) 2-3 days prior to being given to vehicle-exposed mice. Mice from each group with similar tumor size $\left(\sim 100 \mathrm{~mm}^{3}\right)$ were selected and exposed to aPD-L1 ${ }^{18}$ (Bio X Cell, \#BE0101, $6 \mathrm{mg} / \mathrm{kg}$; i.p.) once every 3 days for a total of 9 days. The impact of aPDL1 treatment was analyzed on tumor growth until day 18.

Immunophenotyping. Single cells were resuspended in FACS buffer (PBS, 2\% FCS, EDTA), Fc blocked (0.5 mg/ml, $10 \mathrm{~min}$; BD Biosciences, \#553141) and stained (30 min, $4^{\circ} \mathrm{C}$ ) with ZombieAqua (BioLegend, \#423102) and a combination of the following monoclonal antibodies: anti-CD45-BV421 (1:100, BioLegend, \#103134), anti-CD11b-APC/Cy7 (1:100, BioLegend, \#101226), anti-CD8-AF700 (1:100, BioLegend, \#100730), anti-CD4-PerCP/Cyanine5.5 (1:100, BioLegend, \#100540), anti-PD1-PE-Cy7 (1:100, BioLegend, \#109110), anti-Lag3-PE (1:100, BioLegend, \#125208), anti-Tim-3-APC (1:100, BioLegend, \#119706).

Intracellular cytokine staining. Cells were stimulated $(4 \mathrm{~h})$ with a cell activation cocktail and Brefeldin A (BioLegend, \#423303), washed, fixed/permeabilized (BD Biosciences; \#554714) and stained with anti-IFN-y-BV650 (BioLegend, \#505832), anti-TNFa-FITC (BioLegend, \#506304), anti-Granzyme B-APC (BioLegend, \# 396407), and anti-IL2-Pacific Blue (Biolegend, \# 503820).

In vivo depletion of CD3. $200 \mu \mathrm{g} /$ mouse of anti-mouse CD3 (18) (Bio X Cell, \#BE0001-1) was injected (i.p.) 3 days prior to B16F10-OVA-mCherry inoculation $\left(1 \times 10^{5}\right.$; i.d.) and continued every 3 days. Blood samples were taken twice weekly to confirm depletion, and tumor growth was measured daily. 
In vivo. CGRP injection. TRPV1 ablated mice were injected (i.d.) once daily with recombinant CGRP (100nM) at 5 points around the tumor (treatment began once the tumor was visible), and tumor growth was measured daily by a handheld digital caliper. Mice were sacrificed, and tumor-infiltrated $\mathrm{CD} 8^{+}$cells exhaustion was immunophenotyped by flow cytometry.

BM transplant. Total CD8 ${ }^{+}$T-cells were isolated from the spleen of wild-type $\left(C D 45.1^{+}\right)$or RAMP1/- $\left(C D 45.2^{+}\right)$mice, expanded and amplified in vitro using a mouse T-cell Activation/Expansion Kit (Miltenyi cat \#130-093-627). CD8 ${ }^{+}$cells from RAMP1-- and RAMP1 ${ }^{\text {wt }}$ were injected separately or $1: 1 \mathrm{mix}$ through tail vein of Rag $1^{-/}$mice. One week after, the mice were inoculated with B16F10-OVA-mCherry cancer cells $\left(5 \times 10^{5}\right)$, and tumor growth was measured daily using a handheld digital caliper. On day 14 , tumor and draining lymph node were harvested, and RAMP1-- $\left(C D 45.1^{+}\right)$and RAMP1wt $\left(C D 45.1^{+}\right)$CD8 ${ }^{+}$T-cells were immunophenotyped or FACS-purified.

For FACS-purified cells, RAMP1/- and RAMP1wt $C D 8^{+}$T-cells RNA-seq libraries were constructed using Illumina TruSeq Stranded RNA LT Kit (Illumina, San Diego, CA) following the manufacturer's instructions. Illumina sequencing was performed at the Fulgent Genetics (Temple City, CA). Reads were aligned to the Mouse mm10 reference genome using STAR v2.721. Aligned reads were assigned to genic regions using featureCounts function from subread v1.6.4 ${ }^{22}$. Gene expression levels were represented by TPM (Transcripts Per Kilobase Million). Hierarchical clustering was computed using heatmap.2 function (ward.D2 method) from gplots R package. Differential gene expression analysis was carried out by DeSeq2 v1.28.123.

Pain behaviour. $1 \times 10^{5}$ B16F10 cells or $1 \times 10^{5}$ non-cancerous keratinocytes (MPEK-BL6) were inoculated intradermally in the mice's left hind paw. On alternate days, thermal and mechanical sensitivity was evaluated using a plantar test (Hargreaves method) analgesia meter (21) (IITC Life Science, Model 390G) and von Frey monofilaments (21) (Ugo Basile, \#52-37450-275), respectively.

Itch. Orthotopic B16F10-mCherry-OVA cells (500,000 cells; i.d.) were inoculated into 8-week-old male and female sensory neuron intact or ablated mice left flank. Starting one day post-inoculation, QX-314 (0.3\%; i.d., 5 sites around the tumor) or its vehicle was administered once daily to a group of nociceptor littermate control mice. 12-14 days post tumor inoculation, $\sim 30 \%$ of nociceptor intact and vehicle-exposed B16F10-injected mice showed spontaneous itch. Itch was recorded for 30min twice weekly (9:00-11:00am) and manually scored. Results of day 21 was reported as itch bouts/30min.

Skin explant. 3h post-exposure to vehicle $(100 \mu \mathrm{l})$, QX-314 $(0.3 \%, 100 \mu \mathrm{l})$ or BoNT/A $(25 \mathrm{pg} / \mu \mathrm{L}, 100 \mu \mathrm{l})$, tumor-surrounding skin was harvested using $10 \mathrm{~mm}$ punch biopsies. The biopsies were transferred into 24-well plates and cultured in DMEM containing $1 \mu \mathrm{l} / \mathrm{ml}$ of protease inhibitor (Sigma, \#P1860) and capsaicin (1 $\mu \mathrm{M}$. Sigma, \#M2028). After $30 \mathrm{~min}$ incubation (37 $\mathrm{C})$, the supernatant was collected and CGRP release analyzed using a commercial ELISA ${ }^{17}$ (Cayman Chemical, \#589001).

Neuron culture. Mice were sacrificed, and dorsal root ganglia (DRG) were dissected out into DMEM medium (Corning, \#10013-CV), completed with $50 \mathrm{U} / \mathrm{mL}$ penicillin and $50 \mu \mathrm{g} / \mathrm{ml}$ streptomycin (Corning, \#MT-3001-Cl) and 10\% FBS (Seradigm, \#3100). Cells were then dissociated in HEPES buffered saline (Sigma, \#51558) completed with $1 \mathrm{mg} / \mathrm{mL}$ collagenase IV (Sigma, \#C0130) + $2.4 \mathrm{U} / \mathrm{mL}$ Dispase II (Sigma, \#04942078001) and incubated for $80 \mathrm{~min}$ at $37^{\circ} \mathrm{C}$. Ganglia were triturated with glass Pasteur pipettes of decreasing size in supplemented DMEM medium, then centrifuged over a $10 \%$ BSA gradient and plated on laminin (Sigma, \#L2020) coated cell culture dishes. Cells were cultured with Neurobasal-A medium (Gibco, \#21103049) completed with $0.05 \mathrm{ng} / \mu \mathrm{L}$ NGF (Life Technologies, \#13257-019), $0.002 \mathrm{ng} / \mu \mathrm{L}$ GDNF (PeproTech, \#450-51-10), 0.01 mM AraC (Sigma, \#C6645) and 200 mM L-Glutamine (VWR, \#02-0131) (19), and B-27 supplement (Gibco, \#17504044).

Calcium imaging. L3-L5 DRG neurons were harvested and co-cultured with B16F10, B16F0, or MPEK-BL6 for 24-48h. The cells were then loaded with $5 \mathrm{mM}$ Fura-2 AM (BioVision, \#2243) in complete Neurobasal-A medium for 30 min at $37^{\circ} \mathrm{C}$, washed in Standard Extracellular Solution (SES, $145 \mathrm{mM} \mathrm{NaCl}, 5 \mathrm{mM} \mathrm{KCl}, 2 \mathrm{mM} \mathrm{CaCl} 2,1 \mathrm{mM} \mathrm{MgCl} 2,10 \mathrm{mM}$ glucose, $10 \mathrm{mM} \mathrm{HEPES}$, $\mathrm{pH}$ 7.5), and response to noxious ligands (100 nM capsaicin; $100 \mu \mathrm{M}$ AITC; $1 \mu \mathrm{M}$ ATP) was analyzed at room temperature. Ligands were flowed (15s) directly onto neurons using perfusion barrels followed by buffer washout (105-sec minimum) (19). Cells were illuminated by a UV light source (Xenon lamp, 75 watts, Nikon), $340 \mathrm{~nm}$ and $380 \mathrm{~nm}$ excitation alternated by an LEP MAC 5000 filter wheel (Spectra services), and fluorescence emission was captured by Cool SNAP ES camera (Princeton Instruments). 340/380 ratiometric images were processed, background corrected and analyzed (IPLab software; Scientific Analytics), and Microsoft Excel used for post hoc analyses. For SLPI, the DRG neurons were cultured for 24h and then loaded with $5 \mathrm{mM}$ Fura-2 AM in complete Neurobasal-A medium for $45 \mathrm{~min}$ at $37^{\circ} \mathrm{C}$, washed into SES, and response to noxious ligands (0-10 ng/ml of mouse recombinant SLPI (LifeSpan BioSciences, \#LS-G13637-10); $1 \mu \mathrm{M}$ capsaicin; $50 \mathrm{mM} \mathrm{KCl)} \mathrm{were} \mathrm{analyzed}$ at room temperature.

Immunofluorescence. $2 \times 10^{3}$ DRG neurons were co-cultured with $2 \times 10^{4} \mathrm{~B} 16 \mathrm{~F} 10-\mathrm{mCherry}-\mathrm{OVA}$ for $24-48 \mathrm{~h}$. The cells were fixed (4\% paraformaldehyde; $30 \mathrm{~min}$ ), permeabilized (0.1\% Triton X-100, $20 \mathrm{~min}$ ), and blocked (PBS, 0.1\% Triton X-100, 5\% $\mathrm{BSA}, 30 \mathrm{~min}$ ). The cells were rinsed (PBS), stained, and mounted with vectashield containing DAPI (Vector Laboratories, \#H1000). Images were acquired using a Ti2 Nikon fluorescent microscope.

Neurite length, ramification index, and intersecting radii. TRPV1+ nociceptors (TRPV1 ${ }^{\text {cre: }:: T d-t o m a t o}{ }^{\mathrm{f} / \mathrm{wt}}$ ) were cultured alone $\left(2 \times 10^{3}\right.$ cells) or co-cultured $\left(2 \times 10^{4}\right.$ cells) with B16F10-GFP, B16F0, or non-tumorigenic keratinocytes (MPEK-BL6). After 
48h, cells were fixed (see immunofluorescence section), and images were acquired using a Ti2 Nikon fluorescent microscope. TRPV $1^{+}$(td-tomato) neurons' neurite length was measured using a neurite tracer macro in Image $\mathrm{J}$ developed by the Fournier $\mathrm{lab}^{19}$, while the Schoenen ramification index (SRI) and the number of intersecting radii were measured by a Sholl analysis ${ }^{20}$ macro in ImageJ.

CD8 $^{+}$T cell isolation. 6-8-week-old male and female mice were euthanized, spleen harvested in ice-cold PBS (5\% FBS), and mechanically dissociated. The cells were strained $(70 \mu \mathrm{m})$, RBC lysed (Life Technologies, \#A1049201; 2 min), and counted using a hemocytometer. Total CD8 ${ }^{+}$T-cells were magnet sorted (Stem cell, \#19853A) and cultured (DMEM + FBS 10\%, Pen/Strep 1\% + non-essential amino acid (Corning, \#25-025-Cl) + vitamin + $\beta$-mercaptoethanol (Gibco, \#21985-023) + LGlutamine (VWR, \#02-0131) + sodium pyruvate (Corning, \#25-000-Cl)). Cell purity was systematically confirmed after magnet sorting and the numbers of $\mathrm{CD} 8{ }^{+} \mathrm{CD} 62 \mathrm{~L}^{\text {hi }}$ immunophenotyped by flow cytometry. To generate cytotoxic $\mathrm{T}$ lymphocytes, $2 \times 10^{5}$ $\mathrm{CD}^{+} \mathrm{T}$-cells were seeded and stimulated for $48 \mathrm{~h}$ under $\mathrm{T}_{\mathrm{c} 1}$ inflammatory condition $(2 \mathrm{\mu g} / \mathrm{ml}$ plate bounded $\mathrm{aCD} 3 / \mathrm{aCD} 28$ (Bio X Cell, \#BE00011, \#BE00151) + $10 \mathrm{ng} / \mathrm{ml} \mathrm{rlL}-12$ (BioLegend, \#577008) + $10 \mu \mathrm{g} / \mathrm{ml}$ of anti-IL-4 (Bio X Cell, \#BE0045).

In vitro cytotoxic CD8 ${ }^{+} \mathrm{T}$-cell stimulation with neuron-conditioned media. Naive or ablated DRG neurons were cultured (72h) in Neurobasal-A medium supplemented with $0.05 \mathrm{ng} / \mu \mathrm{L}$ NGF (Life Technologies, \#13257-019) and $0.002 \mathrm{ng} / \mu \mathrm{L}$ GDNF (PeproTech, \#450-51-10). After 48h, the neurobasal medium was removed, neurons were washed with PBS, and $200 \mathrm{ul} / \mathrm{well}$ of T-cell media supplemented with $1 \mu \mathrm{l} / \mathrm{ml}$ peptidase inhibitor (Sigma, \#P1860) and, in certain cases, capsaicin $(1 \mu \mathrm{M})$ or $\mathrm{KCl}$ $(50 \mathrm{mM})$ was added to DRG neurons. The conditioned media or vehicle were collected after $30 \mathrm{~min}$ and added to Tc1 CD8 $8^{+} \mathrm{T}-$ cells for another $96 \mathrm{~h}$. The CD8+ T-cells expression of exhaustion markers (PD-1, Lag-3 and Tim-3) and cytokine (IFN- - , TNF$\alpha$ and IL-2) were analyzed by flow cytometry.

$\mathrm{CD}^{+} \mathrm{T}$-cells were isolated and stimulated under $\mathrm{T}_{\mathrm{c} 1}$ condition in 96 wells plate. After $48 \mathrm{~h}$, cells were treated with either CGRP $(0.1 \mu \mathrm{M})$ or PBS in the presence of peptidase inhibitor $(1 \mu \mathrm{M})$ for another $96 \mathrm{~h}$. Expression of PD-1, Lag-3, and Tim-3, as well as IFN- $\gamma$, TNF- $\alpha$, and IL-2, was immunophenotyped by flow cytometry.

CD8 $^{+}$T-cell and DRG neurons co-culture. Naive DRG neurons $\left(2 \times 10^{4}\right)$ were seeded in a 96 -well-plate with T-cell media (supplemented with $0.05 \mathrm{ng} / \mu \mathrm{L}$ NGF (Life Technologies, \#13257-019), $0.002 \mathrm{ng} / \mu \mathrm{L}$ GDNF (PeproTech, \#450-51-10). One day after, $\mathrm{T}_{\mathrm{c} 1} \mathrm{CD}^{+}$cells $\left(1 \times 10^{5}\right)$ were added to the neurons in the presence of IL-2 (BioLegend, \#575408). In some instances, cocultures were stimulated with either capsaicin $(1 \mu \mathrm{M})$ or $\mathrm{KCl}(50 \mathrm{mM})$. After $96 \mathrm{~h}$, the cells were collected by centrifugation $(5$ min at $1300 \mathrm{rpm})$, stained, and immunophenotyped by flow cytometry.

CD8+ T-cell and DRG neurons co-culture RNA sequencing and data processing. $1 \times 10^{4}$ naive TRPV1 ${ }^{\text {Cre }:: Q u A S R 2 / / w t} D R G$ neurons were co-cultured with $1 \times 10^{5} \mathrm{~B} 16 \mathrm{~F} 10-\mathrm{mCherry}-\mathrm{OVA}$ overnight in T-cell media (supplemented with $0.05 \mathrm{ng} / \mu \mathrm{L} \mathrm{NGF}$ (Life Technologies, \#13257-019), $0.002 \mathrm{ng} / \mu \mathrm{L}$ GDNF (PeproTech, \#450-51-10). After 48h, the cells were detached and TRPV1 neurons (CD45- eGFP ${ }^{+}$), and OVA-specific CD8 ${ }^{+}$T-cells (eGFP- $\mathrm{CD}^{-} 5^{+} \mathrm{CD}^{+} \mathrm{CD}^{+}$) were FACS-purified and cell supernatant was collected for ELISAs. RNA-seq libraries were constructed using Illumina TruSeq Stranded RNA LT Kit (Illumina, San Diego, $\mathrm{CA}$ ) following the manufacturer's instructions. Illumina sequencing was performed at the Fulgent Genetics (Temple City, CA). Reads were aligned to the Mouse mm10 reference genome using STAR v2.721. Aligned reads were assigned to genic regions using featureCounts function from subread v1.6.4 22. Gene expression levels were represented by TPM (Transcripts Per Kilobase Million). Hierarchical clustering was computed using heatmap.2 function (ward.D2 method) from gplots R package. Differential gene expression analysis was carried out by DeSeq2 v1.28.123.

ELISA on CD8 ${ }^{+}$T-cell and DRG neurons co-culture. SLPI (R\&D Systems, \#DY1735-05), TSLP (BioLegend, \# 434104), and HMGB1 (Novus Biologicals, \#NBP2-62767) levels were measured in the cells' supernatant (as described above) using commercial ELISA.

In vitro DRG neuron silencing with Botox. Naive DRG neurons $\left(2 \times 10^{4}\right)$ were seeded in a 96 well plate with neurobasal medium supplemented with NGF and GDNF. Neurons were pretreated with $50 \mathrm{pg} / \mathrm{ml}$ of BoNT/A for $24 \mathrm{~h}$. After 24 hours, the culture medium was removed, neurons were washed with PBS and $200 \mu \mathrm{l} /$ well of T-cell media supplemented with $1 \mu \mathrm{l} / \mathrm{ml}$ peptidase inhibitor, and $\mathrm{KCl}(40 \mathrm{mM})$ was added to DRG neurons. The conditioned media or vehicle were collected after 30 min and added to Tc1 CD8 ${ }^{+}$T-cells for another $96 \mathrm{~h}$.

In vitro RAMP1 blockade. aCGRP (Bioss, \#bs-0791R), CGRP 8 -37 (Tocris, \#1169), and BIBN4096 (Tocris, \#4561) were used in culture. CD8+ T-cells were treated with BIBN4096 $(4 \mu \mathrm{M}) 6 \mathrm{~h}$ before being exposed to the neurons' conditioned media. For CGRP antagonists, the neurons' conditioned media were incubated for $1 \mathrm{~h}$ with $2 \mu \mathrm{g} / \mathrm{ml}$ of $\alpha C G R P$ and $1 \mu \mathrm{g} / \mathrm{ml}$ of CGRP $8-37$ before being added to the $\mathrm{CD} 8^{+} \mathrm{T}$-cells.

In vitro B16F10 cells apoptosis. $2 \times 10^{4}$ naive TRPV1Cre::QuASR2 ${ }^{\text {fl/wt }}$ DRG neurons were co-cultured with $1 \times 10^{5}$ B16F10mCherry-OVA overnight in T-cell media (supplemented with $0.05 \mathrm{ng} / \mu \mathrm{L}$ NGF (Life Technologies, \#13257-019), 0.002 ng/ $/ \mathrm{L}$ GDNF (PeproTech, \#450-51-10). One day after, $4 \times 10^{5}$ stimulated OVA-specific CD8 ${ }^{+} \mathrm{T}$-cells under $\mathrm{T}_{\mathrm{c} 1}$ condition were added to the co-culture. After 48h, the cells were detached by trypsin (Gibco, \#2062476) and collected by centrifugation (5 min at $1300 \mathrm{rpm}$ ), stained using anti-Annexin V, 7-AAD (BioLegend, \#640930), and anti-CD8 for 20 minutes at $4^{\circ} \mathrm{C}$, and were 
immunophenotyped by flow cytometry. Alternatively, co-cultures were imaged every $6 \mathrm{~h}$ using a fluorescent microscope (Nikon, $\mathrm{Ti} 2$ ) and cell proliferation was measured.

For neuron condition media, $4 \times 10^{5}$ stimulated OVA-specific CD8 ${ }^{+}$T-cells were added to $1 \times 10^{5}$ B16F10-mCherry-OVA and treated with collected fresh condition media. After $48 \mathrm{~h}$, cells were stained as mentioned. For CGRP and RAMP1 blocker, $4 \times 10^{5}$ stimulated OVA-specific CD8 ${ }^{+}$T-cells were added to $1 \times 10^{5}$ B16F10-mCherry-OVA, and cells were stained after $24 \mathrm{~h}$.

Triple co-culture RNA sequencing and data processing. $1 \times 10^{4}$ naive TRPV1Cre::QuASR2 $2^{\mathrm{f} / \mathrm{wt}} \mathrm{DRG}$ neurons were co-cultured with $1 \times 10^{5}$ B16F10-mCherry-OVA overnight in T-cell media (supplemented with $0.05 \mathrm{ng} / \mu \mathrm{L}$ NGF (Life Technologies, \#13257019), $0.002 \mathrm{ng} / \mu \mathrm{L}$ GDNF (PeproTech, \#450-51-10). One day after, $4 \times 10^{5}$ stimulated OVA-specific CD8 ${ }^{+}$T-cells under Tc1 condition were added to the co-culture. After 48h, the cells were detached and TRPV1 neurons (CD45- eGFP+ $\mathrm{mCherry}^{-}$), B16F10-mCherry-OVA (CD45- eGFP- mCherry ${ }^{+}$), and OVA-specific CD8 ${ }^{+}$T-cells (eGFP- mCherry- CD45 ${ }^{+} \mathrm{CD}^{+} \mathrm{CD}^{+}$) were FACS-purified. RNA-seq libraries were constructed using Illumina TruSeq Stranded RNA LT Kit (Illumina, San Diego, CA) following the manufacturer's instructions. Illumina sequencing was performed at the Fulgent Genetics (Temple City, CA). Reads were aligned to the Mouse mm10 reference genome using STAR v2. ${ }^{21}$. Aligned reads were assigned to genic regions using featureCounts function from subread v1.6.4 ${ }^{22}$. Gene expression levels were represented by TPM (Transcripts Per Kilobase Million). Hierarchical clustering was computed using heatmap.2 function (ward.D2 method) from gplots R package. Differential gene expression analysis was carried out by DeSeq2 v1.28.123.

B16F10 survival. 1 x $10^{5}$ B16F10 cells were cultured in 6-well-plate and challenged with BoNT/A (0-50 pg/ $\left.\mathrm{\mu l}\right)$ for $24 \mathrm{~h}, \mathrm{QX}-314$ $(0-1 \%)$ for $72 \mathrm{~h}$, BIBN4096 (1-4 $\mu \mathrm{M})$ for $24 \mathrm{~h}$ or their vehicle. B16F10 cell survival was assessed using anti-annexin $V$ staining and measured by flow cytometry or counted using a hemocytometer.

In-silico analysis of neuronal expression profile using RNA Sequencing and Microarray datasets. Publicly available RNA Gene expression from seven datasets ${ }^{25-30}$ were downloaded from the NCBI GEO portal. RNA Gene expression values of genes of interest were extracted. Expression values from single cell sequencing were averaged for all cells. To be able to compare expression from datasets that were generated using different techniques (sc-RNAseq, bulk RNAseq, microarrays) and normalization methods (TPM, RPKM, RMA, UMI), all genes of interest were ratioed over Trpv1 expression, then multiplied by 100 , and the $\log _{10}$ of these values were plotted as a heatmap. Kupari ${ }^{30}$ used single-cell RNA-sequencing of JNC neurons while Usoskin ${ }^{28}$ and $\mathrm{Li}^{29}$ used single-cell RNA-sequencing of lumbar neurons. Chiu ${ }^{26}$ respectively measured gene expression by microarrays of whole and FACS-sorted Nav1.8+ lumbar neurons. Goswami ${ }^{25}$ performed RNA-sequencing of TRPV $1^{+}$lumbar neurons, whilst Ray ${ }^{27}$ performed RNA-sequencing of human lumbar neurons.

In-silico analysis of melanoma patients' tumor expression profile using single-cell RNA sequencing. Using the publicly available Broad Institute single-cell portal, we proceed to an in-silico analysis of single-cell RNA-sequencing of human melanoma biopsies. We assessed the gene profile of RAMP1-expressing and RAMP1-negative T cell in patients' tumor with metastatic melanoma ${ }^{31}$. Similarly, we assessed the genetic program of RAMP1-expressing and RAMP1-negative CD8 ${ }^{+} \mathrm{T}^{-c e l l s}$ in patient with melanoma ${ }^{32}$. The latter dataset was also used to analyze the genetic profile of $\mathrm{CD} 8^{+} \mathrm{T}$-cells in patients responsive to immune checkpoint blocker or unresponsive to such treatment as well as the genetic profile of malignant melanoma cells (defined as CD90-CD45-) from 10 different patients' biopsies ${ }^{32}$. Individual cell data are shown as a $\log _{2}$ of $1+$ (transcript per million / 10). Experimental details and cell clustering were defined in Tirosh et al., ${ }^{31}$ and Jerby-Arnon et al., ${ }^{32}$.

In-silico analysis of human immunocytes expression profile. Publicly available RNA Gene expression from Monaco et al., ${ }^{33}$ was downloaded from the NCBI GEO portal. Read counts normalized to transcripts per million protein-coding genes (pTPM) values for genes of interest were extracted. Expression values from single cell sequencing were averaged for all cells. Experimental details and cell clustering were defined in Monaco et al., ${ }^{33}$.

In-silico analysis of cultured B16F10 cells expression profile. Publicly available RNA Gene expression from Castle et al., ${ }^{34}$ was downloaded from the NCBI GEO portal. Read counts normalized to transcript per million (TPM) for genes of interest were extracted. Experimental details and cell clustering were defined in Castle et al., ${ }^{34}$.

In-silico analysis of mouse immunocytes expression profile using the Immgen database. Using the publicly available Immgen database we proceed to an in-silico analysis of RNA-sequencing data (DESeq2 data) of various mouse immunocytes. As per Immgen protocol, RNA-sequencing reads were aligned to the mouse genome GENCODE GRCm38/mm10 primary assembly and gene annotations VM16 with STAR 2.5.4a. The ribosomal RNA gene annotations were removed from general transfer format file. The gene-level quantification was calculated by featureCounts. Raw reads count tables were normalized by median of ratios method with DESeq2 package from Bioconductor and then converted to GCT and CLS format. Samples with less than 1 million uniquely mapped reads were automatically excluded from normalization. Experimental details were defined in https://www.immgen.org/Protocols/lmmGenULI RNAseq methods.pdf.

In-silico analysis of melanoma patient biopsy expression profile using BULK microarray sequencing. As described in Haqq et al., samples from forty-five cutaneous melanomas and 18 benign melanocytic skin nevus biopsies $(\sim 5-20 \mu m)$ were 
harvested, amplified and transcriptome profiled using Affymetrix U133A microarrays. Data were downloaded from oncomine database (www.oncomine.org) as $\log _{2}$ (median centred intensity) and gene of interest were displayed as heatmaps. Experimental details and cell clustering were defined in Haqq et al., ${ }^{35}$.

Melanoma patient Kaplan Meier survival analysis. OncoLnc (www.oncolnc.org) contains survival data for 8,647 patients from 21 cancer studies performed by The Cancer Genome Atlas (TCGA) ${ }^{36}$. Using OncoLnc we assess transcript expression of a user-defined list of 333 neuronal-enriched genes (neuronal membrane proteins, neural stem cell markers, transcription factors, ion channel receptors, and neuropeptides) in 459 skin cancer (SKCM) tumor biopsies from the Cancer Genome Atlas (TCGA) database. Of these genes, 206 were expressed, and 108 were selected based on their negative Cox coefficient value, indicating a link between lower gene expression and improved patient survival. Kaplan-Meier curves show the patients' survival after segregation in two groups defined by their low or high expression of a gene of interest. Details of patients can be found in the Cancer Genome Atlas (TCGA) ${ }^{36}$ and computational analysis were defined by Anaya et al., 2016 at https://doi.org/10.7717/peeri-cs.67.

Statistics. Data expressed as mean \pm S.E.M. Statistical significance determined by Mantel-Cox regression (survival curve), one-way or two-way ANOVA for multiple comparisons and two-tail unpaired Student t-test for single variable comparison. Pvalues less than 0.05 were considered significant. Numbers of animals are defined in figures. 


\section{Supplementary method references.}

$1 \quad$ Hogquist, K. A. et al. T cell receptor antagonist peptides induce positive selection. Cell 76, 17-27, doi:10.1016/00928674(94)90169-4 (1994).

2 Crosson, T. et al. FcepsilonR1 expressing nociceptors trigger allergic airway inflammation. J Allergy Clin Immunol, doi:10.1016/j.jaci.2020.12.644 (2021).

3 Madisen, L. et al. A toolbox of Cre-dependent optogenetic transgenic mice for light-induced activation and silencing. Nat Neurosci 15, 793-802, doi:10.1038/nn.3078 (2012).

4 Madisen, L. et al. A robust and high-throughput Cre reporting and characterization system for the whole mouse brain. Nat Neurosci 13, 133-140, doi:10.1038/nn.2467 (2010).

$5 \quad$ Voehringer, D., Liang, H. E. \& Locksley, R. M. Homeostasis and effector function of lymphopenia-induced "memorylike" T cells in constitutively T cell-depleted mice. J Immunol 180, 4742-4753, doi:10.4049/jimmunol.180.7.4742 (2008). Lou, S. et al. Genetically Targeted All-Optical Electrophysiology with a Transgenic Cre-Dependent Optopatch Mouse. J Neurosci 36, 11059-11073, doi:10.1523/JNEUROSCI.1582-16.2016 (2016). Agarwal, N., Offermanns, S. \& Kuner, R. Conditional gene deletion in primary nociceptive neurons of trigeminal ganglia and dorsal root ganglia. Genesis 38, 122-129, doi:10.1002/gene.20010 (2004).

$8 \quad$ Fidler, I. J. Biological behavior of malignant melanoma cells correlated to their survival in vivo. Cancer Res 35, 218224 (1975).

$9 \quad$ Fidler, I. J. \& Kripke, M. L. Metastasis results from preexisting variant cells within a malignant tumor. Science 197, 893895, doi:10.1126/science.887927 (1977).

10 Moore, M. W., Carbone, F. R. \& Bevan, M. J. Introduction of soluble protein into the class I pathway of antigen processing and presentation. Cell 54, 777-785, doi:10.1016/s0092-8674(88)91043-4 (1988).

Headley, M. B. et al. Visualization of immediate immune responses to pioneer metastatic cells in the lung. Nature 531, 513-517, doi:10.1038/nature16985 (2016).

Meeth, K., Wang, J. X., Micevic, G., Damsky, W. \& Bosenberg, M. W. The YUMM lines: a series of congenic mouse melanoma cell lines with defined genetic alterations. Pigment Cell Melanoma Res 29, 590-597, doi:10.1111/pcmr.12498 (2016).

model. Pigment Cell Melanoma Res 30, 428-435, doi:10.1111/pcmr.12591 (2017).

Twyman-Saint Victor, C. et al. Radiation and dual checkpoint blockade activate non-redundant immune mechanisms in cancer. Nature 520, 373-377, doi:10.1038/nature14292 (2015).

Broz, M. L. et al. Dissecting the Tumor Myeloid Compartment Reveals Rare Activating Antigen-Presenting Cells Critical for T Cell Immunity. Cancer Cell 26, 938, doi:10.1016/j.ccell.2014.11.010 (2014). channel blockers. Nature 449, 607-610, doi:10.1038/nature06191 (2007).

Baral, P. et al. Nociceptor sensory neurons suppress neutrophil and gammadelta $\mathrm{T}$ cell responses in bacterial lung infections and lethal pneumonia. Nat Med 24, 417-426, doi:10.1038/nm.4501 (2018).

Stathopoulou, C. et al. PD-1 Inhibitory Receptor Downregulates Asparaginyl Endopeptidase and Maintains Foxp3 Transcription Factor Stability in Induced Regulatory $\mathrm{T}$ Cells. Immunity 49, 247-263 e247, doi:10.1016/j.immuni.2018.05.006 (2018). Pool, M., Thiemann, J., Bar-Or, A. \& Fournier, A. E. NeuriteTracer: a novel ImageJ plugin for automated quantification of neurite outgrowth. J Neurosci Methods 168, 134-139, doi:10.1016/j.jneumeth.2007.08.029 (2008). Garcia-Segura, L. M. \& Perez-Marquez, J. A new mathematical function to evaluate neuronal morphology using the Sholl analysis. J Neurosci Methods 226, 103-109, doi:10.1016/j.jneumeth.2014.01.016 (2014). (2013). Liao, Y., Smyth, G. K. \& Shi, W. featureCounts: an efficient general purpose program for assigning sequence reads to genomic features. Bioinformatics 30, 923-930, doi:10.1093/bioinformatics/btt656 (2014). Love, M. I., Huber, W. \& Anders, S. Moderated estimation of fold change and dispersion for RNA-seq data with DESeq2. Genome Biol 15, 550, doi:10.1186/s13059-014-0550-8 (2014).

Renier, N. et al. iDISCO: a simple, rapid method to immunolabel large tissue samples for volume imaging. Cell 159, 896-910, doi:10.1016/j.cell.2014.10.010 (2014). analysis. J Pain 15, 1338-1359, doi:10.1016/j.jpain.2014.09.010 (2014).

Chiu, I. M. et al. Transcriptional profiling at whole population and single cell levels reveals somatosensory neuron molecular diversity. Elife 3, doi:10.7554/eLife.04660 (2014).

Ray, P. et al. Comparative transcriptome profiling of the human and mouse dorsal root ganglia: an RNA-seq-based resource for pain and sensory neuroscience research. Pain 159, 1325-1345, doi:10.1097/j.pain.0000000000001217 (2018). 
29 Li, C., Wang, S., Chen, Y. \& Zhang, X. Somatosensory Neuron Typing with High-Coverage Single-Cell RNA Sequencing and Functional Analysis. Neurosci Bull 34, 200-207, doi:10.1007/s12264-017-0147-9 (2018).

30 Kupari, J., Haring, M., Agirre, E., Castelo-Branco, G. \& Ernfors, P. An Atlas of Vagal Sensory Neurons and Their Molecular Specialization. Cell Rep 27, 2508-2523 e2504, doi:10.1016/j.celrep.2019.04.096 (2019).

31 Tirosh, I. et al. Dissecting the multicellular ecosystem of metastatic melanoma by single-cell RNA-seq. Science $\mathbf{3 5 2}$, 189-196, doi:10.1126/science.aad0501 (2016).

32 Jerby-Arnon, L. et al. A Cancer Cell Program Promotes T Cell Exclusion and Resistance to Checkpoint Blockade. Cell 175, 984-997 e924, doi:10.1016/j.cell.2018.09.006 (2018).

33 Monaco, G. et al. RNA-Seq Signatures Normalized by mRNA Abundance Allow Absolute Deconvolution of Human Immune Cell Types. Cell Rep 26, 1627-1640 e1627, doi:10.1016/j.celrep.2019.01.041 (2019).

34 Castle, J. C. et al. Exploiting the mutanome for tumor vaccination. Cancer Res 72, 1081-1091, doi:10.1158/00085472.CAN-11-3722 (2012).

35 Haqq, C. et al. The gene expression signatures of melanoma progression. Proc Natl Acad Sci U S A 102, 6092-6097, doi:10.1073/pnas.0501564102 (2005).

36 Cancer Genome Atlas Research, N. et al. The Cancer Genome Atlas Pan-Cancer analysis project. Nat Genet 45, 1113-1120, doi:10.1038/ng.2764 (2013). 